\title{
Abstracts from the 27th Annual International Atrial Fibrillation Symposium January 13-15, 2022
}

E. Heist ${ }^{1}$

${ }^{1}$ no affiliation

February 2, 2022

Abstracts from the 27th Annual International Atrial Fibrillation Symposium January 13-15, 2022

Late-Breaking Clinical Trials and First Report Clinical Investigations: AFS 2022 -01 to -07

AFS2022-01

Abstract Title: Clinical Outcomes of Lattice-Tip Focal Ablation for Atrial Fibrillation: Toggling Between Pulsed Field and Radiofrequency Energy

Author(s): Vivek Reddy, MD

Co-Author(s):

petr Peichl, MD PhD

Elad Anter, MD

Gediminas Rackauskas, MD PhD

Jan Petru, MD PhD

Moritoshi Funasako, MD

Kentaro Minami, MD

Jacob Koruth, MD

Andrea Natale, MD

Hiroshi Nakagawa, MD

Pierre Jais, MD

Germanas Marinskis, MD PhD

Audrius Aidietis, MD PhD

Josef Kautzner, MD PhD

Petr Neuzil, MD PhD

MOUNT SINAI MEDICAL CENTER

vivek reddy one gustave levy place, box 1030 mount sinai hospital

Introduction | Objectives: 
In first-in-human trials (NCT04141007, NCT04194307), a lattice-tip catheter using focal RF or PF (pulsed field) energy proved able to treat PAF or PerAF with a PVI strategy of either RFA anteriorly / PFA posteriorly $(\mathrm{RF} / \mathrm{PF})$, or $\mathrm{PFA}$ throughout $(\mathrm{PF} / \mathrm{PF})$ with good lesion durability. Herein, we assessed the long-term clinical outcomes of safety and efficacy.

\section{Methods:}

The $8 \mathrm{Fr}$ lattice catheter has a compressible $9 \mathrm{~mm}$ nitinol tip, and is used with a custom mapping system and RF \& PF generators (Affera Inc, MA). Toggling between RF \& PF, point-by-point PVI was performed using biphasic PFA (2-5 sec; $1.3-2.0 \mathrm{kV}[ \pm]$ ) posteriorly, and either temp-controlled irrigated $\mathrm{RFA}\left(\mathrm{T}_{\max } 73^{\circ} \mathrm{C} ; 5\right.$ sec) or PFA anteriorly. Linear lesions were placed with PFA and/or RFA. Protocol-driven remapping was at $\sim 3$ mo post-index ablation. Based on remap data, the PF waveform/delivery evolved: PULSE1 (2-5s, 76 pts), PULSE2 (4s, 47 pts) and the final optimized PULSE3 (4s, 55 pts).

\section{Results:}

A total of 178 pts ( 3 centers-14 operators, age 59.7 \pm 9.4 yrs; $\mathrm{M} / \mathrm{F}=128 / 50 ; \mathrm{PAF} / \mathrm{PerAF}=70 / 108)$ underwent PVI with $\mathrm{RF} / \mathrm{PF}$ (79 pts) or PF/PF (99 pts). The PVI duration time (transpired from $1^{\text {st }}$ to last lesion) was $21.3 \pm 5.8$ min. Linear lesions included 78 mitral, 121 CTI and 130 LA roof lines (including 38 box lesions), with transpired ablation times of $3.9 \pm 2.2,2.0 \pm 1.4$ and $1.9 \pm 1.7 \mathrm{~min} / \mathrm{pt}$, respectively. All lesion sets (100\%) were acutely successful. Fluoroscopy time was $4.3 \pm 3.1 \mathrm{~min}$. At $96 \pm 43$ days, remapping was performed on 122 pts (69\%). Per-PV / per-patient durability rates were 51\%/32\%,87\%/64\% and $97 \%$ / $90 \%$ for PULSE1, PULSE2 and PULSE3, respectively. After $325 \pm 67$ days follow-up, the 1-yr KM estimates for freedom from AT/AF/AFL for the full cohort were $78.3 \pm 5.0 \%$ and $76.8 \pm 4.4 \%$ for PAF and PerAF, respectively; for PULSE3 cohort alone, the outcome for PerAF was $84.5 \pm 5.1 \%$ at 308 days. There was 1 primary adverse event - inflammatory pericardial effusion not requiring intervention. There was no esophageal fistula, stroke/TIA, phrenic injury, PV stenosis, or other late safety events.

\section{Conclusions:}

AF ablation using the lattice-tip RF/PF catheter reveals excellent clinical outcomes, providing strong rationale for the recently commenced FDA randomized trial.

\section{AFS2022-01}

\section{Uploaded File(s)}

\section{Lattice-Tip RF/PF Ablation Catheter: 1-Year Clinical Effectiveness}

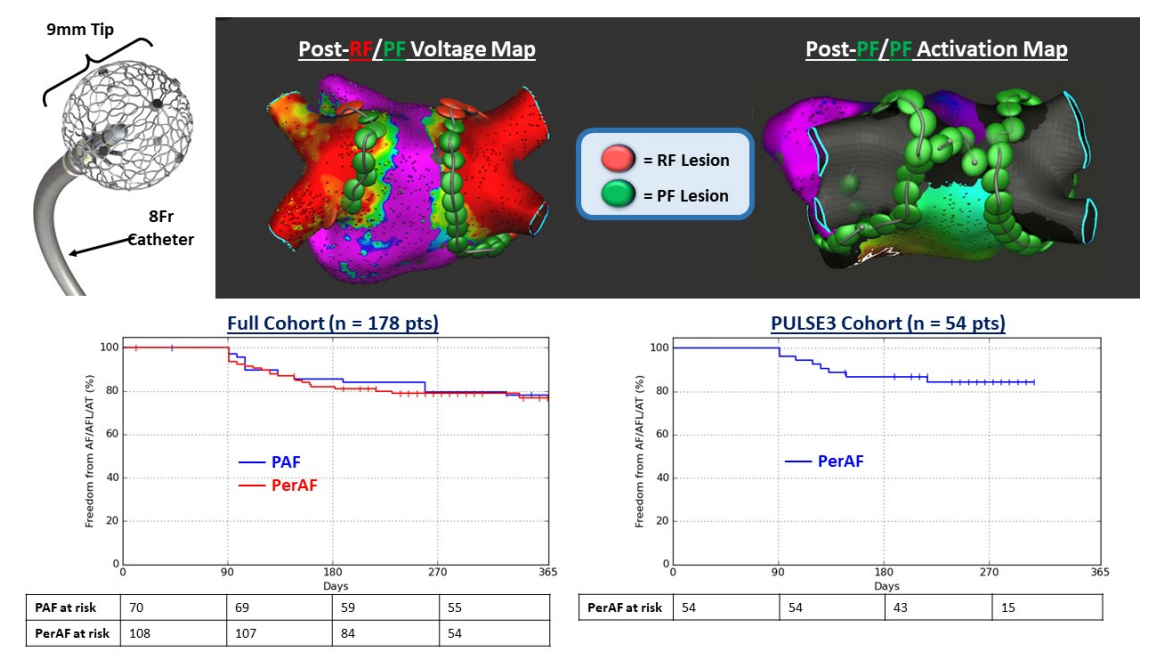




\section{AFS2022-02}

Abstract Title: 12M Safety/Effectiveness Of Very High Power Short Duration Pulmonary Vein Isolation With A Contact Force-Sensing, Temperature-Controlled Radiofrequency Catheter: The Prospective, Nonrandomized, Multicenter Q-FFICIENCY Trial

Author(s): Jose Osorio, MD

Co-Author(s):

Ayman Hussein, MD - Cleveland Clinic Foundation

M. Craig Delaughter, MD - Texas Health Heart \& Vascular

George Monir, MD - AdvantHealth Orlando

Andrea Natale, MD - Texas Cardiac Arrhythmia Institute

Srinivas Dukkipati, MD - Mount Sinai School of Medicine

Saumil Oza, MD - St Vincent's Medical Center

Emile Daoud, MD - Ohio State University Medical Center

Luigi Di Biase, MD - Montefiore Medical Center at Albert Einstein College of Medicine

Moussa Mansour, MD - Massachusetts General

Robert Fishel, MD - JFK Medical Center

Miguel Valderrabano, MD - Houston Methodist Research Institute

Kenneth Ellenbogen, MD - Virginia Commonwealth University

Grandview Medical Center Alabama Cardiovascular Group

3686 Grandview Pkwy \#720 Birmingham, AL 35243

Introduction | Objectives: A novel contact force (CF) sensing catheter was optimized for temperaturecontrolled radiofrequency $(\mathrm{RF})$ ablation. It has 3 microelectrodes and 6 thermocouples allowing power modulation to maintain a target temperature and the delivery of very high-power, short-duration (vHPSD; $90 \mathrm{~W}, 4 \mathrm{~s}) \mathrm{RF}$. We evaluated the 12-month (M) safety and effectiveness of the catheter for paroxysmal atrial fibrillation (PAF) ablation, primarily with vHPSD mode in combination with conventional power (CP; 25-50 W) mode, as needed.

Methods: In this US multicenter (22 sites), nonrandomized investigational study, patients underwent pulmonary vein $(\mathrm{PV})$ isolation with vHPSD as primary ablation mode; CP was used for PV touch-up or non-PV ablation. Primary safety endpoint was incidence of primary adverse events (PAE) [?]7 days postprocedure. Primary effectiveness was freedom from documented atrial tachyarrhythmia recurrence and additional predefined failure modes (acute failure, repeat ablation, new/higher dose AAD).

Results: Of 191 participants (63.5 \pm 10.7 years, $\mathrm{CHADS}_{2}-\mathrm{VASc} 2.4 \pm 1.5,60.7 \%$ male), 166 were ablated with the investigational catheter. PVI with vHPSD-only was achieve in 89/164 participants with confirmed entrance block for all PVs. Median (Q1/Q3) procedure time (including 20-minute waiting time) and RF application time were $132(108 / 171)$ and 9.8 (6.8/15.7) minutes, respectively. Mean number of RF applications was $121.2 \pm 43.7$, most using vHPSD (median of 93.3\%). Mean maximum temperature, impedance drop, and $\mathrm{CF}$ per RF application were similar between vHPSD and $\mathrm{CP}$ modes $\left(45.1 \pm 5.3\right.$ vs $44.5 \pm 3.9{ }^{\circ} \mathrm{C}$; $9.8 \pm 8.5$ vs $9.1 \pm 3.7 \Omega \cdot 15.5 \pm 9.7$ vs $15.2 \pm 8.9 \mathrm{~g}$, respectively). PAE rate was $3.6 \%$, with no reports of deviceor procedure-related death, atrioesophageal fistula, stroke, transient ischemic attack, or severe PV stenosis. Kaplan-Meier estimated $12 \mathrm{M}$ primary effectiveness and freedom from repeat ablation during the evaluation period were $76.7 \%$ and $92.1 \%$, respectively. 
Conclusions: Temperature-controlled PAF ablation with vHPSD, alone or in combination with CP, is effective and highly efficient without compromising safety.

\section{AFS2022-03}

Abstract Title: Impact Of High-frequency Low-tidal-volume Mechanical Ventilation On First-pass Pulmonary Vein Isolation And Procedural Times During Radiofrequency Ablation Of Atrial Fibrillation: A Multicenter Experience

Author(s): Jose Osorio, MD, FHRS

Co-Author(s):

Allyson Varley, PhD - Heart Rhythm Clinical and Research Solutions

Chris Thorne, PhD - Heart Rhythm Clinical and Research Solutions

Gustavo Morales, MD - Arrhythmia Institute at Grandview

Anil Rajendra, MD, FHRS - Arrhythmia Institute at Grandview

Gunther Schrappe, MBA - Heart Rhythm Clinical and Research Solutions

Joshua Silverstein, MD

Paul Zei, MD, PhD, FHRS

Jorge Romero, MD, FHRS

Arrhythmia Institute at Grandview

3686 Grandview Pkwy \#720, Birmingham, AL 35243, United States

Introduction | Objectives: Catheter stability is crucial for predictable energy delivery to the myocardium and the resultant creation of durable lesions during radiofrequency (RF) ablation of atrial fibrillation (AF). Brief periods of apnea and JET ventilation can improve catheter stability but have limitations and potential complications. High-frequency, low-tidal-volume (HFLTV) ventilation was shown in single center studies to be a simpler and safer alternative with similar benefits. We evaluated the impact of HFLTV during RF AF ablation on procedural short-term efficacy, efficiency, and safety in a large multicenter study.

Methods: 1,052 patients from 25 institutions were prospectively enrolled in a multicenter RF AF ablation registry (REAL-AF) from January 2018 to November 2021. Conventional ventilation only was used up until April 2020 [standard ventilation (SV) group] when HFLTV was implemented progressively at different centers (30 breaths/min and tidal volume 200ml). High-power, short-duration ablation with 40-50 watts was used in all cases, and other ablation parameters remained unchanged. Procedural characteristics and incidence of severe anesthesia and procedural relate adverse events (AE) were compared.

Results: Patients in the HFLTV group $(\mathrm{n}=413)$ had significantly shorter procedure time when compared to SV group $(\mathrm{n}=639)(77.5 \pm 38.7 \mathrm{~min}$ vs $103.3 \pm 46.5 \mathrm{~min}, \mathrm{p}<0.001)$. Similarly, total and pulmonary vein RF times were shorter in the HFLTV group when compared to the SV group (16.44 \pm 8.3 min vs. $23.58 \pm 10.3$ min, $\mathrm{p}<0.001$ and $12.42 \pm 5$ min vs $18.2 \pm 6.9 \mathrm{~min}, \mathrm{p}<0.001$, respectively). First-pass pulmonary vein (PV) isolation rates were higher in the HFLTV group [( $84 \%$ vs. $80 \%, \mathrm{p}=0.02)$ (left PVs: $90 \%$ vs. $84 \%, \mathrm{p}=0.01$ and right PVs: $78 \%$ vs. $75 \%, \mathrm{p}=0.37)]$. AEs at 30-day follow-up were similar.

Conclusions: HFLTV mechanical ventilation during RF AF ablation was associated with a significantly higher first-pass PV isolation rate, and shorter total procedural and pulmonary vein RF times without an increase in complications. Further studies are indicated to better understand the impact of HFLTV on catheter stability and long-term outcomes in AF ablation.

\section{AFS2022-03}




\section{Uploaded File(s)}

High Frequency Low Tidal Volume Mechanical Ventilation During RF Atrial Fibrillation Ablation

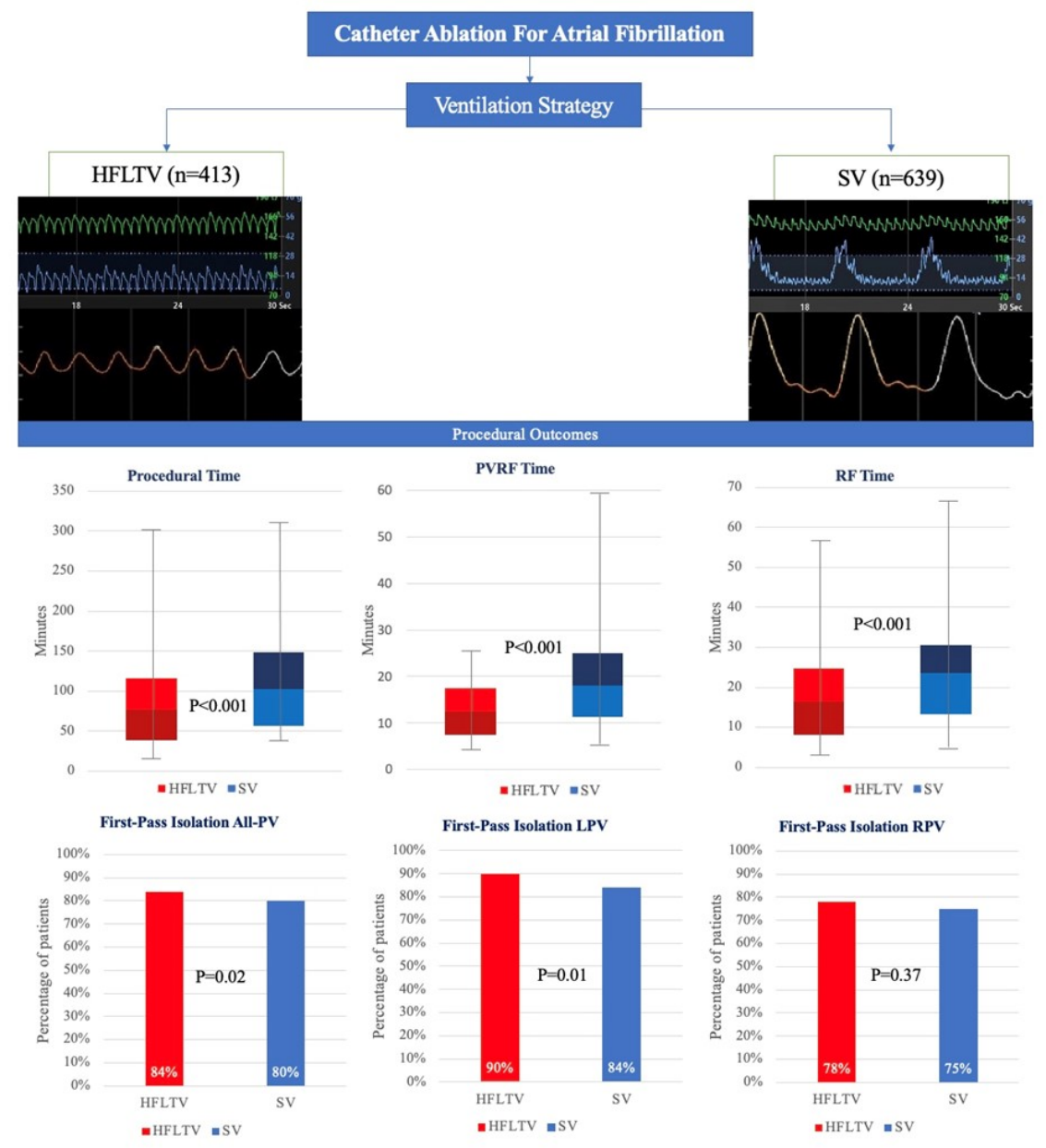

\section{AFS2022-04}

Abstract Title: First in Human Experience and Acute Procedural Outcomes using a Novel Pulsed Field Ablation System: The PULSED AF Pilot Trial

Author(s): Atul Verma, MD

\section{Co-Author(s):}

Lucas Boersma, MD - St Antonius Hospital, Amsterdam, Netherlands

David Haines, MD - Beaumont Hospital, Royal Oak, USA

Andrea Natale, MD - St David's Hospital, Austin, USA

Francis Marchlinski, MD - Hospital of the University of Pennsylvania, Philadelphia, USA

Prashanthan Sanders, MBBS - Royal Adelaide Hospital, Adelaide, Australia 
Hugh Calkins, MD - Johns Hopkins Hospital, Baltimore, USA

Douglas Packer, MD - Mayo Clinic, Rochester, USA

John Hummel, MD - OSU Wexner Medical Center, Columbus, USA

Birce Onal, PhD - Medtronic

Sofi Rosen, PhD - Medtronic

Karl-Heinz Kuck, MD - University Heart Center Lübeck, Lübeck, Germany

Gerhard Hindricks, MD - The Heart Center Leipzig, Leipzig, Germany

Bradley Wilsmore, MD - John Hunter Hospital, Newcastle, Australia

Southlake Regional Health Centre

596 Davis Dr, Newmarket, ON L3Y 2P9, Canada

Introduction | Objectives: Pulsed field ablation (PFA) is a novel form of ablation using electrical fields to ablate cardiac tissue. There is limited data assessing the feasibility and safety of this type of ablation in humans. This is a first-in-human extended pilot phase study describing the usage of PFA for atrial fibrillation (AF) ablation.

Methods: PULSED AF (NCT04198701) is a non-randomized, prospective, multi-center, global, pre-market clinical study. This first-in-human pilot phase evaluated the feasibility, acute safety and efficacy of pulmonary vein isolation (PVI) using a novel PFA system (PulseSelect ${ }^{\mathrm{TM}}$; Medtronic, Inc). The system delivers bipolar, biphasic electrical fields through a circular multi-electrode array catheter. Thirty-eight patients with paroxysmal or persistent, drug-refractory AF undergoing first-time ablation were treated in six centers in Australia, Canada, the United States, and the Netherlands. The primary outcomes were ability to achieve acute PVI intra-procedurally and safety at 30 days.

Results: Of 38 patients, 35 (92\%) had paroxysmal AF with a mean age of $62 \pm 11$ years and a left atrial (LA) diameter of $37 \pm 6 \mathrm{~mm}$. Acute electrical PVI $(\mathrm{n}=152)$ was achieved in all 38 patients. Skin-to-skin procedure time was $160 \pm 91 \mathrm{~min}$, LA dwell time was $82 \pm 35 \mathrm{~min}$, including a required 20-minute waiting period after PVI. Fluoroscopy time was $28 \pm 9 \mathrm{~min}$. The maximum esophageal temperature recorded was $36.1 \pm 0.3^{\circ} \mathrm{C}$, with a mean change of $0.06^{\circ} \mathrm{C}$ from baseline $(\mathrm{n}=7)$. No serious adverse events related to the PFA system occurred in the 30-day follow-up including phrenic nerve injury, esophageal injury, stroke or death.

Conclusions: In this first-in-human extended pilot study, 100\% PVI was achieved using only PFA with no PFA system-related serious adverse events. Data will be published simultaneously to the AF Symposium and Circulation AE.

\section{AFS2022-04}

\section{Uploaded File(s)}

Pulsed Field Ablation catheter (left) and two post-ablation voltage maps (middle, right).
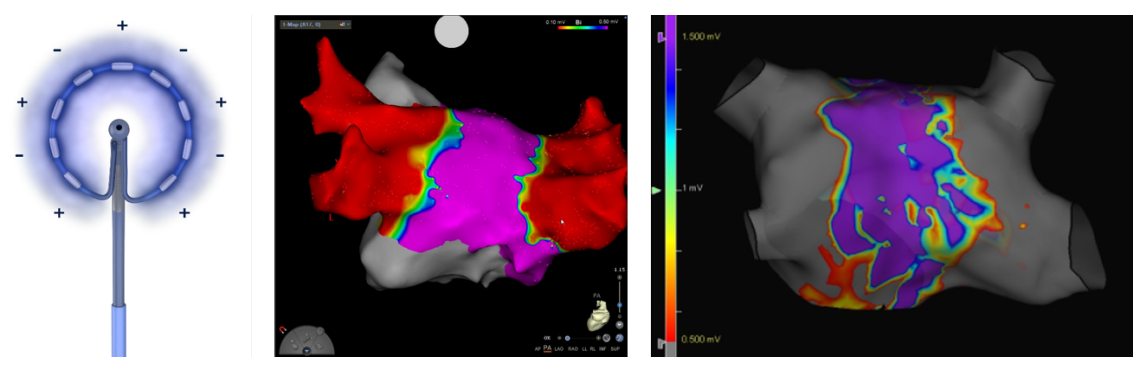


\section{AFS2022-05}

Abstract Title: Safety and Feasibility of Home-Care Neuromodulation and Monitoring Wearable Device for Treatment of Atrial Fibrillation

Author(s): Avi Sabag, MD

Co-Author(s):

Roy Beinart, MD

Eran Leshem, MD

Avi Epstein, MD

Amos Ziv, MSc

Tsahi Holland, MSc

Division of Cardiology, Davidai Arrhythmia Center, Sheba Medical Center

Sheba Medical Center Hospital- Tel Hashomer, Derch sheba 2, Ramta Gan, 52662, IL

Introduction | Objectives: Autonomic modulation is an evolving field in arrhythmia management. tVNS Vagus nerve stimulation suppressed atrial fibrillation (AF) in humans. Median nerve neuromodulation was shown to reduce ventricular and atrial arrhythmias in pre-clinical models. We report the feasibility and safety of CardiaCare RR2, a wristband wearable device with neuromodulation and single lead ECG monitoring capabilities.

Methods: Patients arriving to the emergency room (ER) with symptomatic AF were recruited, underwent pharmacological or electrical cardioversion, received standard of care medication, and received a 24-hour ECG Holter monitor. They returned the next day for a supervised in-hospital first neuromodulation session and received the device and phone app for 8 weeks of home use. Patients were asked to conduct two neuromodulation sessions a week and three 30 sec ECG monitoring sessions daily.

Results: Eleven patients were recruited from the ER (age $66 \pm 12$ yrs, $64 \%$ male). One dropped out prior to protocol initiation, ten completed the protocol. Usability rates were 94.7\% for ECG monitoring (1675 recorded and analyzed) and $96.8 \%$ for neuromodulation (155 treatments, Avg 15.5 per patient). No Adverse Events were observed during the supervised in-hospital treatments or the follow up period. No unscheduled emergency department re-visits occurred. One patient reported minimal itching during use of the device, one patient reported feeling more tired, one patient had non-device related bradycardia and a patient with high AF burden received an ablation at week 6. A total of 289 ECG's were taken immediately before and immediately after neuromodulation. In 7 instances, multiple PACs $(13.7 \pm 12.7 \mathrm{PACs} / 2 \mathrm{~min})$ were observed. In all these cases we observed an acute reduction in the PAC burden following the neuromodulation $(5.7 \pm 10 / 2$ min, $58 \%$ reduction; $\mathrm{p}=0.0005)$. Two (20\%) patients had AF recurrences during the 8-week follow-up period.

Conclusions: The use of CardiaCare neuromodulation and monitoring system is safe. Home-use compliance and usability in a real-world AF population is high. Acute PAC reductions and low AF recurrence rates show compelling early indications of possible AF burden reduction with use of the device.

\section{AFS2022-05}

\section{Uploaded File(s)}

Acute PAC reduction and 8 weeks AF reccurence 


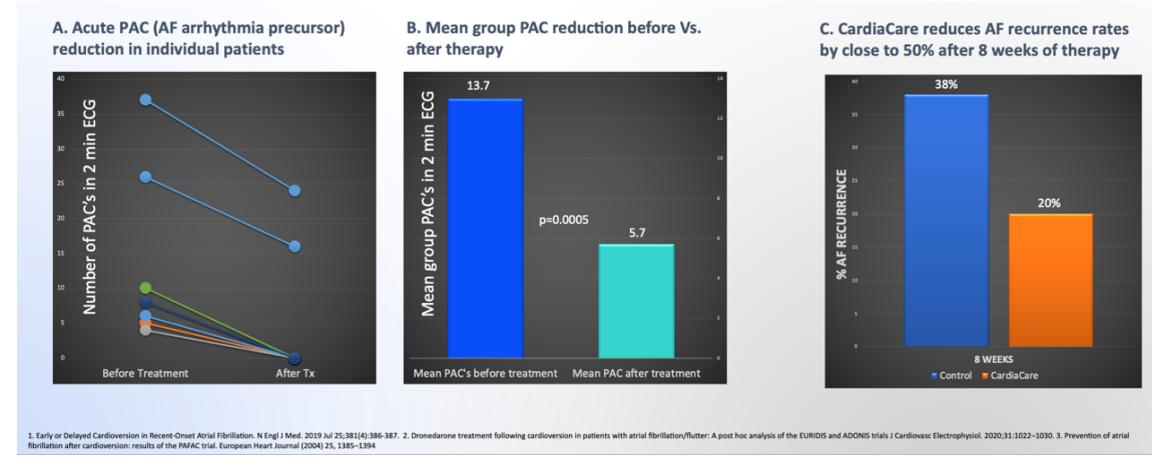

\section{AFS2022-06}

Abstract Title: First-in-Human Clinical Experience of a "Single-Shot" Map-and-Ablate Multielectrode Spherical Array Pulsed Field Ablation Catheter to Isolate Pulmonary Veins

Author(s): Vivek Reddy, MD

\section{Co-Author(s):}

Jacob Koruth, MD

Jan Petru, MD PhD

Moritoshi Funasako, MD

Pavel Hala, MD

Jan Skoda, MD

Milan Chovanec, MD

Lucie Sediva, md

Petr Neuzil, MD PhD

\section{MOUNT SINAI MEDICAL CENTER}

vivek reddy one gustave levy place, box 1030 mount sinai hospital

\section{Introduction | Objectives:}

Most "single-shot" pulsed field ablation (PFA) catheter technologies are either not linked to electroanatomical mapping systems, require serial re-positioning or cannot easily accommodate varying pulmonary vein (PV) anatomical sizes / shapes. Recently, a multielectrode spherical array catheter (Globe, Kardium Inc, Canada) capable of single-shot "mapping and ablation", previously capable of radiofrequency (RF) ablation, has now been enhanced to also deliver PFA. In a first-in-human clinical trial, we assessed the acute safety and efficacy of PV isolation (PVI) using this spherical array PFA catheter.

\section{Methods:}

In a single center single-arm trial of paroxysmal AF ablation, after informed consent and under general anesthesia, ICE-guided transseptal puncture was performed. Through a custom 19 Fr deflectable sheath, the multielectrode spherical array PFA catheter (Globe; Kardium Inc, Canada) was advanced into the left atrium and deployed to its full spherical shape. Using the custom mapping system (GPS; Kardium Inc), the PFA catheter rendered anatomic maps with ostial tags, using contact maps based on blood flow detection. The PFA catheter was positioned at each PV ostium for PVI (1.6-2 kV/application; typically ungated - 3 
sec. PV entrance and exit block were assessed. Post-procedure endoscopy (EGD) and brain MRI occurred within 5 days.

\section{Results:}

A total 11 PAF pts (age $62.8 \pm 13.0$ yrs; M / F = 5 / 6; LVEF $55 \pm 8.1 \%$; LA $41.5 \pm 5.8 \mathrm{~mm}$ ) underwent PVI. Using typically just one application per vein, PVI was acutely successful in 43 of $43(100 \%)$ PVs in 11 of $11 \mathrm{pts}(100 \%)$. The total pulse delivery period for each patient was $24 \pm 5$ seconds (range: 15 - 36 seconds). The PVI duration time (transpired from $1^{\text {st }}$ to last lesion) was $30.6 \pm 6.3 \mathrm{~min}$ (range 22.6- 42.5). The total LA (PFA) catheter dwell time was $51 \pm 7$ min (range 42 - 66). There were no safety events - including no esophageal fistula, stroke/TIA, phrenic injury or tamponade. EGD in was normal in 4 of 4 pts. Brain MRI was normal in 6 of 7 pts; one pt had DWI+/FLAIR- lesions.

\section{Conclusions:}

In this first-in-human study, the "single-shot" map-and-ablate spherical array PFA catheter was able to safely and effectively isolate PVs to treat paroxysmal AF.

AFS2022-06

\section{Uploaded File(s)}

Multielectrode Spherical Array PFA Catheter positioned at the LSPV ostium (fluoro), and the pre-PFA (top) and post-PFA (bottom) electrogram and voltage amplitude maps.
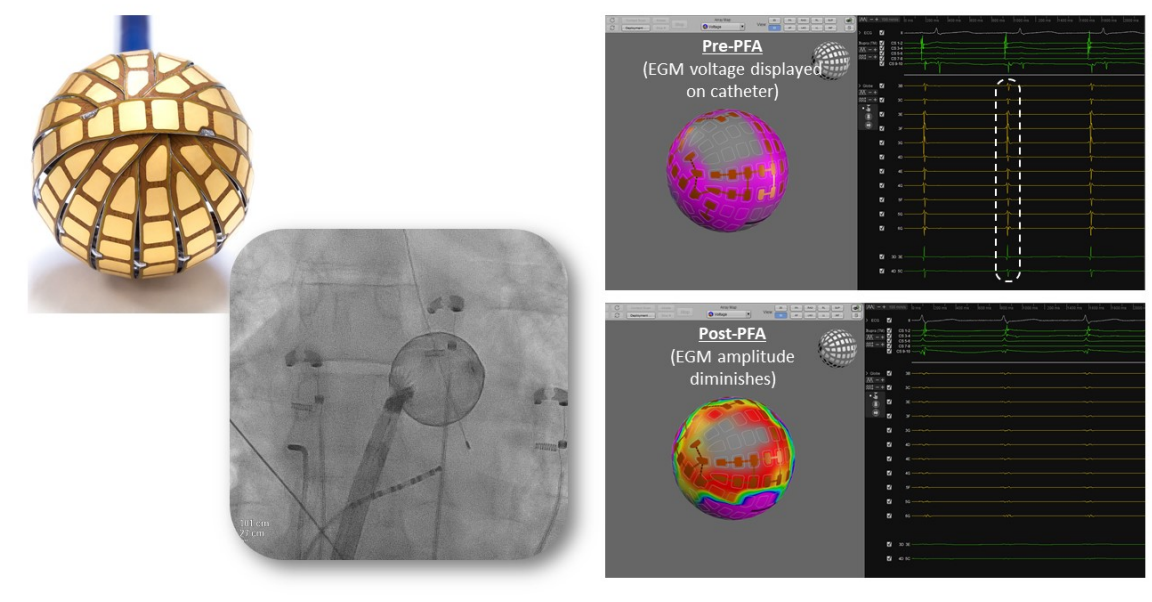

\section{AFS2022-07}

Abstract Title: First Clinical Experience with Epicardial Pulsed Field Ablation of Ganglionated Plexi During Cardiac Surgery

Author(s): Vivek Reddy, MD

Co-Author(s):

Ivo Skalsky, MD PhD

Tamaz Shaburishvilli, MD

Martin van Zyl, MD

Barry O'Brien, $\mathrm{PhD}$ 
Ken Coffey, BE

John Reilly, BE

Petr Neuzil, MD PhD

Joris de Groot, MD PhD

MOUNT SINAI MEDICAL CENTER

vivek reddy one gustave levy place, box 1030 mount sinai hospital

Introduction | Objectives:

The role of the autonomic nervous system in cardiac arrhythmias is increasingly being appreciated. However, studies of autonomic interventions have given mixed outcomes, particularly in the context of ganglionated plexi (GP) ablation for the treatment of atrial fibrillation. Selective GP ablation offers the potential for clearly elucidating the role of the GPs, by achieving GP neuronal cell destruction with minimal myocardial damage. This selective destruction of neuronal cells can be achieved through use of pulsed field ablation (PFA), with the energy applied directly to the epicardial fat pads in which the GPs are embedded. Herein, we present preliminary data from an ongoing first-in-human study (NCT 04775264) to evaluate the safety and feasibility of epicardial PFA to assess how atrial tissue refractoriness is influenced by this GP ablation.

\section{Methods:}

Atrial GPs were ablated in nineteen patients undergoing elective open-chest CABG surgery, using a monopolar, monophasic PFA system (AtriAN Medical). The Oblique Sinus GP, Right Superior GP, Transverse Sinus GP, Left Superior GP and Ligament of Marshall GP were each ablated with up to 60 ECG-gated PEF pulses of $1000 \mathrm{~V}$ amplitude and $100 \mu$ s pulse width. Atrial Effective Refractory Period (AERP) was measured epicardially at the left atrial appendage (LAA) and right atrial (RA) locations, before and after all ablations were completed. T-testing was used to compare pairs of pre- and post-PFA AERPs.

\section{Results:}

Successful GP ablations were completed in nineteen patients (mean age 61 years, $63 \%$ male) undergoing open-chest elective CABG surgery. An average AERP extension of $21 \%$ was obtained from thirteen valid pre- and post-ablation datasets (changed from $233 \pm 51 \mathrm{~ms}$ to $260 \pm 55 \mathrm{~ms} ; \mathrm{p}<0.01$ ). Patients progressed immediately to their planned elective surgery and were discharged on schedule. Three patients experienced post-operative AF.

\section{Conclusions:}

Selective pulsed field ablation of GPs in an open-chest setting has been demonstrated to be feasible and safe. An acute extension of atrial refractoriness is promising in terms of the potential to reduce AF recurrence. Further studies are required in symptomatic AF patients, using percutaneous epicardial access, to demonstrate long-term benefits.

AFS2022-07

Uploaded File(s)

GP Sites Targeted for PFA During Cardiac Surgery 


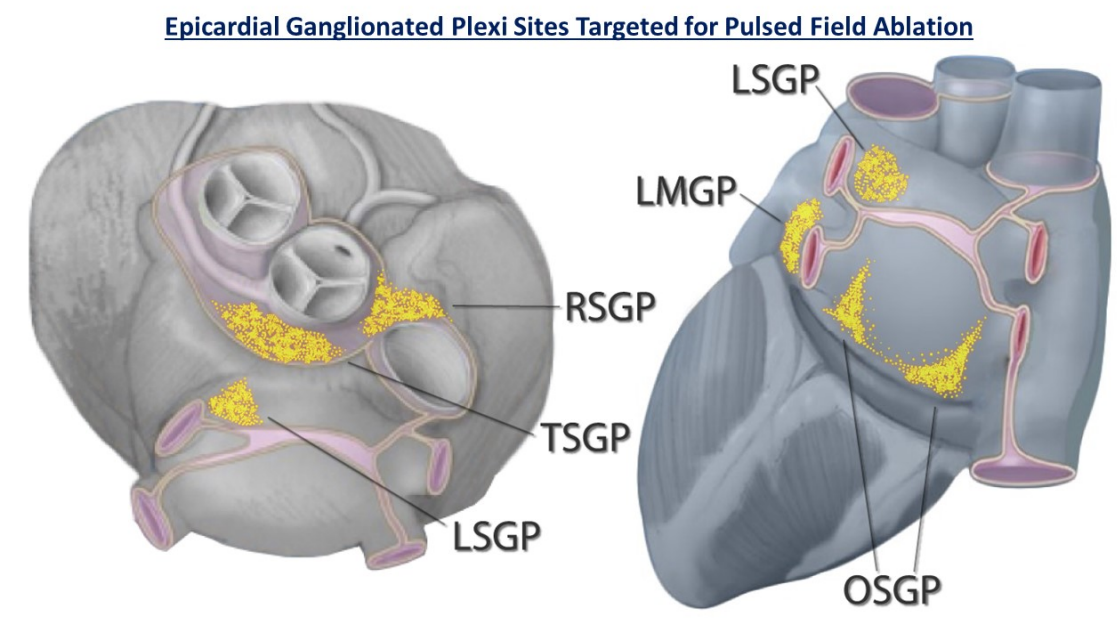

General Abstracts: AFS 2022-08 to -59

Best Abstract Award: AFS 2022 -08

AFS2022-08Submission Number: 1166750

Abstract Title: Lower pulmonary vein-to-left atrium volume ratio predicts poor rhythm outcome after atrial fibrillation catheter ablation

Author(s): Jae-Hyuk Lee, MD

Co-Author(s):

Inseok Hwang, BSc

Hee Tae Yu, MD, PhD

Tae-Hoon Kim, MD

Jae-Sun Uhm, MD, PhD

Boyoung Joung, MD, PhD

Moon-Hyoung Lee, MD, PhD

Hui-Nam Pak, MD, PhD - Yonsei University Health System

Severance hospital

50-1 Yonsei-ro, Seodaemun-gu, Seoul 03722, Republic of Korea

Introduction | Objectives: Although left atrial (LA) dimension (LAD) is one of the predictors of atrial fibrillation $(\mathrm{AF})$ recurrence after catheter ablation, repetitive recurrences occur in patients withot enlarged LAD. We explored the predictive value of the pulmonary vein (PV) to LA volume percent ratio (PV/LA\%vol) for rhythm outcomes of AF catheter ablation (AFCA).

Methods: We included 2913 patients (73.5\% male, 60.0 [52.0-67.0] years old, 60.6\% with paroxysmal $\mathrm{AF}$ ) who underwent AFCA. We additionally investigated the association between the PITX2 gene and PV/LA\%vol in 2051 patients using a genome-wide association study.

\section{Results:}


LAD affected 1-year recurrence only in the highest tertile group (T3, $\mathrm{p}=0.046$ ), but PV/LA\%vol determined 1-year recurrence in all T1-T3 LAD groups (T1, $\mathrm{p}=0.044 ; \mathrm{T} 2, \mathrm{p}=0.021$; and $\mathrm{T} 3, \mathrm{p}=0.045)$. During 20.0 (8.0-45.0) months of follow-up, the clinical recurrence rate was significantly higher in patients with lower PV/LA\%vol (T1-T3, Log-rank $\mathrm{p}=0.004$, hazard ratio $[\mathrm{HR}] 0.91[0.84-1.00], \mathrm{p}=0.044$ ). In the T1 and T2 LAD groups, predicting AF recurrences was significantly better with PV/LA\%vol than with LAD (area under the receiver operating characteristic curve [AUC] 0.63 vs. $0.51, \mathrm{p}<0.001$ at T1; AUC 0.61 vs. $0.50, \mathrm{p}=0.007$ at T2). We replicated a PITX2-related rs12646447, which was independently associated with PV/LA\%vol $(\beta=0.15$ [0-0.30], $\mathrm{p}=0.047)$ but not the risk of AF recurrence after AFCA (HR 0.93 [0.80-1.09], $\mathrm{p}=0.371)$.

Conclusions: Smaller PV volumes after LA volume adjustments have genetic background of PITX2 gene and predictive value for poorer rhythm outcomes after AFCA, especially in patients without significant LA enlargement.

\section{AFS2022-08 Uploaded File(s)}

The novel parameter, PV/LA\%vol, for predicting rhythm outcomes after AFCA, especially in patients with a normal or small LA dimension.
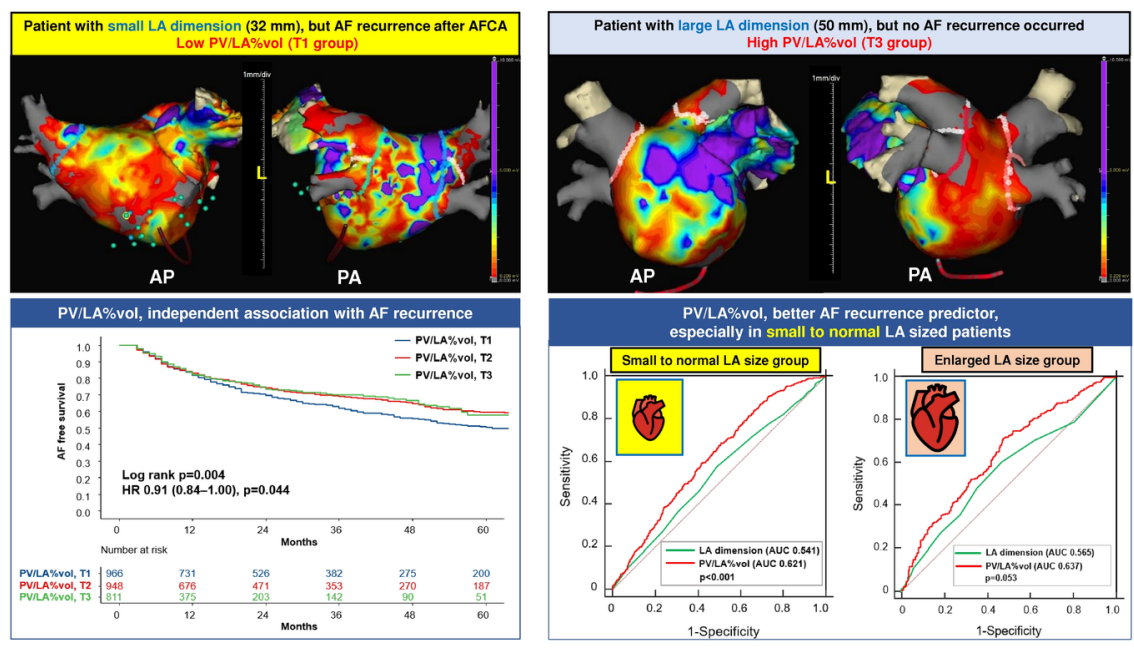

\section{AFS2022-09 Submission Number: 1174278}

Abstract Title: Use of the Novel AcQCross Transseptal System Improves Procedural Efficiency Compared to Conventional Transseptal Puncture Technique

Author(s): Sang Yong Ji, M.D.

Co-Author(s):

Gene Kim, M.D. - Cardiac Electrophysiologist, Torrance Memorial Medical Center

Erol Kosar, M.D. - Cardiac Electrophysiologist, Torrance Memorial Medical Center

Aaron DeTate, M.D. - Cardiac Electrophysiologist, Torrance Memorial Medical Center

Morse Stephen, Cardiovascular Technician - Cardiovascular Technician, Torrance Memorial Medical Center

Terrones Laura, MPH - Clinical Data Specialist, Acutus Medical Inc

Matthew Ostrom, M.D. - Cardiac Electrophysiologist, Torrance Memorial Medical Center

Torrance Memorial Medical Center 
3330 Lomita Blvd, Central Tower, 1st floor, Division of Cardiac Electrophysiology Torrance, CA 90505

Introduction | Objectives:

Background: Transseptal (TS) puncture can be one of the most stressful and unpredictable steps in the procedural workflow in a variety of electrophysiology and structural left heart procedures. The novel AcQCross System (Acutus Medical) offers a fixed or steerable sheath paired with an integrated needleand-dilator device that retains the guidewire during positioning, re-positioning and puncture to reduce the number of exchanges required during TS puncture (Panel A).

Objective: Compare the safety and efficiency of the novel AcQCross TS system (AQC) to the standard of care (SOC) TS crossing platform (Abbott BRK + SL0).

\section{Methods:}

Methods: Retrospective analysis of TS punctures performed during AF ablation procedures between 20152020 in a single, high volume ablation center with multiple operators $(n=4)$. One hundred $(100)$ consecutive AcQCross TS patients were compared with 100 consecutive SOC TS patients. Measurable outcomes included

Efficiency $=$ Time of TS sheath/needle entry into the venous access site to Time of TS sheath/needle entry into LA Safety = proportion of patients free of acute device/procedure related Serious Adverse Events (SAE).

\section{Results:}

Results: Patient age in the AcQCross arm was $67.1 \pm 10.1$ v $67.0 \pm 11.4$ years for SOC $(\mathrm{p}=.93), 64 \%$ v $80 \%$ were male $(\mathrm{p}=.01)$, respectively. The mean time for AcQCross was 9 min, $10 \sec ( \pm 9: 31)$ vs 16 min, $16 \sec ( \pm 23: 51)$ for SOC, yielding a $43.6 \%(\mathrm{p}=.006)$ greater efficiency. No major safety issues were reported. The Procedural variables are presented in panel B and outcomes in Panel C.

\section{Conclusions:}

Conclusions: In a large single center retrospective study and independent of physician operator, the AcQCross System was more efficient and more predictable in time to TS crossing compared to SOC. The safety profile was similar.

\section{AFS2022-09}

\section{Uploaded File(s)}

\section{A.Figure B.Table C.Graph}

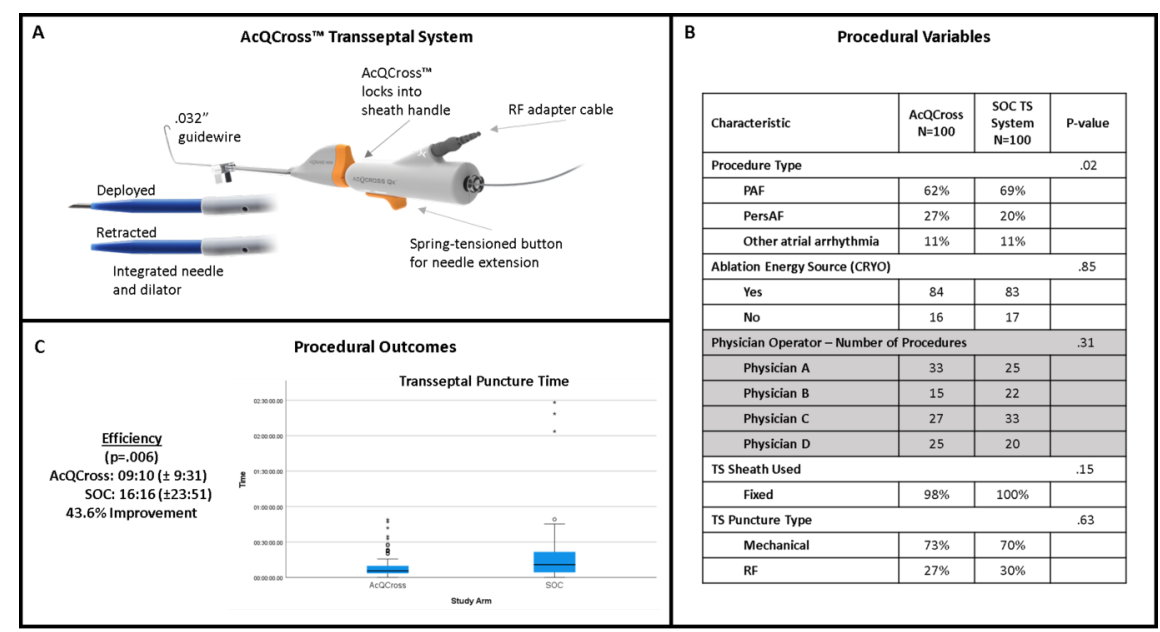

AFS2022-10Submission Number: 1164109 
Abstract Title: A Multicenter, Multinational Exploration of the Contribution of Discrete Intraprocedural Segments to Overall Fluoroscopy Use During Atrial Fibrillation Ablation

Author(s): Saumil R. Shah, MD, FHRS

Co-Author(s):

Yun-Gi Kim, MD - Korea University Medical Center

Jose Martel, MD, MPH, FHRS - South Miami Hospital

Kamel Addo, MD - Mount Carmel St. Ann's

David DeLurgio, MD - Emory St. Joseph's

Hany Demo, MD - Swedish Hospital

Firas Hamdan, MD - Aultman Hospital

Gregory Bonavita, MD - Aultman Hospital

F. Kevin Hackett, MD - Mount Carmel Health

Christopher Bailey, MBA - Abbott

Jae-min Shim, MD - Korea University Medical Center

Virginia Arrhythmia Consultants

1001 Boulders Parkway, Suite 110, Richmond, VA, 23225

Introduction | Objectives: Reduction of radiation exposure in atrial fibrillation (AF) ablation procedures has become an area of increasing focus in recent years. While total fluoroscopy time is a commonly reported metric, the specific intraprocedural steps that drive overall use are not well studied, and thus may provide insights into where there are opportunities to further reduce the harmful effects of ionizing radiation. The objective is to examine trends in fluoroscopy use during atrial fibrillation (AF) ablation, including individual procedural steps that drive overall fluoroscopy use during ablation of AF.

Methods: Acute procedure data from 649 AF cases was prospectively collected from 75 centers in nine countries. Procedure workflow, use of technology, and fluoroscopy use were left to physician discretion. Fluoroscopy times for the entire procedure, including intraprocedural steps driving overall use, were recorded in all cases.

Results: Of the 649 cases, de novo paroxysmal AF (PAF) was the most common indication (336 cases; $52 \%$ ) (Table 1). Across all cases, an average total fluoroscopy time of $14.9 \pm 17.6$ minutes was observed, with use during ablation accounting for $41.6 \%$ of the total fluoroscopy time (6.2 $\pm 10.0 \mathrm{~min})$. Following ablation, segments accounting for fluoroscopy usage were procedure close $(2.9 \pm 10.2 \mathrm{~min})$, mapping $(2.3 \pm 3.7 \mathrm{~min})$, transseptal puncture $(1.9 \pm 3.6 \mathrm{~min})$, and initial catheter placement $(1.8 \pm 3.5 \mathrm{~min})$. Usage patterns across various sub-indications are shown in Table 1.

Conclusions: Usage patterns were relatively consistent across AF sub-indications; LsPersAF appeared to be an outlier although that may have been driven by small sample size. Ablation had the greatest impact on overall fluoroscopy use, accounting for nearly half of the total fluoroscopy time. Future efforts to minimize radiation exposure may be well-served by focusing on reducing use during ablation, potentially by embracing alternative imaging modalities such as 3D mapping technologies and intracardiac echocardiography (ICE). Further analysis is needed to compare and quantify the impact that technology use and workflow preferences may have on reducing fluoroscopy use in AF cases, notably during ablation.

\section{AFS2022-10}

Uploaded File(s) 
Table 1. Intraprocedural and total fluoroscopy times during cardiac ablation of atrial fibrillation.

\begin{tabular}{|c|c|c|c|c|c|c|c|}
\hline Indication & $n$ (\%) & $\begin{array}{c}\text { Average } \\
\text { Fluoroscopy Time: } \\
\text { Catheter Placement } \\
\text { (min) }\end{array}$ & $\begin{array}{c}\text { Average } \\
\text { Fluoroscopy Time: } \\
\text { Transseptal (min) }\end{array}$ & $\begin{array}{c}\text { Average } \\
\text { Fluoroscopy Time: } \\
\text { Mapping } \\
\text { (min) }\end{array}$ & $\begin{array}{c}\text { Average } \\
\text { Fluoroscopy Time: } \\
\text { Ablation } \\
\text { (min) }\end{array}$ & $\begin{array}{c}\text { Average } \\
\text { Fluoroscopy Time: } \\
\text { Procedure Close } \\
\text { (min) }\end{array}$ & $\begin{array}{l}\text { Average Total } \\
\text { Fluoroscopy Time } \\
\text { (min) }\end{array}$ \\
\hline $\begin{array}{l}\text { De Novo } \\
\text { Paroxysmal AF }\end{array}$ & 336 (52) & $1.7 \pm 3.7$ & $2.0 \pm 3.8$ & $2.3 \pm 3.9$ & $5.0 \pm 9.4$ & $4.0 \pm 13.4$ & $14.7 \pm 20.3$ \\
\hline $\begin{array}{l}\text { De Novo } \\
\text { Persistent AF }\end{array}$ & 171 (26) & $2.2 \pm 3.6$ & $2.0 \pm 3.7$ & $2.4 \pm 4.0$ & $8.8 \pm 11.2$ & $1.4 \pm 4.2$ & $16.6 \pm 14.8$ \\
\hline $\begin{array}{l}\text { Longstanding } \\
\text { Persistent AF }\end{array}$ & $3(1)$ & $1.3 \pm 1.8$ & $3.9 \pm 5.0$ & $3.2 \pm 3.1$ & $2.5 \pm 2.2$ & $8.9 \pm 13.9$ & $19.7 \pm 19.1$ \\
\hline $\begin{array}{l}\text { Redo } \\
\text { Paroxysmal AF }\end{array}$ & $65(10)$ & $1.5 \pm 3.4$ & $1.3 \pm 2.6$ & $1.6 \pm 2.7$ & $5.1 \pm 8.8$ & $2.6 \pm 6.4$ & $11.9 \pm 12.9$ \\
\hline $\begin{array}{l}\text { Redo } \\
\text { Persistent AF }\end{array}$ & 74 (11) & $1.9 \pm 2.5$ & $1.8 \pm 2.0$ & $2.7 \pm 3.0$ & $7.3 \pm 9.4$ & $0.7 \pm 3.1$ & $14.4 \pm 13.1$ \\
\hline Grand Total & 649 & $1.8 \pm 3.5$ & $1.9 \pm 3.6$ & $2.3 \pm 3.7$ & $6.2 \pm 10.0$ & $2.9 \pm 10.2$ & $14.9 \pm 17.6$ \\
\hline
\end{tabular}

\section{AFS2022-11Submission Number: 1164538}

Abstract Title: Safety and Feasibility of Radiofrequency Ablation Following Implant of the Amulet Left Atrial Appendage Occluder (Perspective from the Amulet IDE Trial)

Author(s): Christopher R. Ellis, MD, FACC, FHRS

Co-Author(s):

Vanderbilt Heart Institute

1215 21st Avenue South, 5th Floor MCE 5414 Nashville, TN 37232

\section{Introduction | Objectives:}

\section{Introduction}

Ablation performed in paroxysmal atrial fibrillation $(\mathrm{AF})$ patients post left atrial appendage occlusion (LAAO) may present unique challenges maneuvering around an LAAO device. Risks of ablation post-LAAO include device dislodgement, device perforation, thrombus formation, and incomplete ablation. There are few reported cases of ablation performed after the implantation of an Amplatzer ${ }^{\mathrm{TM}}$ Amulet ${ }^{\mathrm{TM}}$ LAA Occluder.

\section{Objective}

Demonstrate the safety and feasibility of radiofrequency (RF) ablation after implant of an Amulet LAA occluder.

\section{Methods:}

Six paroxysmal AF patients enrolled in the Amulet IDE Trial at Vanderbilt University Medical Center underwent planned RF ablation post-implant of Amulet occluder using CARTOSOUND and ICE. In all cases, the ablation was performed past the 45-day required TEE follow-up period (607 \pm 321 days). Lesion sets included wide area PVI plus additional linear ablation when indicated.

\section{Results:}

Patients ranged in age (69.2 \pm 7.4 years), sex ( $50 \%$ female), $\mathrm{CHA}_{2} \mathrm{DS}_{2}$-VASc score $(4.8 \pm 1.5)$, HAS-BLED score $(3.0 \pm 0.9)$, and medical history prior to LAAO ( 2 with prior stroke; 3 with prior major bleeding). In all cases, technical implant success was observed with a wide range of Amulet occluder sizes implanted (lobe: $18-31 \mathrm{~mm}$; disc: $24-38 \mathrm{~mm}$ ). Left atrial ICE was used to directly observe disc position and catheter location during RF. After ablation, patients were discharged on single antiplatelet therapy $(\mathrm{n}=3)$, dual antiplatelet therapy $(n=1)$, and oral anticoagulation $(n=2)$. In all cases, no ablation-related adverse events occurred with no repeat procedures through 1-year post-ablation. There were no reported peridevice leaks $([?] 3 \mathrm{~mm})$, device-related thrombus, device embolizations, or major clinical adverse events (stroke, major 
bleeding, pericardial effusion, or death) in any patients 1-year post-ablation. One patient experienced sinus bradycardia arrhythmia 50 days after ablation which resulted in a pacemaker implant.

\section{Conclusions:}

In conclusion, RF ablation post-implantation of the Amulet ${ }^{\mathrm{TM}}$ occluder can safely and successfully be performed to facilitate restoration of sinus rhythm with imaging-based guidance to avoid damage to the LAAO device.

\section{AFS2022-12Submission Number: 1164558}

Abstract Title: Proactive esophageal cooling during radiofrequency ablation is associated with improved one-year freedom from atrial fibrillation

Author(s): Jason Zagrodzky, MD

Co-Author(s):

William Zagrodzky, BS - Colorado College

Christopher Joseph, BA

Shane Bailey, MD

Erik Kulstad, MD, MS - UT Southwestern Medical Center

Texas Cardiac Arrhythmia Institute

901 W Ben White Blvd, Austin, TX 78704

Introduction | Objectives: Proactive esophageal cooling during pulmonary vein isolation (PVI) is increasingly being used with radiofrequency (RF) ablation for the treatment of atrial fibrillation (AF). Because proactive cooling avoids heat stacking, thereby allowing continuous point-to-point ablation, transmurality and continuity of lesions may be enhanced, thereby improving long-term efficacy in freedom from atrial arrhythmias. We evaluated this effect by analyzing one-year rhythm status in patients treated with proactive esophageal cooling and comparing the results to patients treated with luminal esophageal temperature (LET) monitoring.

Methods: We reviewed data of patients treated before and after the implementation of esophageal cooling at a single center. Patient rhythm status at one-year follow up after receiving PVI for the treatment of AF was recorded. Patients were dichotomized as being in normal sinus rhythm versus any atrial arrhythmia (AF, atrial flutter, atrial tachycardia). Results were compared between patients receiving active esophageal cooling and those treated with traditional LET monitoring using Kaplan-Meier estimates.

Results: A total of 168 patients had follow-up data for review. Of these, 68 received LET monitoring using primarily single-sensor temperature probes, and 100 received active cooling. Mean age was 67 (range 30 to 87) years, and $32.1 \%$ were female. At one-year follow-up, KM estimates for freedom from AF were $71.8 \%$ for LET monitored patients and $93.0 \%$ for actively cooled patients, representing an absolute increase in freedom from $\mathrm{AF}$ of $14 \%$ with active esophageal cooling $(\mathrm{P}=.045)$.

Conclusions: Patients treated with proactive esophageal cooling during PVI had greater freedom from atrial arrhythmias at one year when compared to patients treated with LET monitoring. This effect may be a result of improving the ability to place point-to-point lesions without interruption or pauses when proactive cooling is employed.

\section{AFS2022-12}

Uploaded File(s)

Proactive esophageal cooling during radiofrequency ablation is associated with improved oneyear freedom from atrial fibrillation 


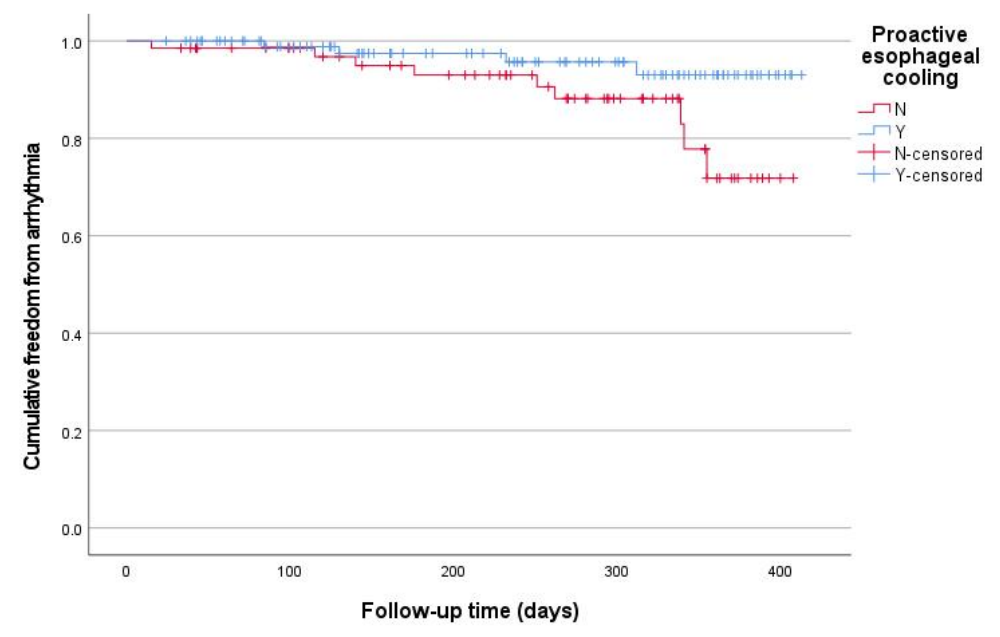

AFS2022-13Submission Number: 1165330

Abstract Title: Left Atrial Functions In The Early Period After Cryoballoon Ablation For Paroxysmal Atrial Fibrillation

Author(s): Ali Ugur Soysal, n/a

Co-Author(s):

Zafer Akman, MD

Ali Egemen Koroglu, MD

Tolga Aksu, MD

Adem Atici, MD

Ebru Golcuk, MD

Hasan Tokdil, MD

Hakan Yalman, MD

Baris Ikitimur, MD

Kivanc Yalin, MD

Istanbul University-Cerrahpasa, Cerrahpasa Faculty of Medicine

Cerrahpasa, Koca Mustafapasa street. Fatih/Istanbul

Introduction | Objectives: In patients with paroxysmal atrial fibrillation (PAF), functional changes are observed in the left atrium (LA) after pulmonary vein isolation (PVI) with catheter ablation. Although previous studies have investigated the altered mechanical functions of LA with radiofrequency (RF) ablation, changes in LA functions in the early period after cryoablation have not been clearly demonstrated.

\section{Methods:}

Thirty three patients (mean age: $57 \pm 11 ; 54.5 \%$ men) with PAF underwent cryoablation procedure with second generation cryoballoon (CB-2). All patients were in sinus rhythm before and after the procedure. LA dimensions, LA strain parameters (LAsr-LAscd-LAsct) and left ventricular diastolic function parameters were evaluated by Doppler echocardiography before and 3 months after the procedure. 
Results: Acute procedural success was achieved in all cases. No major complications were occurred. A significant recovery in LA reservoir strain $(p=0.009)$ was observed. No significant change was demonstrated in remaining echocardiographic parameters.

Conclusions: LA reservoir strain is an indicator of left atrial compliance and significant improvement in mechanical functions may occur even in the early period after cryoballoon ablation in patients with PAF in contrast to LA function deterioration observed in RF ablation.

\section{AFS2022-13}

\section{Uploaded File(s)}

\begin{tabular}{|c|c|c|c|}
\hline & $\begin{array}{l}\text { Pre-procedure (mean) } \\
(n=33)\end{array}$ & $\begin{array}{c}\text { 3rd-month (mean) } \\
(n=33)\end{array}$ & P-value \\
\hline LA diameter $(\mathrm{mm} \pm \mathrm{SD})$ & $40,24 \pm 6,42$ & $40,39 \pm 6,47$ & 0,096 \\
\hline $\operatorname{LAVI}(\mathrm{mL} / \mathrm{m} 2 \pm \mathrm{SD})$ & $29,16 \pm 13,89$ & $30,0113,34$ & 0,398 \\
\hline LA strain - LASR $(\% \pm S D)$ & $27,79 \pm 13,31$ & $33,08 \pm 14,03$ & 0,009 \\
\hline LA strain - LASCD (\% $(S D)$ & $-18,55 \pm 9,64$ & $-17,12 \pm 11,44$ & 0,520 \\
\hline LA strain - LASCT (\% $\pm S D)$ & $-10,31 \pm 8,62$ & $-13,08 \pm 11,6$ & 0,245 \\
\hline LV endocardial average strain $(\% \pm S D)$ & $-21,8 \pm 14,39$ & $-19,49 \pm 11,45$ & 0,486 \\
\hline LA-EMT lateral $(m s n \pm S D)$ & $94,73 \pm 22,37$ & $94,67 \pm 21,64$ & 0,977 \\
\hline LA-EMT medial $(m s n \pm S D)$ & $77,8 \pm 22,46$ & $79,07 \pm 23,83$ & 0,738 \\
\hline E-wave velocity $(\mathrm{m} / \mathrm{sn} \pm \mathrm{SD})$ & $80,93 \pm 22,76$ & $84,68 \pm 21,66$ & 0,240 \\
\hline A-wave velocity $(\mathrm{m} / \mathrm{sn} \pm \mathrm{SD})$ & $69,56 \pm 18,12$ & $67,83 \pm 21$ & 0,684 \\
\hline $\operatorname{TYV}(\mathrm{m} / \mathrm{sn} \pm \mathrm{SD})$ & $2,39 \pm 0,27$ & $2,44 \pm 0,33$ & 0,382 \\
\hline Medial E/e' & $10,96 \pm 3,93$ & $11,47 \pm 4,52$ & 0,335 \\
\hline Lateral $\mathrm{E} / \mathrm{e}^{\prime}$ & $8,03 \pm 3,15$ & $8,52 \pm 2,94$ & 0,151 \\
\hline Average $\mathrm{E} / \mathrm{e}^{\prime}$ & $9,62 \pm 3,27$ & $10,45 \pm 3,79$ & 0,140 \\
\hline
\end{tabular}

Significant P-values are indicated in bold characters

\section{AFS2022-14Submission Number: 1165606}

Abstract Title: Comparison of the results of pulmonary vein isolation in patients younger than and older than 70 years

Author(s): Gustavo Maid, MD

Co-Author(s):

Santiago Bustamante, MD

pablo costa, MD

florencia duckwen, MD

gonzalo fernandez Villar, MD

rodolfo pizarro, MD

emiliano rossi, MD

juan vergara, MD

Hospital Italiano de Buenos Aires

Gascon 450

Introduction | Objectives: to compare the clinical characteristics and the results of contemporary cohorts of patients younger and older than 70 years who underwent ablation of AF by catheter. 
Methods: Retrospective cohort study of patients undergoing the first catheter ablation procedure for AF refractory to antiarrhythmic drugs with a minimum follow-up per patient of 12 months.

Results: Results: 239 patients undergoing AF ablation were included in the study: $171(71.5 \%)$ in the < 70 years group and $68(28.5 \%)$ in the $>70$ years group. The mean age of the study population was 62.4 (SD 10.87), the group $<70$ years had an average age of 58.03 years (SD 9.71), the group $>70$ years had a average age of 73.4 years (SD 3.05). There were no differences between the groups with respect to body mass index, the anteroposterior diameter of the left atrium and comorbidities such as diabetes mellitus, heart failure, coronary artery disease, and a previous history of cerebrovascular accident / TIA, except in HT and coronary artery disease, where the percentage was higher among patients older than 70 years. There were also no significant differences between the groups in the proportion of use of ACE inhibitors / ARB-2 and antiarrhythmic drugs, except for flecainide which was used in a higher proportion in patients younger than 70 years. A higher proportion of paroxysmal AF was observed in patients $<70$ years and a higher proportion of persistent AF in patients $>70$ years. The difference being statistically significant in both cases. Recurrence rates after the first ablation procedure were similar between the groups $(21.43 \%$ group $<70$ years vs $23.53 \%$ group $>70$ years, $\mathrm{p}=0.79)$. There were also no significant differences in terms of complications. Complications of the group $<70$ years were 18, complications of the group $>70$ years were $5, \mathrm{p}=0.472$. There was no difference in the time of hospitalization of the patients (days) categorized by age. Throughout the study, no atrioesophageal fistulas, TIA / stroke, or death after ablation were reported.

Conclusions: Patients older than 70 years who undergo the first AF catheter ablation procedure would present similar clinical results to patients younger than 70 years.

AFS2022-14

Uploaded File(s)

AF recurrence-free survival during the first 12 months

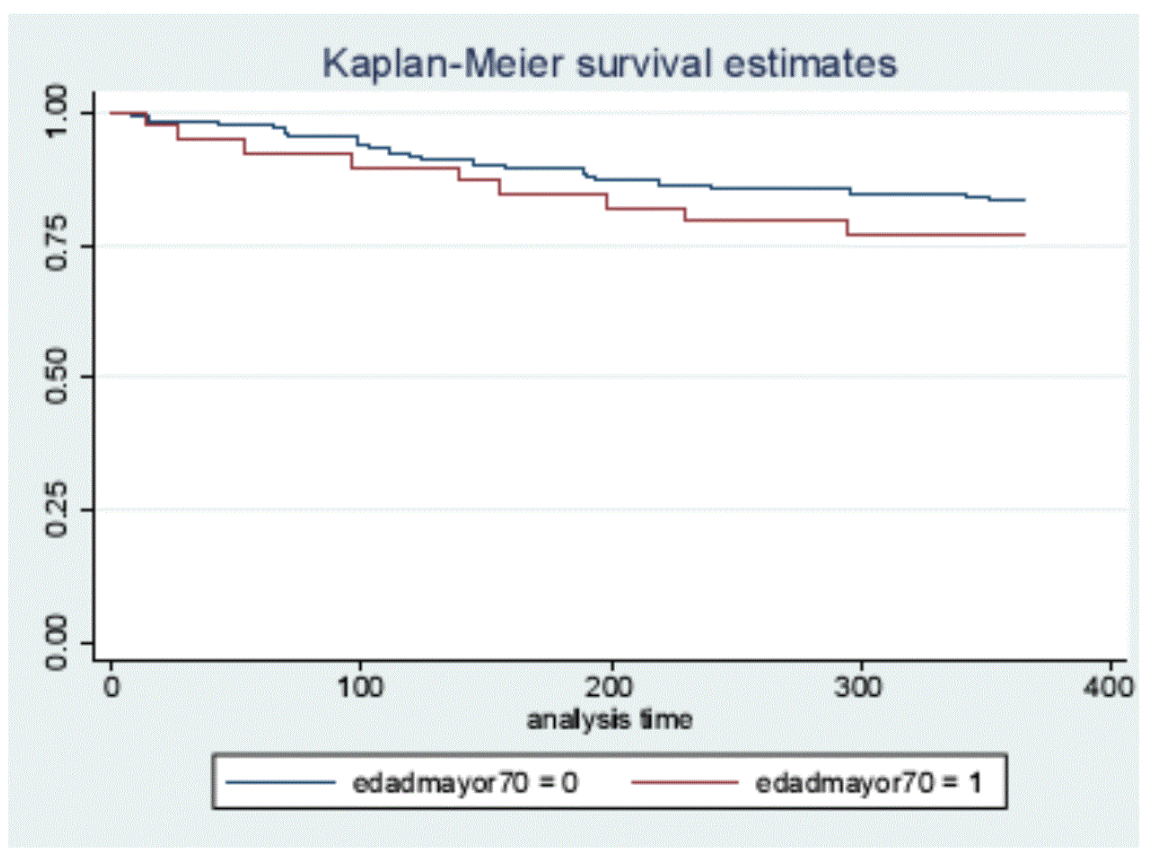

AFS2022-15Submission Number: 1165942

Abstract Title: Temperature profiles with and without proactive esophageal cooling during high-power 
short-duration radiofrequency ablation

Author(s): Marcela Mercado-Montoya, MSC

Co-Author(s):

Tatiana Gomez-Bustamante, BS

Enrique Berjano, $\mathrm{PhD}$

James Daniels, MD

Steven Mickelsen, MD

Pablo Hernandez-Arango, MSc

Jay Schieber, $\mathrm{PhD}$

Erik Kulstad, MD, MS - UT Southwestern Medical Center

In Silico Science \& Engineering S.A.S

Cl. 52 \#81-48, Medellín, Antioquia, Colombia

Introduction | Objectives: Proactive esophageal cooling reduces severe esophageal thermal injury during medium-power medium-duration radiofrequency (RF) ablation. Proactive esophageal cooling has increasingly been used during high-power short-duration ablation (HPSD), with thousands of cases completed without an atrioesophageal fistula (AEF) reported, but models of cooling under HPSD ablation have not yet been published. We sought to determine temperature profiles across ablated tissues during HPSD ablation with and without proactive cooling to measure the impact of cooling on esophageal temperature.

Methods: We created a computer model of the left atrium and esophagus and used this to determine temperature profiles from the RF ablation catheter to the esophageal lumen under two HPSD ablation conditions $(50 \mathrm{~W}$ for $10 \mathrm{~s}$ and $90 \mathrm{~W}$ for $4 \mathrm{~s})$. We then determined temperature profiles with proactive cooling in place, and measured the effect on esophageal temperatures from endoluminal to epi-esophageal surface.

Results: Temperatures exceeded the lethal isotherm temperatures $\left(50^{\circ} \mathrm{C}\right)$ across the majority of the wall of the esophagus under both $50 \mathrm{~W}$ and $90 \mathrm{~W}$ ablation conditions without proactive cooling. With proactive esophageal cooling present, temperatures in the esophageal tissue were reduced across the esophageal wall, with the maximum mucosal surface temperature reduced to $22^{\circ} \mathrm{C}$, representing a reduction of $44 \%$, and remaining far below temperatures needed to induce tissue damage.

Conclusions: Proactive esophageal cooling significantly reduced temperatures in the esophagus during HPSD ablation using both $50 \mathrm{~W}$ and $90 \mathrm{~W}$ power settings. This provides further understanding of the clinical effects reported to date on this method of esophageal protection during HPSD ablation.

AFS2022-15

Uploaded File(s)

Temperature profiles with and without proactive esophageal cooling during high-power shortduration radiofrequency ablation 


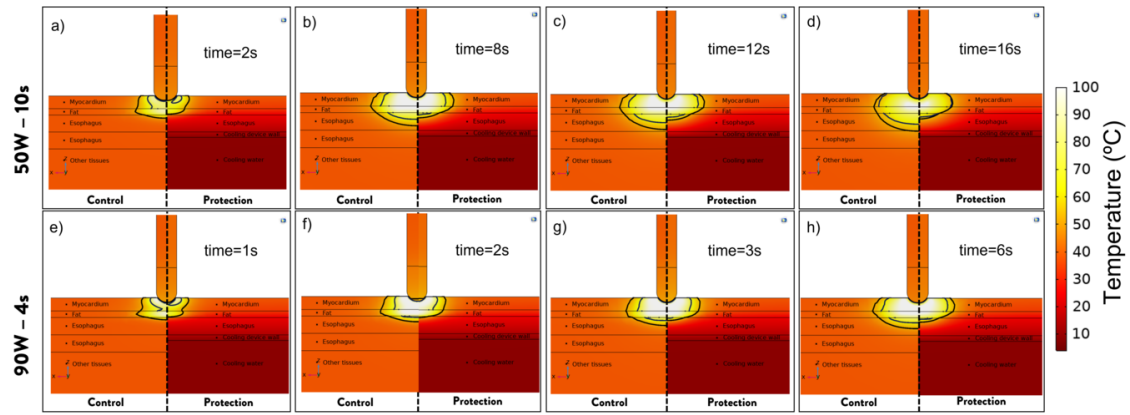

\section{AFS2022-16Submission Number: 1165945}

Abstract Title: Mechanisms of action behind proactive esophageal cooling during left atrial ablation

Author(s): Shadi Kalantarian, MD

Co-Author(s):

Audra Clark, MD

Kamala Tamirisa, MD

Jason Zagrodzky, MD

James Daniels, MD

Chris Joseph, BA

Erik Kulstad, MD, MS - UT Southwestern Medical Center

Ben Levi, MD

\section{$\mathrm{UCSF}$}

400 Parnassus Ave Fifth Floor, San Francisco, CA 94143

Introduction | Objectives: Proactive esophageal cooling during left atrial ablation reduces severe esophageal injury and may even prevent formation of potentially lethal atrioesophageal fistulas (AEFs). Direct heat dissipation is one proposed mechanism of effect; however, with thousands of uses to date of proactive esophageal cooling without AEF development, other mechanisms may contribute to this outcome. To identify such mechanisms, we sought to review the growing data on the cooling of thermal burns and the effects of this therapy.

Methods: We conducted a literature review to identify studies describing the pathophysiology of thermal injury and the effects of cooling. We searched the PubMed/Medline database with the MeSH term "Burns/therapy" [MH] AND (cool* OR cold*) and retrieved additional publications using the MEDLINE option "Similar articles".

Results: A total of 427 articles were identified, of which 219 were excluded due to inapplicability. Of the remaining 208 reviewed, the benefits of cooling included decreased injury progression, depth, severity, grafting requirements, and time to healing, with dose-response effects depending on time delay before initiation and on duration of cooling. Shorter delays, and longer durations (to a threshold of 20 minutes) provided greater benefits. Mechanisms of cooling benefits included (i) decreasing release of lactate and histamine, (ii) stabilizing thromboxane and prostaglandin levels, (iii) slowing local metabolism, (iv) reducing membrane permeability, (v) inhibiting kallikrein activity, and (vi) changing gene expression in burned tissues. Delayed cooling of up to 3 hours (long after tissue temperatures have normalized) also resulted in improved outcomes. 
Conclusions: The data indicate that numerous mechanisms in addition to heat dissipation contribute to the benefits of cooling, and that an additional mechanism leading to the beneficial effects of proactive esophageal cooling is the direct inhibition of the inflammatory response caused by thermal injury. This later-onset effect attenuates progression of thermal injury, and may provide further protection against AEF development.

AFS2022-16

\section{Uploaded File(s)}

Mechanisms of action behind proactive esophageal cooling during left atrial ablation

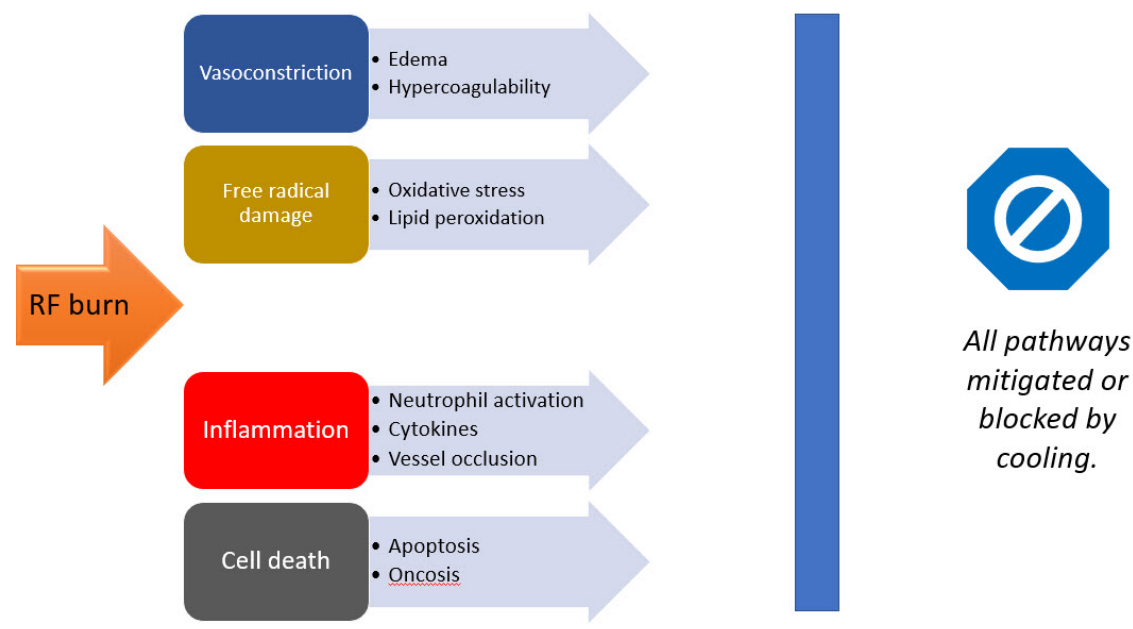

AFS2022-17Submission Number: 1166137

Abstract Title: Impact of WATCHMAN FLX and Amulet Devices on Left Atrial Appendage Adjacent Structures and Healing Response in a Canine Model

Author(s): Rodney Horton, MD

Co-Author(s):

Saibal Kar, MD - Los Robles Hospital

Srikara Viswanath Peelukhana, PhD - Boston Scientific

Thomas Herbst, PhD - Boston Scientific

Eric Dinges, MS - Boston Scientific

Jason Kilvington, BS - Boston Scientific

Matthew Palin, MS - Boston Scientific

Erin Hynes, MS - Boston Scientific

Brad Sutton, MD - Boston Scientific

Texas Cardiac Arrhythmia

3000 N IH35, Suite 700 Austin TX 78705 United States of America

Introduction | Objectives: 
A canine left atrial appendage (LAA) study $(\mathrm{n}=10)$ was designed to evaluate the healing response and impact to adjacent structures of the Watchman FLX (FLX) (Boston Scientific, Marlborough, Massachusetts) and Amulet (AM) (St. Jude Medical, Minneapolis, Minnesota). Implantation in the canine LAA was performed $(\mathrm{n}=5$ per device) under trans-esophageal echocardiography (TEE) and fluoroscopy guidance.

\section{Methods:}

The healing response, device conformation to the LA anatomy, transfabric flow and peri-device leak were evaluated by CT, TEE, gross examination after necropsy, and histology.

\section{Results:}

All devices were successfully implanted. At 45-days, on CT, FLX devices resulted in lower transfabric flow ( $1 / 5 \mathrm{FLX}$ vs $4 / 5 \mathrm{AMs}$ ), and peri-device leaks (0/5 FLX vs $2 / 5 \mathrm{AMs}, 4 \mathrm{~mm}$ and $4.4 \mathrm{~mm}$ at disk level). Upon gross examination at 45 -days, the exterior of the LAA was perforated by anchors in $2 / 5 \mathrm{AM}$ implants and 0/5 FLX implants. En-face estimates of neoendovardial (NeoE) tissue showed $0 / 5$ of the AM discs were $100 \%$ covered by a maturing neoendocardium (range 25\%-80\%) while 3/5 FLX devices were $100 \%$ covered by NeoE (range 25\%-100\%). The single FLX device with low tissue coverage (25\%) was implanted very proximal protruding into the atrial cavity with little native endocardial surface contact. Mitral valve (MV) impingement and/or PV compression were observed in all 5 of the AMs while one proximally placed FLX resulted in MV interaction. One AM resulted in device related mitral regurgitation.

\section{Conclusions:}

In conclusion, in this small comparative study in a canine model, the WATCHMAN FLX device was associated with fewer anchor perforations and less interaction with LAA adjacent structures than the Amulet device. This in combination with less transfabric flow and less peridevice leak might have impacted healing, as neo-endocardial coverage was near complete in the group treated with WATCHMAN FLX while all Amulet cases showed incomplete endothelialization.

AFS2022-18 Submission Number: 1167547

Abstract Title: A new score for predicting results after catheter ablation for atrial fibrillation: VAT-FDH

Author(s): Alexandrina Nastasa, MD,pHD

Co-Author(s):

Luminita Craitoiu-Nirlu, MD

Corneliu Iorgulescu, MD,pHD

Dan Andrei Radu, MD, pHD

Stefan Petre, Ing.

Viviana Gondos, Ing

Stefan Bogdan, MD,pHD

Radu Gabriel Vatasescu, MD,pHD

Elias Universitary Emergency Hospital, Bucharest

Blv. Marasti nr 17, sector 1 Bucuresti

\section{Introduction | Objectives:}

Cateter ablation (CA) for atrial fibrillation (AF) has been proven to be extremely effective in maintaining sinus rhythm but success rates still vary widely. Procedural costs are not negligible and a low, but nonetheless present, rate of potentially severe complications exists, therefore an accurate estimation of results is necessary 
in order to assess the risk-benefit ratio. Several studies have proposed different scores for predicting postprocedural success, such as APPLE, CAAP-AF, MB-LATER, SUCCESS, but most have not been validated yet in external cohorts, hence further research for the optimal formula is justified.

Our objective was to find independent predictors of recurrence after CA for AF and group them into a new, easy to calculate composite score.

Methods: A total of 263 consecutive patients with AF who underwent CA in our centre were retrospectively analyzed. Variables that were significantly associated with recurrence risk according to univariate analysis were introduced in a Cox multivariate regression. The independent predictors of recurrence were used to create a new predicting score, after empirically assigning them different weights.

Results: The mean follow-up period was $37.6 \pm 23.4$ months. The type of AF (persistent), f-waves < $0.1 \mathrm{mV}$, indexed LA volume, the presence of type II diabetes and smaller height were found to be independent predictors of recurrence and were used to create the new scoring model, VAT-DHF. The ROC curve for this new score showed an AUC 0.869, $\mathrm{p}<0.0001,95 \% \mathrm{CI}[0.802-0.936]$ while those for APPLE and $\mathrm{CHA}_{2} \mathrm{DS}_{2}$ Vasc showed AUC 0.765, 95\% CI [0,637-0.893] and respectively, AUC 0.655,95\%CI [0,580-0.730]. Patients who had a VAT-DHF score between 0-3.25 had a rate of success of $95.7 \%$, those with a score between 3.25-6 of $76.3 \%$, and those with a score [?] 6 had a rate of success of $25 \%(\mathrm{p}<0.0001)$. A cut-off value of 5,75 gives the score a sensitivity of $87 \%$ and a specificity of $75 \%$.

Conclusions: The novel and simple VAT-DHF score is superior to the APPLE and $\mathrm{CHA}_{2} \mathrm{DS}_{2}$-VASc scores in predicting outcomes after catheter ablation for AF. It may provide a useful clinical tool to identify patients with low, intermediate or high risk of AF recurrence, but further validation is necessary.

AFS2022-19Submission Number: 1167508

Abstract Title: Mechanism and Outcomes of Atrial Tachycardia Ablation in Patients with Mitral Valve Surgery

Author(s): Hikmet Yorgun, Prof. Dr.

Co-Author(s):

Gul Sinem Kılıç, Dr.

Burak Sezenöz, Assoc. Prof.

Kudret Aytemir, Prof. Dr.

Hacettepe University

Hacettepe University, Faculty of Medicine, Department of Cardiology, Ankara, Turkey

Introduction | Objectives:

Atrial tachycardia (AT) is a common rhythm disorder after mitral valve surgery which may comprise both atria. We aimed to evaluate the ablation outcomes of AT in patients with previous mitral valve (MV) surgery.

\section{Methods:}

A total of 60 symptomatic patients with AT were enrolled. Activation mapping was performed during tachycardia in all patients as well as entrainment mapping when needed. Follow-up data also recorded to determine recurrences.

\section{Results:}

In 79 procedures, a total of $101 \mathrm{ATs}$ were documented [35 (34.6\%) ATs were right-sided, 65 (64.3\%) ATs were left-sided and $1(0.9 \%)$ AT was biatrial]. Mean number of ATs per procedure was $1.4 \pm 0.6$. Macroreentry was the primary mechanism in right and left atrium (97\% and $66.1 \%$, respectively). The mean follow-up 
duration was $33.4 \pm 17.7$ months. Freedom from ATa after index procedure was $78.3 \%$ at 12 months and $65.9 \%$ at 24 months. After multiple procedures, freedom from ATa was 80\%, during the follow-up.

\section{Conclusions:}

Left ATs are more common compared to the right ATs and macroreentry is the major mechanism in both right- \& left-sided ATs. RF ablation of ATs had favourable long-term outcomes in patients with MV surgery.

\section{AFS2022-19}

\section{Uploaded File(s)}

Mechanism and Outcomes of Atrial Tachycardia Ablation in Patients with Mitral Valve Surgery
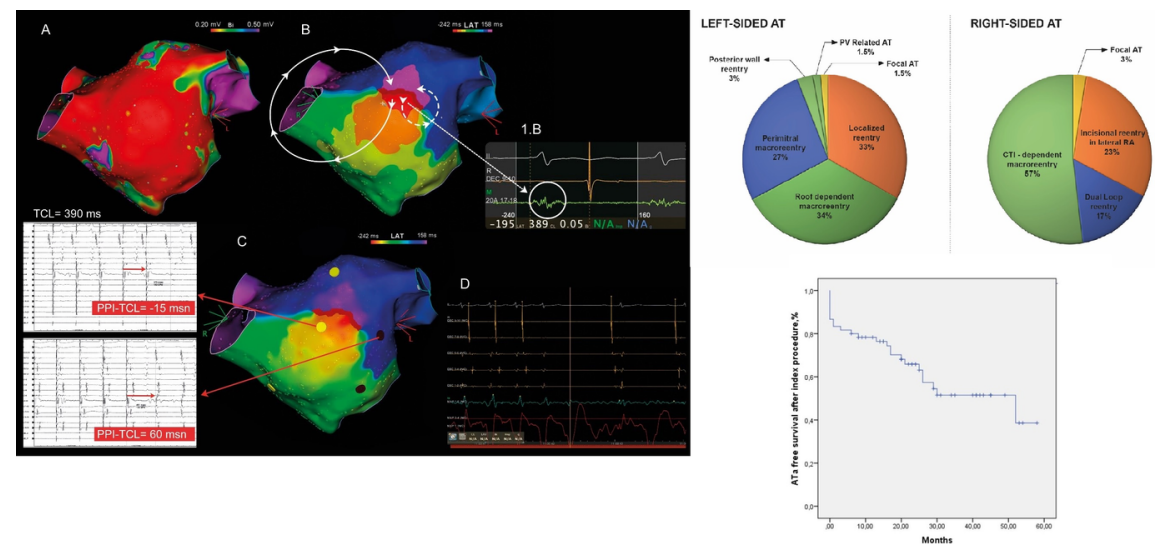

\section{AFS2022-20 Submission Number: 1167736}

Abstract Title: Scar distribution and tachycardia mechanisms in left sided atrial tachycardia after mitral valve surgery.

Author(s): Hikmet Yorgun, Prof. Dr.

Co-Author(s):

Sinem Gül Kılıç, Dr.

Burak Sezenöz, Assoc. Prof.

Kudret Aytemir, Prof. Dr.

Hacettepe University

Hacettepe University, Faculty of Medicine, Department of Cardiology, Ankara, Turkey

Introduction | Objectives:

Atrial tachycardia (AT) is a common rhythm disorder after mitral valve surgery and scar related reentry is one the most important mechanism in these patients. We aimed to evaluate the left atrial scar distrubution and tachycardia features in patients with previous mitral valve (MV) surgery undergoing left-sided AT ablation.

\section{Methods:}

A total of 36 symptomatic patients with AT were enrolled. Initially left atrial voltage mapping was performed to determine left atrial low voltage areas and subsequent activation mapping of clinical tachycardias was 
done in all patients as well as entrainment mapping when needed. Follow-up data also recorded to determine recurrences.

\section{Results:}

In 36 patients, a total of 50 left-sided ATs were mapped. Mean number of ATs per procedure was $1.5 \pm 0.8$. Anterior wall $[53.2 \%(17.9 \%-74.6 \%)]$, septum $[53.7 \%(34.7 \%-80.0 \%)]$ and roof $[40.1 \%(21.3 \%-72.5 \%)]$ were the left atrial sites with highest ratio of low voltage areas. Roof dependent flutter $[17(34 \%)]$ and perimitral flutter $[15(30 \%)$ ] was the most common tachycardia mechanism. 35 (70\%) ATs were defined as macroreentry, $10(10 \%)$ ATs were defined as localized reentry in our population. Concomitant typical atrial flutter ablation was performed in 4 patients. The mean follow-up duration was $31.2 \pm 16.3$ months. Reccurence occured in $13(36.1 \%)$ patients during follow-up. Left-sided ATs were the tachycardia localization in 8 patients and 1 patient had a typical atrial flutter who underwent a re-do procedure. Atrial fibrillation was detected in 2 patients.

\section{Conclusions:}

Left atrial low voltage areas are responsible for left-sided ATs after MV surgery. Anterior wal, septum and roof were the most common sites for scar localization. Scar-related reentry is an important tcahycardia mechanism in these patients.

\section{AFS2022-20}

\section{Uploaded File(s)}

Scar distribution and tachycardia mechanisms in left sided atrial tachycardia after mitral valve surgery.
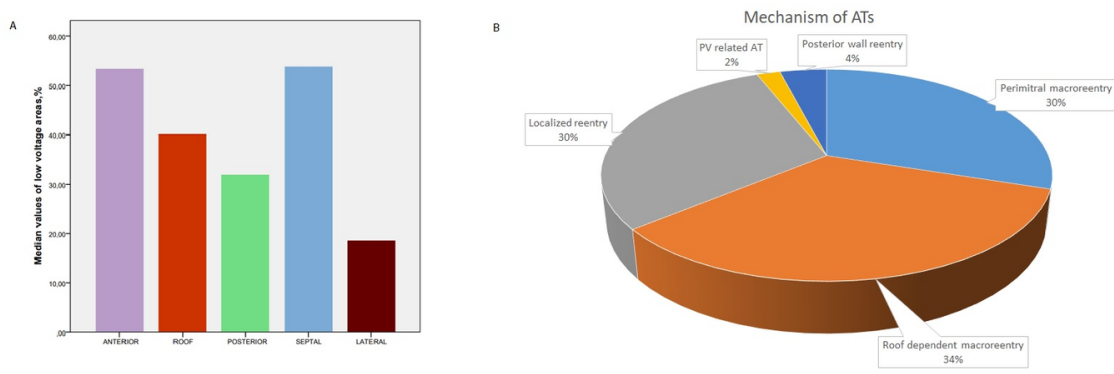

\section{AFS2022-21Submission Number: 1167768}

Abstract Title: Adverse Events And Modes Of Failure Of Arctic Front Catheter: Insights From The FDA Maude Database

Author(s): Ramez Morcos, MD, MBA

\section{Co-Author(s):}

Mohamed Hamed, MD - Florida Atlantic University

Charl Khalil, MD - John H. Stroger hospital of cook county

Haider Al Taii, MD - University of Oklahoma Health Sciences

Brian Blair, BS - Charles E. Schmidt College of Medicine, Florida Atlantic University

Daniel Jacobs, BS - Charles E. Schmidt College of Medicine, Florida Atlantic University

Mina Fares, MD - Oxford University Hospitals NHS Foundation Trust, Department of Cardiology, Headington, Oxford. 
Martin Kloosterman, MD - FLorida Atlantic University, Cardiac Arrhythmia Service

Jonathan Rosman, MD - Florida Atlantic University, Cardiac arrhythemia services

Jose Torres, MD - Florida Atlantic University

Luis Mora, MD - Florida Atlantic University, The Arrhythmia Center of South Florida

Florida Atlantic university

$\mathrm{N} / \mathrm{A}$

\section{Introduction | Objectives:}

Background: Data on the mechanisms of failure of Cryoballoon catheter (Arctic front) are limited. Objectives: To assess the reported mechanisms of failure, complications, and clinical consequences of Arctic front in the United States.

\section{Methods:}

We queried the Food and Drug Administration (FDA) Manufacturer and User Facility Device Experience (MAUDE) database between January 2011 and January 2021 for reports on Arctic front (Medtronic, Inc, Minneapolis, MN). The primary outcome was the mechanism of failure. Secondary outcomes included clinical consequences of device failure.

\section{Results:}

The final analysis included 572 reports of failure/complication (Arctic front $\mathrm{n}=193 \&$ Arctic front advance $\mathrm{n}=379)$. The most commonly reported failure mode was Kink of the catheter (43.5\%) overall (28.5\% with Arctic front and $51.2 \%$ with Arctic front advance), located mainly in the guidewire (90\%) overall (28.5\% with Arctic front and $51.2 \%$ with Arctic front advance). Fracture of catheter component (i.e., guidewire, pebax, Balloon connector, shaft, tip, or bowden) was (30.8\%) overall (18.1\% with Arctic front and $37.2 \%$ with Arctic front advance) mainly in the guidewire (94.3\%) overall (97.1\% with Arctic front and 93.6\% with Arctic front advance). The safety system detected fluid in the catheter and stopped the injection $(24.3 \%)$ overall (18.7\% with Arctic front and $27.2 \%$ with Arctic front advance). Air embolism was the most common complication $(2.6 \%)$, followed by hemodynamic collapse (1\%). Cryoballoon was replaced $(26.2 \%)$. While the procedure was aborted in $(2.6 \%)$ of the reports, no death or surgical conversion occurred.

\section{Conclusions:}

Guidewire kink and fracture were the most common failure mode, and air embolism was the most common complication reported with Arctic front. Future efforts should focus on an innovative guidewire structure.

AFS2022-22Submission Number: 1168079

Abstract Title: Adverse Events And Modes Of Failure Of Thermocool Smart Touch Catheter: Insights From The FDA Maude Database

Author(s): Ramez Morcos, MD, MBA

Co-Author(s):

Mohamed Hamed, MD - Florida Atlantic University

Charl Khalil, MD - John H. Stroger Hospital of Cook county

Haider Al Taii, MD - University of Oklahoma Health Sciences Center

Daniel Jacobs, BS - Charles E. Schmidt College of Medicine, Florida Atlantic University

Brian Blair, BS - Charles E. Schmidt College of Medicine, Florida Atlantic University 
Mina Fares, MD - Oxford University Hospitals NHS Foundation Trust, Department of Cardiology, Headington, Oxford.

Mohamed Abdelazeem, MD - St. Elizabeth's Medical Center, Brighton, Massachusetts

Mora Luis, MD - Florida Atlantic University,The Arrhythmia Center of South Florida.

Vivas Yoel, MD - Florida Atlantic University,The Arrhythmia Center of South Florida.

Florida Atlantic university

$\mathrm{N} / \mathrm{A}$

Introduction | Objectives:

Background: Data on the mechanisms of failure of Radiofrequency catheter (Thermocool@) are limited.

Objectives : To assess the reported mechanisms of failure, complications, and clinical consequences of Thermocool@ Smart Touch catheter in the United States.

Methods:

We queried the Food and Drug Administration (FDA) Manufacturer and User Facility Device Experience (MAUDE) database between January 2020 and December 2021 for reports on Thermocool@ Smart Touch (Biosense Webster, Inc, Irvine, CA). The primary outcome was the mechanism of failure. Secondary outcomes included clinical consequences of device failure.

\section{Results:}

The final analysis included 468 reports of failure/complication (Unidirectional $n=89 \&$ Bidirectional $n=379$ ). The most commonly reported failure mode was Fracture of catheter component (i.e., guidewire, pebax, Balloon connector, shaft, tip, or bowden) was $(17.7 \%)$ overall $(33.7 \%$ with Unidirectional and $14 \%$ with Bidirectional) mainly in the pebax (95.5\%) overall (90\% with Unidirectional and $90.6 \%$ with Bidirectional). Part of the Catheter's internal part was unraveled in (14.3\%) overall $(22.5 \%$ with Unidirectional and $12.4 \%$ with Bidirectional). Cardiac tamponade was the most common complication $(37.2 \%)$ overall $(24.7 \%$ with Unidirectional and $40.1 \%$ with Bidirectional), followed by hemodynamic collapse $(23.1 \%)$ overall (14.6\% with Unidirectional and $25.1 \%$ with Bidirectional). Pericardiocentesis was performed in $(46.6 \%)$ overall $(31.5 \%$ with Unidirectional and $50.1 \%$ with Bidirectional). The procedure was aborted in (18.5\%) overall $(17.9 \%$ with Unidirectional and $2.6 \%$ with Bidirectional) with the need of surgical conversion in $(12.6 \%)$ overall (11.2\% with Unidirectional and $12.9 \%$ with Bidirectional). Death occurred in $(4.3 \%)$ overall (4.5\% with Unidirectional and $4.2 \%$ with Bidirectional).

\section{Conclusions:}

Fracture of catheter mainly at the pebax and internal part unravel were the most common failure mode. Cardiac tamponade was the most common complication reported. Further improvements in catheter tip are needed to optimize deliverability and minimize the risk for tamponade.

\section{AFS2022-23 Submission Number: 1168039}

Abstract Title: Switching Indwelling Foley catheter to External Catheter for Outpatient Atrial Fibrillation Ablation: A quality improvement Project

Author(s): Murtaza Sundhu, MD

\section{Co-Author(s):}

Christopher Reggio, DO - Tower Health - Reading Hospital

Usama Nasir, MD - Tower Health - Reading Hospital

Tayyab ali Waheed, MD - Tower Health - Reading Hospital 
Muhammad Ameen, MD - Tower Health - Reading Hospital

Arun Kundra, MD - Tower Health - Reading Hospital

Tower Health - Reading Hospital

420 S 5th Ave, West Reading, PA 19611

Introduction | Objectives: Outpatient atrial fibrillation ablation is the most frequent ablation in the $\mathrm{EP}$ lab, and it is done under general anaesthesia and all the patients get indwelling foley catheter. Most of the patients are discharged the same day but some patients get admitted and the reason at times include urinary tract infections, urinary retention, or haematuria. It is not known if using an external catheter is safe for these patients. The goal of this study was to evaluate if replacing indwelling catheter with external catheter is safe and how it effects the number of patients requiring overnight admission to the hospital after outpatient atrial fibrillation ablation.

Methods: Collaboration was done with the Anesthesia department and indwelling Foley catheter were replaced with external catheter at the beginning of May 2021. Data was collected for 7 months before and after the switch. All morning ablations by only one operator were included to reduce confounding of interpersonal variability and afternoon cases. Ablations for ventricular tachycardia, accessory pathway and atrial flutter alone were excluded. Outpatient atrial fibrillation ablations with or without flutter ablation were included.

Results: Total of 41 patients were included in the study. Mean age was 65.9 years, $78 \%$ were males (32/41), 27\% were diabetic (11/41),68\% had hypertension $(28 / 41), 24 \%$ had coronary artery disease $(10 / 41)$ and $29 \%$ had congestive heart failure (12/41) at baseline. Indwelling foley catheter was placed in $39 \%(16 / 41)$ patients and external catheter in $61 \%(25 / 41)$ patients. Percentage of patients admitted to hospital for overnight admission dropped from 19\% (3/16) with the indwelling foley catheter to $8 \%$ $(2 / 25)$ with the external catheter placement. There were no complications by using the external catheter system and the admissions were done for monitoring / bleeding from the access site.

Conclusions: External urinary catheter system can be safely implemented for outpatient atrial fibrillation ablation. We also noted a significant numerical reduction in the patients admitted to the hospital for observation. More studies are needed for generalizability of these findings.

\section{AFS2022-23}

\section{Uploaded File(s)}

Outcomes of indwelling Foley catheter versus external catheter on Outpatient Atrial Fibrillation Ablation 

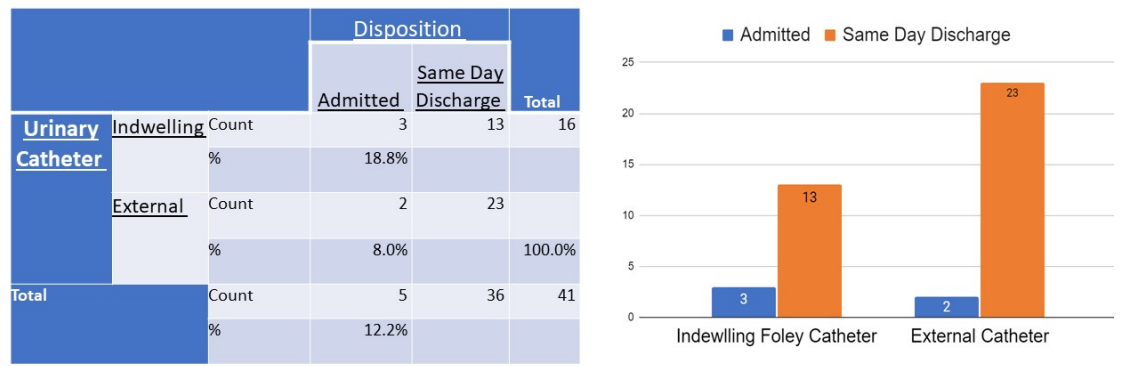

AFS2022-24Submission Number: 1168169

Abstract Title: Persistent Atrial Fibrillation Ablation: First Experience with Multipolar Grid-Shaped Mapping Catheter and Omnipolar Technology.

Author(s): Sergio Conti, MD, $\mathrm{PhD}$

Co-Author(s):

Giuseppe Sgarito, MD, PhD - Cardiac Electrophysiologist, ARNAS Civico Hospital

ARNAS Civico Hospital

Piazza Nicola Leotta 4, 90127 - Palermo, Italy

Introduction | Objectives:

Substrate mapping during AF with traditional bipolar voltages may be challenging because of AF's complex mechanism. The presence of wave collisions and fractionation results in low bipolar voltage, worsened by the orientation-dependence of bipolar EGMs. Omnipolar EGMs are bipolar signals along specific directions obtained by a grid-shaped multielectrode catheter and signal processing to generate maps in real-time from single depolarizations. Omnipolar technology (OT) provides voltage, timing, and activation direction assessments independent of catheter orientation by incorporating simultaneously acquired EGMs from multiple directions. AF OT-based mapping may better evaluate the complex and time-varying AF mechanisms, such as wave collisions and fractionations.

Methods: n/a

\section{Results:}

A 69-year-old woman with drug-refractory symptomatic persistent AF underwent catheter ablation. The procedure was performed using the EnSite X mapping system, HD Grid SE, and the OT (Abbott). A baseline substrate map was performed during AF with and without the OT (Figure 1A - B). OT map showed a continuous fragmented activation on the posterior roof adjacent to the LSPV. AF omnipole-based vector fields have significant spatial and temporal disorganization (Figure 1C). PVI was performed using a sensor-enabled ablation catheter. While ablating in this region, AF converted to sinus rhythm (green dot) (Figure 1D). The roofline was completed, and pacing maneuvers were performed to confirm bidirectional block across the line, also confirmed with the LiveView software.

\section{Conclusions:}


High-definition multipolar mapping using the HD Grid can be rapidly and accurately performed during complex atrial arrhythmias. In particular, OT provides a rapid, high-density, orientation-independent assessment of the underlying substrate. To the best of our knowledge, this is the first demonstration of how the OT may improve the mapping of complex substrate and arrhythmia such as persistent AF. Although it is still controversial whether ablation of fractionated electrograms during AF improves the acute and long-term outcome, it is unquestionable that a better substrate definition is at the base of a more tailored procedural strategy.

\section{AFS2022-24}

\section{Uploaded File(s)}

Figure 1. Panel A: Substrate map during AF using the Omnipolar Technology (OT); Panel B: Substrate map during AF without OT; Panel C: Activation map using the OT. Multiple wave collisions are evident close of the green dot (restoration of sinus rhythm); Panel D: Restoration of sinus rhythm during ablation on the area of maximum wave collisions and fractionations.

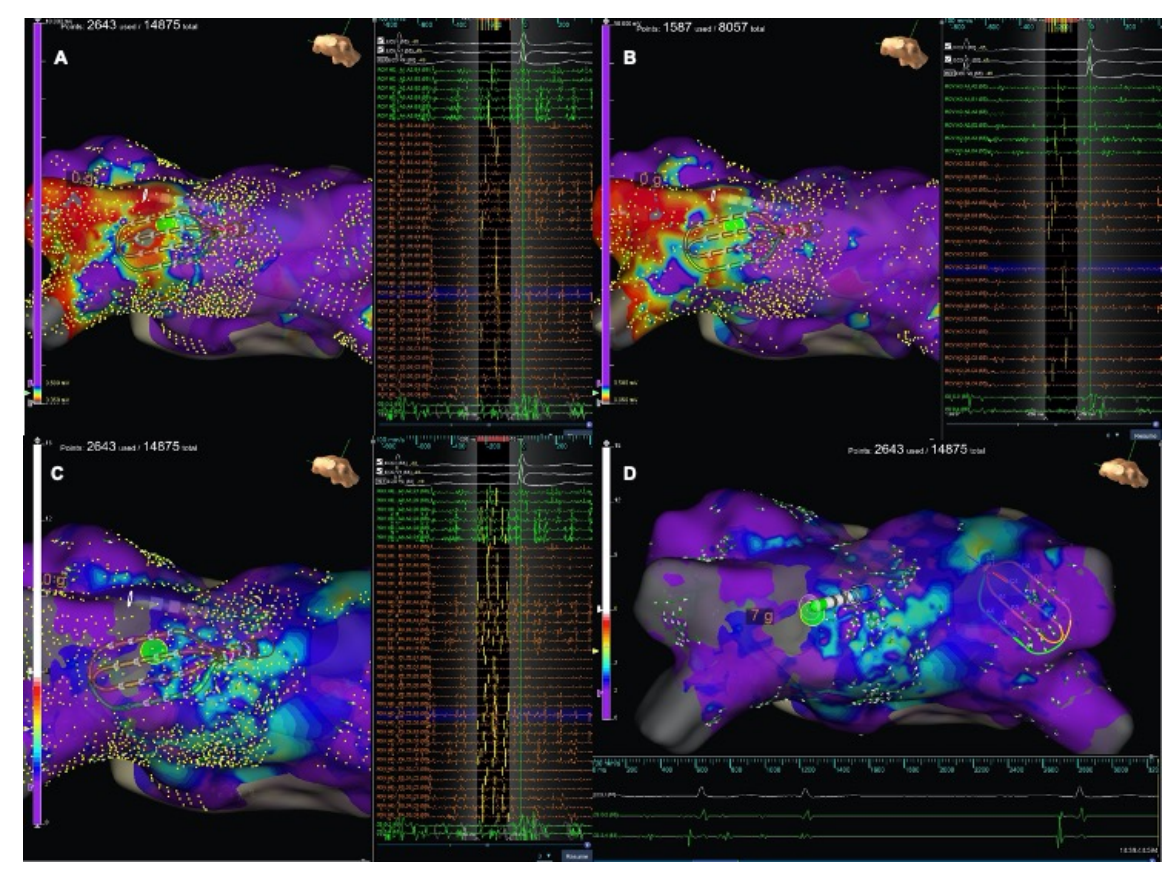

\section{AFS2022-25Submission Number: 1168207}

Abstract Title: Persistence of posterior wall isolation guided by ablation index evaluated during a redo.

Author(s): Sergio Conti, MD, $\mathrm{PhD}$

Co-Author(s):

Giuseppe Sgarito, MD, PhD - Cardiac Electrophysiology, ARNAS Civico Hospital

ARNAS Civico Hospital

Piazza Nicola Leotta 4, 90127 - Palermo, Italy

Introduction | Objectives:

The posterior wall of the left atrium (LA) is an arrhythmogenic substrate responsible for initiating and maintaining atrial fibrillation $(\mathrm{AF})$. In addition to pulmonary vein isolation (PVI), posterior wall isolation 
(PWI) aims to eliminate AF triggers and drivers. However, ablation at the posterior wall of the LA using contiguous and optimized radiofrequency lesions remains challenging as continuous and effective lesion sets are necessary. Moreover, there are few data regarding the impact of the Ablation Index (AI) on the efficacy of PWI. This study aims to evaluate the effects of AI-guided PWI in patients with persistent AF undergoing PVI plus PWI.

\section{Methods:}

Twenty consecutive patients with symptomatic drug-refractory persistent AF underwent PVI plus PWI by linear lesions across the roof and bottom. The procedures were performed using the CARTO mapping system, SmartTouch SF ablation catheter, and the LASSO or PentaRay mapping catheter (Biosense Webster). AI settings were 500-550 for the anterior aspect of the PVs and the roofline, while 450-500 for the posterior aspect of the PVs and the bottom line and/or PW lesions. After the blanking period, 4/20 patients underwent redo because of atrial arrhythmia ( 3 atrial flutter - AFL and 1 atrial tachycardia - AT). In addition, a complete LA map was performed in these patients to evaluate for PV and PW reconnections. In the redo, the PentaRay mapping catheter was used for the activation and substrate map.

Results: Sixteen patients had no recurrence documented during the follow-up. After a 90-days blanking period, three patients underwent redo; two patients because of right AFL ablation, and one patient left AT. Two out of three were cavotricuspid isthmus dependent counterclockwise AFL, and the third was intercaval right AFL. The AT was mapped at the base of LAA. All these arrhythmias were successfully ablated. Of great interest, in all the redo, the LA substrate map performed with CARTO confirmed the persistence of PVI and PWI.

\section{Conclusions:}

PWI guided by AI seems to be an effective approach. During the LA map performed at the redo, the PW ablated at the index procedure with the AI guidance remains isolated after the 90-days blanking period suggesting that AI improved lesion formation on the LA posterior wall.

AFS2022-25

Uploaded File(s)

Persistence of posterior wall isolation. 


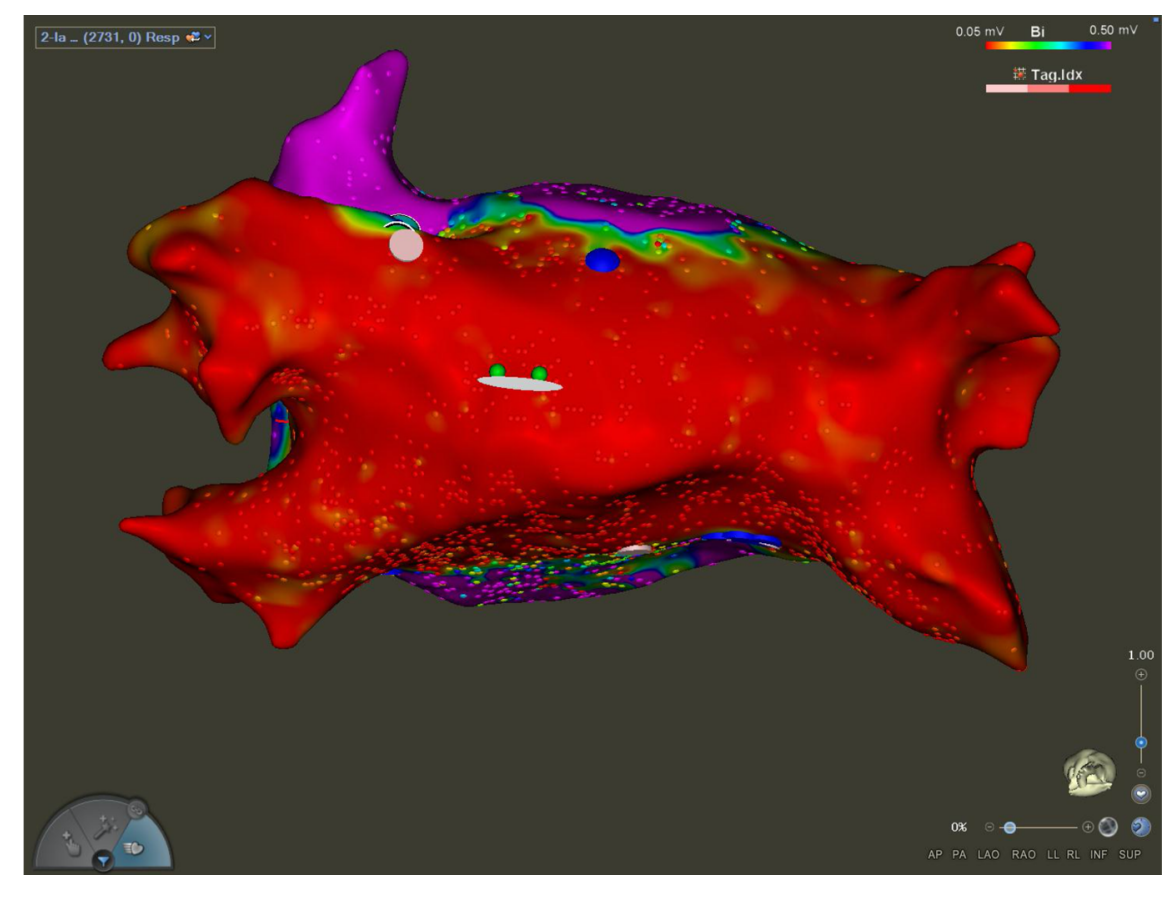

AFS2022-26 Submission Number: 1168943

Abstract Title: Intracardiac Echocardiography May Improve Procedural Safety in Paroxysmal Atrial Fibrillation Catheter Ablations

Author(s): Jose Osorio, MD

Co-Author(s):

George Monir, MD

Daniel Melby, MD

Paul Tabereaux, MD

Andrea Natale, MD

Harish Manyam, MD

Charles Athill, MD

Craig Delaughter, MD

Anshul Patel, MD

Philip Gentlesk, MD

Christopher Liu, MD

Jeffrey Arkles, MD

Hugh Thomas McElderry, MD

Luigi Di Biase, MD, PHD

Grandview Medical Center Alabama Cardiovascular Group

3686 Grandview Pkwy \#720, Birmingham, AL 35243 
Introduction | Objectives: Intracardiac echography (ICE) is widely used in atrial fibrillation ablation procedures for left atrial appendage (LAA) thrombus detection, esophageal monitoring, and catheter visualization during the procedures to guide transseptal puncture or catheter positioning. Altogether, utilization of ICE may reduce fluoroscopy use during the procedure. However, there exists a paucity of sources supporting the safety benefits of using ICE. This study aims to evaluate the procedural safety and effectiveness of using ICE vs. no ICE in PAF ablation procedures.

Methods: The Surpoint post-approval study was a prospective, multicenter, nonrandomized study conducted at 30 US sites. The ablation procedure was performed with a porous tip contact force catheter and tag index. The primary safety endpoint was primary adverse event (PAE) rate occurring within 7 days post ablation. The primary effectiveness endpoint included $12 \mathrm{M}$ freedom from atrial arrhythmia recurrences and 5 additional failure modes. Fisher's exact test sub-analysis of the primary safety and effectiveness endpoints were performed in patient cohorts with or without ICE utilization during the procedure, as well as by fluoroscopy time ( $<1 \mathrm{~min}$ vs [?] $1 \mathrm{~min})$.

Results: Of 283 patients enrolled, 261 had the catheter inserted and underwent ablation (safety population). The PAE rate in ablation procedures where ICE was used was about 3.7-fold lower in comparison to those without ICE usage $(3.5 \%$ [8/232] vs $13.0 \%$ [3/23], $\mathrm{p}=0.0656)$. The decrease was most substantial for cardiac tamponade/perforation and pericarditis events (ICE vs. no ICE: $0.9 \%$ vs. $4.3 \% ; 0.9 \%$ vs. $8.7 \%$, respectively). Primary effectiveness was comparable for both groups $(76.4 \%$ [159/208] vs $70.0 \%$ [14/20], p=0.58). There were no significant differences of PAE rates and effectiveness success rates between procedures performed with extremely low fluoroscopy of $<1 \mathrm{~min}$ and those of [?] $1 \mathrm{~min}$ (safety: $4.85 \%$ vs $3.45 \%, \mathrm{p}=0.74$; effectiveness: $77.3 \%$ vs $85.9 \%, \mathrm{p}=0.11$ ).

Conclusions: In addition to facilitating fluoroscopy reduction, the use of ICE in paroxysmal AF ablation procedures is associated with a possible benefit of about 3.7-fold reduction in primary safety rate.

AFS2022-27Submission Number: 1172741

Abstract Title: Clinical Outcomes of Ablation for Persistent Atrial Fibrillation with Minimal Fluoroscopy

Author(s): Jose Osorio, MD

Co-Author(s):

Gustavo Morales, MD

Anil Rajendra, MD

Tina Hunter, $\mathrm{PhD}$

Alabama Cardiovascular Group

3686 Grandview Parkway Suite 720 Birmingham, AL 35243

Introduction | Objectives: Recent advances in electroanatomical mapping, intracardiac echocardiography, and sheath technology have enabled catheter ablation workflows that reduce or eliminate fluoroscopy use without compromising safety or efficacy outcomes. Limited real-world evidence exists on minimal fluoroscopy ablation specific to persistent atrial fibrillation (PsAF) populations. This study aimed to evaluate the safety, procedural efficiency, and effectiveness of a minimal-fluoroscopy workflow, utilizing a contact force ablation catheter and stability software, in a real-world PsAF population.

Methods: This was a non-randomized single-center retrospective cohort study. All procedures were performed at a single high-volume site by three operators. Patients were evaluated and treated per standard of care and data was collected prospectively per standardized case report forms. All consecutive de novo ablations of symptomatic PsAF in adults [?]18 years between 2017-2018 with a contact force radiofrequency catheter were included, with long-standing PsAF ( $>1$ year) cases excluded. Pulmonary veins (PV) were 
isolated by wide area circumferential ablation using catheter stability software. Contact force and impedance were monitored in real time. Patients were followed for 12 months for complications and clinical effectiveness.

Results: Of 406 ablations meeting all inclusion criteria, average age was $67.8 \pm 10.1$ years, $65.3 \%$ male, CHA2DS2-Vasc Score was $2.9 \pm 1.5$. Majority of procedures (85.4\%) were performed without fluoroscopy and those using fluoroscopy averaged only $0.6 \pm 1.5 \mathrm{~min}$. Most procedures $(97.0 \%)$ included ablation beyond PV, with $55.7 \%$ including both cavotricuspid isthmus ablation and substrate modification. Serious complications were few (2.0\%). Procedure times averaged $89.5 \pm 34.6 \mathrm{~min}$. with $677 \pm 232 \mathrm{~mL}$ of fluid delivered via ablation catheter. At 12 months follow up, $73.6 \%$ [68.7\%, 77.8\%] of patients were free from post-blanking ( $>90$ days) recurrence and reablation rate was $13.1 \%$.

Conclusions: Despite more extensive ablation than a typical paroxysmal AF population, ablation with minimal fluoroscopy can be performed safely and without compromise to procedural efficiencies or clinical effectiveness in PsAF patients.

\section{AFS2022-28Submission Number: 1169077}

Abstract Title: Dynamic mapping and identification of non-PV targets for ablation in patients with atrial fibrillation

Author(s): Paul Garabelli, MD

Co-Author(s):

Jose Martel, MD, MPH, FHRS

Kamel Addo, MD

Kent Nilsson, MD

Saumil Shah, MD, FHRS

Toshimasa Okabe, MD

Jorge Diaz, MD, FACC, FHRS

Henry Huang, MD

Caroline Tao, $\mathrm{PhD}$

Coty Jewell, MD

Oklahoma Heart Hospital South

5224 E Interstate 240 Service Rd, Oklahoma City, OK 73135

Introduction | Objectives: Ablation of non-pulmonary vein (PV) triggers is recognized as an important step to improve outcomes in atrial fibrillation (AF) ablation. However, non-PV foci are sometimes difficult to identify and eliminate.

\section{Methods:}

Procedural data from a total of 503 patients undergoing de novoablation for atrial fibrillation were prospectively collected. A high-density grid-style mapping catheter (HD Grid) was used either with or without dynamic mapping (LiveView). PV isolation (PVI) was successfully conducted in all patients. Additional ablations were determined at the physician's discretion. Acute procedure data such as RF time, procedure time, and acute outcomes were compared between cases performed with and without dynamic mapping.

\section{Results:}

Both paroxysmal and persistent AF were included in the dataset (PAF:313, PersAF:190). Among 503 patients, LiveView was used in 147 cases [PAF: 85/313 (27.2\%), PersAF: 62/190(32.6\%)]. A higher proportion 
of the PAF cases utilized a PVI only approach [PAF: 158/313 (50.5\%), PersAF: 63/190 (33.2\%), P< 0.05]. The frequency of ablating non-PV targets was $51.1 \%(182 / 356)$ and $61.9 \%$ (91/147) when HD Grid was used without and with LiveView, respectively $(\mathrm{p}<0.05)$. The most common non-PV ablation reported were cavotricuspid isthmus (CTI) line, roofline, and posterior wall isolation (HD Grid: 28\%, 27.5\%, and 14\%; HD Grid+LiveView: $49.7 \%, 10.9 \%$, and $28.6 \%$, respectively). In PersAF, an average of 1.28 and 1.42 non-PV targets were ablated when HD Grid was used without and with LiveView. Despite more non-PV targets being ablated when LiveView was used in PersAF, the total RF time was significantly shorter (HD Grid: 37.2 $\pm 24.1 \mathrm{~min}$; HD Grid+LiveView: 29.1 $\pm 16.4 \mathrm{~min}, \mathrm{P}<0.05$ ) (Table 1).

Conclusions: Ablation of non-PV targets was more common in patients with PersAF. The use of realtime regional dynamic mapping was also associated with reduction in RF duration, especially in PersAF, potentially attributed to better visualization and more precise identification of substrate. While trends of improved procedure efficiency were observed, further controlled study may be warranted to confirm this observation.

\section{AFS2022-28}

\section{Uploaded File(s)}

Table 1: Comparison of procedure characteristics from cases collected without and with LiveView Dynamic Display in PAF and PersAF cases.

\begin{tabular}{|l|l|l|l|l|l|}
\hline & \multicolumn{2}{|l|}{ Paroxysmal AF } & \multicolumn{2}{l|}{ Persistent AF } \\
\cline { 2 - 6 } & HD Grid & $\begin{array}{l}\text { HD } \\
\text { Grid+LiveView }\end{array}$ & HD Grid & $\begin{array}{l}\text { HD } \\
\text { Grid+LiveView }\end{array}$ \\
\hline Number of cases & 228 & 85 & 128 & 62 \\
\hline RF Time(minutes) & $25.6 \pm 24.8$ & $23.6 \pm 19.4$ & $37.2 \pm 24.1$ & $29.1 \pm 16.4^{*}$ \\
\hline Fluoro Time (minutes) & $5.8 \pm 9.5$ & $4.0 \pm 6.9$ & $9.6 \pm 13.7$ & $7.3 \pm 8.3$ \\
\hline Procedure time (minutes) & $135.8 \pm 91.5$ & $125.6 \pm 56.1$ & $145.4 \pm 85.8$ & $148.5 \pm 63.7$ \\
\hline
\end{tabular}

${ }^{*} \mathrm{P}<0.05$

\section{AFS2022-29Submission Number: 1169082}

Abstract Title: Utilization of real-time dynamic mapping in redo paroxysmal atrial fibrillation procedures: multicenter U.S. experience

Author(s): Kamel Addo, MD

Co-Author(s):

David DeLurgio, MD

Saumil Shah, MD, FHRS

Gregory Deam, MD

F. Kevin Hackett, MD, FACC

Toshimasa Okabe, MD

Opesamni Esan, MD

Caroline Tao, $\mathrm{PhD}$

Jose Martel, MD, MPH, FHRS

Mount Carmel St. Ann's

477 Cooper Road, Suite 200, Westerville, Ohio 43081 
Introduction | Objectives: Recurrent atrial arrhythmias after catheter ablation for paroxysmal atrial fibrillation (PAF) can be caused by pulmonary vein (PV) reconnections and non-PV triggers. Rapid identification of arrhythmogenic triggers in redo procedures is critical for successful clinical outcomes.

Methods: Acute procedure data from 99 redo PAF cases mapped with a high-density grid-style catheter (HD Grid) were prospectively collected from participating centers in the U.S. Mapping and ablation strategies, including the use of a real-time dynamic mapping data (LiveView), were determined at the physician's discretion. Procedure data such as RF time, procedure time, and acute outcomes were compared between cases performed with and without dynamic mapping.

Results: LiveView was utilized in $25.3 \%$ (25/99) of cases. Non-PV lesions were delivered in 78 cases [HD Grid:59/74 (79.7\%), HD Grid+LiveView: 19/25 (76\%)]. The most common non-PV lesion sets were roofline, cavotricuspid isthmus (CTI) line, and mitral isthmus (MI) line for cases performed without LiveView; posterior wall, MI, and CTI for cases performed with LiveView. The radiofrequency (RF) time, fluoroscopy time, and total procedure time were all shorter in cases utilizing LiveView (HD Grid: 22.3 $\pm 17.6,12.1 \pm 13.3$, and 141.8 \pm 68.7 minutes; HD Grid+Liveview: $16.2 \pm 10.3,5.3 \pm 7.7$, and $128.2 \pm 70.7$ minutes) (Table 1).

\section{Conclusions:}

Compared to cases completed without the use of dynamic mapping, current data suggest trends of improved procedure efficiency and significant reduction in fluoroscopy utilization when high-density mapping was used in combination with real-time dynamic display of regional mapping data in redo PAF procedures. Further controlled studies which examine the long-term outcomes when dynamic mapping data is incorporated into the workflow may be warranted.

AFS2022-29 Uploaded File(s)

Table 1: Comparison of procedure characteristics from cases collected without and with LiveView Dynamic Display in redo PAF cases.

\begin{tabular}{|l|l|l|}
\hline Redo Parosyxmal AF & HD Grid & HD Grid+LiveView \\
\hline Number of cases & 74 & 25 \\
\hline RF Time(minutes) & $22.3 \pm 17.6$ & $16.2 \pm 10.3$ \\
\hline Fluoro Time (minutes) & $12.1 \pm 13.3$ & $5.3 \pm 7.7^{*}$ \\
\hline Procedure time (minutes) & $141.8 \pm 68.7$ & $128.2 \pm 70.7$ \\
\hline * P $<0.05$ &
\end{tabular}

\section{AFS2022-30Submission Number: 1169394}

Abstract Title: Pulmonary vein isolation alone versus pulmonary vein isolation with additional extensive ablation for atrial fibrillation

Author(s): Joey Junarta, MBBS

Co-Author(s):

Naman Upadhyay, MBBS - Thomas Jefferson University Hospital

Andrea Molin, MBBS - Thomas Jefferson University Hospital

Sairamya Bodempudi, MBBS - Thomas Jefferson University Hospital

Sean Dikdan, MBBS - Thomas Jefferson University Hospital

Eric Warner, MBBS - Thomas Jefferson University Hospital

Daniel Joffe, BS - Sidney Kimmel Medical College

Zachary Pang, BS - Sidney Kimmel Medical College 
Daniel Frisch, MD, FACC - Thomas Jefferson University Hospital

Thomas Jefferson University Hospital

Philadelphia, Pennsylvania

Introduction | Objectives: The value of additional ablation beyond pulmonary vein isolation for atrial fibrillation (AF) ablation is unclear, especially for persistent AF. It is uncertain whether substrate modification with additional extensive ablation improves outcomes. We investigate whether pulmonary vein isolation with additional extensive ablation (PVIEA) improves outcomes compared to pulmonary vein isolation alone (PVIA) for AF ablation.

Methods: Consecutive cases of patients with PVIA versus PVIEA were compared between 9/9/2013 to 12/12/2020. Procedural data collected include radiofrequency ablation delivery time (RADT) and arrhythmia inducibility. Clinical data collected include sinus rhythm maintenance post-procedure.

Results: 235 patients were studied (67 PVIA, 168 PVIEA). RADT was shorter when comparing ablation with PVIA versus PVIEA (32 vs $40 \mathrm{~min} ; \mathrm{p}=0.04)$. More arrhythmias were inducible with PVIEA ( $\mathrm{p}<$ 0.01). There was no difference in sinus rhythm maintenance by Kaplan-Meier survival analysis (log rank test $\mathrm{p}=0.75$ ), after 3 or 12 -months between groups overall, and when stratified by AF type (paroxysmal, persistent), left atrial volume, $\mathrm{CHA}_{2} \mathrm{DS}_{2}$-VASc score, left ventricular ejection fraction, or catheter setting (high-power short-duration, standard-power standard-duration, temperature-controlled non-contact-force).

Conclusions: AF ablation with PVIA or PVIEA produces similar sinus rhythm maintenance overall and when stratified by catheter setting and AF type. PVIA reduced procedure times and less arrhythmias were inducible post-ablation.

AFS2022-30

Uploaded File(s)

Kaplan-Meier survival analysis for atrial fibrillation recurrence.

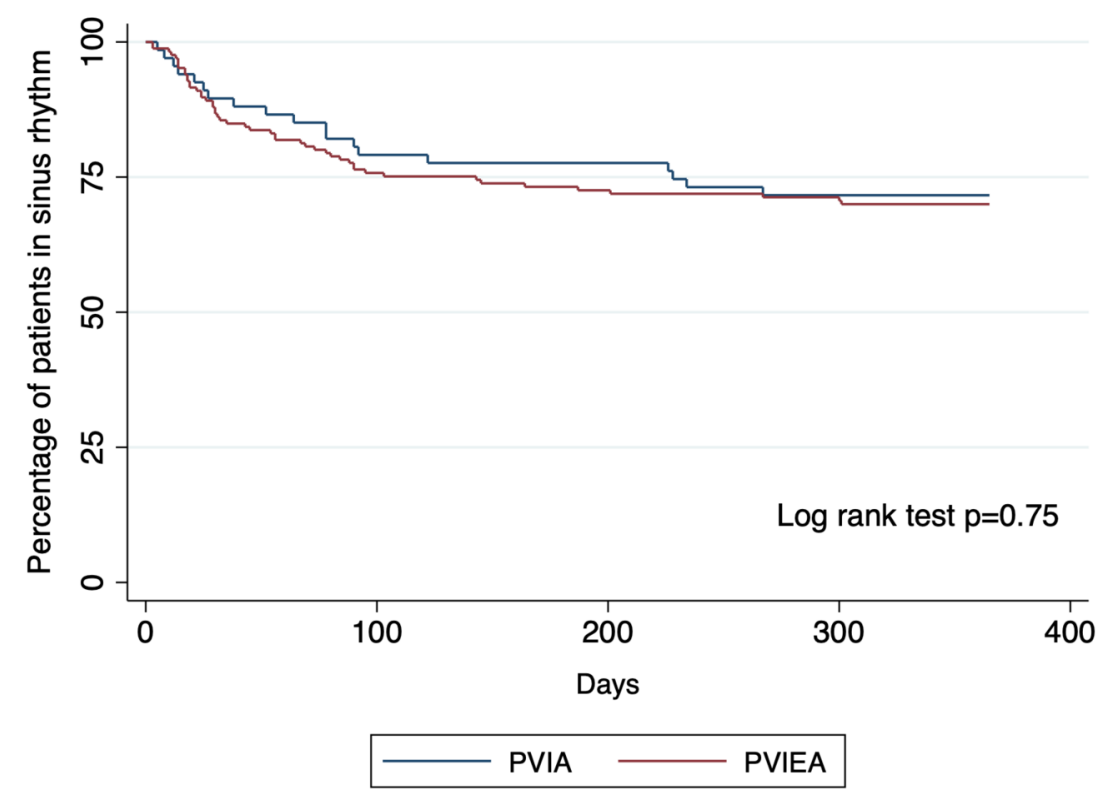

AFS2022-31Submission Number: 1169864 
Abstract Title: Novel Streamlined Technique for Left Atrial Appendage Closure using VersaCross Large Access System

Author(s): Amin Al-Ahmad, MD

Co-Author(s):

Carola Gianni, MD, PHD

Domenico Della Rocca, MD, PHD

Sanghamitra Mohanty, MD

David Tschopp, MD

rodney Horton, MD

Andrea Natale, MD

Texas Cardiac Arrhythmia

3000 IH N 35, Suite 700

\section{Introduction | Objectives:}

Transseptal puncture (TSP) for left atrial (LA) access of large delivery sheaths, such as in left atrial appendage closure (LAAC), typically involves performing TSP with an 8F sheath, then "upsizing" over a wire to the device delivery sheath. This can be especially challenging in fibrotic or aneurysmal septa, and increase the risk of tissue injury and air embolism as a result of multiple device exchanges. This study reports early experience using the VersaCross Large Access (VLA) Solution (Baylis Medical) to streamline and optimize efficiency of LAAC procedures.

\section{Methods:}

Consecutive LAAC procedures performed using the VLA system and the WATCHMAN FLX device (Boston Scientific) were retrospectively evaluated. The VLA system, consists of a $12.5 \mathrm{~F}$ seamless sheath-dilator device, and pigtail RF wire that can be used for vascular access, TSP and WATCHMAN sheath exchange (Fig. 1). The VersaCross pigtail $\mathrm{RF}$ wire $(24 \mathrm{~mm})$ was also used to estimate the left atrial appendage (LAA) size and directly introduce the WATCHMAN sheath into the LAA. Contrast was injected through the WATCHMAN sheath to confirm LAA anatomy and device size. Procedural workflow efficiency was assessed in terms of the time for TSP, WATCHMAN sheath access and implant release, fluoroscopy use and procedural complications.

\section{Results:}

A total of 12 patients underwent LAAC using the WATCHMAN FLX implant and VLA device; $42 \%$ of patients had prior TSP for ablation. TSP and procedure success was $100 \%$ without any intraprocedural complications. Time to TSP was $11.6 \pm 3.3 \mathrm{~min}$ from femoral access, and the $12.5 \mathrm{~F}$ VLA device easily crossed the septum within $0.1 \pm 0.3 \mathrm{~min}$ of TSP. Repeated passage of the seamless VLA device over the pigtail RF wire successfully dilated difficult septa ( $25 \%$ of cases) to confirm patency. Time to WATCHMAN release was $23.3 \pm 3.4 \mathrm{~min}$, and fluoroscopy time and dose were $5.2 \pm 2.3 \mathrm{~min}$ and $85.8 \pm 42.6 \mathrm{mGy}$, respectively.

\section{Conclusions:}

Use of the VersaCross RF wire eliminated unnecessary exchanges and allowed direct access to the LAA to confirm LAA size and optimize WATCHMAN position. The seamless integrated $12.5 \mathrm{~F}$ large access sheathdilator facilitated de novo dilation of the septum for easy exchange of the large bore LAAC delivery system in all patients regardless of history or septal anatomy.

\section{AF2022-31}




\section{Uploaded File(s)}

Novel Streamlined Technique for Left Atrial Appendage Closure using VersaCross Large Access System

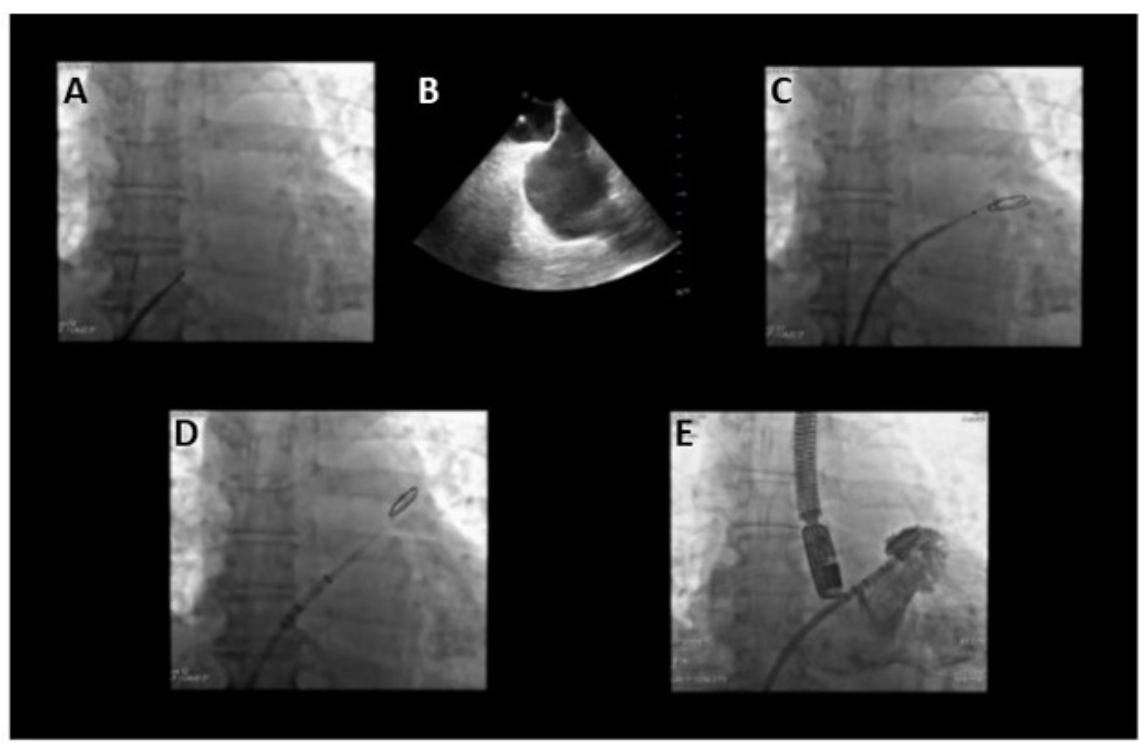

Fig. 1: Images of workflow using VersaCross Large Access Solution (Baylis Medical) for LAAC. Fluoroscopic or transesophageal echocardiographic images of A) RF wire and VLA device in the superior vena cava, B) tenting of septum with RF wire and VLA device, C) Pigtail RF wire in the LAA to approximate appendage size D) direct placement of WATCHMAN delivery sheath in LAA over the RF wire, and E) direct contract injection through WATCHMAN delivery sheath for angiograms

AFS2022-32Submission Number: 1171330

Abstract Title: The Safety of Pharmacological Cardioversion of Recent Onset Atrial Fibrillation with Orally Inhaled Flecainide

Author(s): Christopher Dufton, $\mathrm{PhD}$

Co-Author(s):

Jean Maupas, MS, MD

Jeremy Ruskin, MD

Harry Crijns, MD, PhD

Luiz Belardinelli, MD

InCarda Therapeutics

39899 Balentine Dr Suite 185 Newark, CA, 94560

Background : Acute pharmacological cardioversion (PCV) of recent onset atrial fibrillation (AF) with IV flecainide is generally considered safe for patients who are hemodynamically stable and have no contraindications. We examined data from the Phase 2 open-label study (INSTANT) to evaluate the safety of flecainide acetate oral inhalation solution (FlecIH) for acute PCV of recent onset AF. 
Methods : Safety was evaluated based on peak plasma concentrations of flecainide (Cmax), maximum QRS prolongation ( $\triangle$ QRSmax), cardiovascular events (CVEs) of special interest and adverse events (AEs). Safety data are presented for patients receiving $120 \mathrm{mg}$ FlecIH during an 8-minute inhalation period. Conversion rate excludes patients who had flecainide present in their pre-dosing blood samples. Patients who did not convert to SR were offered alternative treatment per investigator's discretion.

Results : A total of 97 patients (34.0\% female) with a mean age of 60.4 years (range: 26,84$)$, mean weight of $87.5 \mathrm{~kg}$ (range: $57.5,150$ ) and mean height of $179.9 \mathrm{~cm}$ (range: 156,202$)$ were included. The conversion rate at 90 minutes post-dose was $47.5 \%(95 \%$ CI: 36.6, 58.4) and the median time to conversion was 14.6 (IQR: 20.7) minutes from start of inhalation. Mean \pm SD Cmax was similar in patients whose AF converted $(333 \pm 222 \mathrm{ng} / \mathrm{mL})$ and in patients whose AF failed to convert to SR $(370 \pm 229 \mathrm{ng} / \mathrm{mL})$; no patient had a Cmax [?] $1000 \mathrm{ng} / \mathrm{mL}$. Mean+-SD $\Delta$ QRSmax was similar in patients whose AF converted (8.0 $\pm 4.7 \mathrm{msec})$ and in patients whose AF failed to convert to SR $(7.9 \pm 6.6 \mathrm{msec})$. The most frequent AEs were similar in patients whose AF converted and in patients whose AF failed to convert to SR: cough (37.5\% vs. $33.3 \%)$; oropharyngeal discomfort (12.5\% vs. $5.3 \%)$; oropharyngeal pain ( $7.5 \%$ vs. $8.8 \%$ ); and salivary hypersecretion ( $2.5 \%$ vs. $8.8 \%$ ), respectively; none led to discontinuation of inhalation. Nearly all CVEs (see table) were transient and asymptomatic; none required treatment and all resolved without sequelae, including those considered serious.

Conclusions : Inhalation of $120 \mathrm{mg}$ FlecIH was safe, and the efficacy was similar to that reported for oral and IV administration. Patients whose AF failed to convert to SR with FlecIH did not experience any adverse consequences that required treatment or limited subsequent treatment of their AF.

AFS2022-32

Uploaded File(s)

CV Events (CVEs) of Special Interest for $120 \mathrm{mg}$ FlecIH by AF Conversion Status

CV Events (CVEs) of Special Interest for $120 \mathrm{mg}$ FlecIH by AF Conversion Status

Atrial Flutter 1:1 AV Conduction

Bradycardia

Hypotension

Sinus Pause Post-cardioversion

Ventricular Tachycardia

Note: Number of subjects with at least 1 CVEs in each category. Nearly all CVEs were transient and asymptomatic; none

\section{AFS2022-33Submission Number: 1171344}

Abstract Title: Characterizing lesion formation of temperature-titrated radiofrequency dosage with a novel diamond tip ablation system

Author(s): William R. Maddox, MD

Co-Author(s):

Tarvinder Dhanjal, MD, PhD, FRCP, FESC

Michael Getman, MS, FHRS - Principal Medical Science Liaison, Medronic, Inc.

H. Thomas McElderry, MD - University of Alabama Birmingham

Megan Schmidt, PhD - Medronic, Inc.

Atul Verma, MD - Southlake Regional Health Centre 
University of Alabama Birmingham

University of Alabama Birmingham 930B Faculty Office Tower, 510 20th Street South Birmingham, AL, USA

\section{Introduction | Objectives:}

The success of catheter ablation as a treatment for atrial fibrillation depends on transmural lesions. The DiamondTemp Ablation (DTA) system is a novel temperature-controlled irrigated radiofrequency (RF) ablation system that accurately measures tip-tissue temperature and modulates power in real-time. Lesion characteristics at different RF durations with this system have not previously been systematically defined within an animal model. We sought to evaluate lesion characteristics of the DTA system when varying the application duration with a "temperature-titrated" methodology.

Methods: The DTA system was used to deliver discrete lesions in all chambers of the heart using an in vivo canine model. Ablation parameters were set at $50 \mathrm{~W}$ with a temperature set point of $60^{\circ} \mathrm{C}$. Upon reaching $50^{\circ} \mathrm{C}$ atrial ablations were extended by 3 or $5 \sec (\mathrm{A} 3$ and $\mathrm{A} 5)$ and ventricular ablations by 10 or $15 \sec (\mathrm{V} 10$ and V15).

Results: In total, 59 atrial lesions and 28 ventricular lesions were analyzed in 4 canines. The ablation characteristics and resultant lesion dimensions for A3, A5, V10, and V15 are as follows: ablation duration $(5.1 \pm 1.5 \mathrm{sec}, 7.4 \pm 1.3 \mathrm{sec}, 12.8 \pm 2.8 \mathrm{sec}$, and $17.9 \pm 3.4 \mathrm{sec})$, impedance decrease $(13 \pm 5 \Omega, 15 \pm 5 \Omega, 17 \pm 4$ $\Omega$, and $18 \pm 6 \Omega$ ), average temperature $\left(55.2 \pm 3.2^{\circ} \mathrm{C}, 55.6 \pm 2.7{ }^{\circ} \mathrm{C}, 55.6 \pm 3.7^{\circ} \mathrm{C}\right.$, and $\left.57.5 \pm 3.2^{\circ} \mathrm{C}\right)$, depth $(2.2 \pm 0.8 \mathrm{~mm}, 2.0 \pm 1.1 \mathrm{~mm}, 5.8 \pm 1.6 \mathrm{~mm}$, and $6.0 \pm 1.6 \mathrm{~mm})$, and width $(5.8 \pm 1.7 \mathrm{~mm}, 6.4 \pm 1.7 \mathrm{~mm}, 7.5 \pm 1.6 \mathrm{~mm}$, and $8.0 \pm 1.5 \mathrm{~mm})$. No steam pops, char, or coagulum were observed.

Conclusions: Using the DTA system, 5 second applications were sufficient to create transmural atrial lesions. Ventricular lesion depths of approximately $6 \mathrm{~mm}$ were created with short RF applications of 13-18 seconds.

\section{AFS2022-33}

\section{Uploaded File(s)}

Atrial \& Ventricular Lesions from the DiamondTemp Ablation System in a Canine Model 

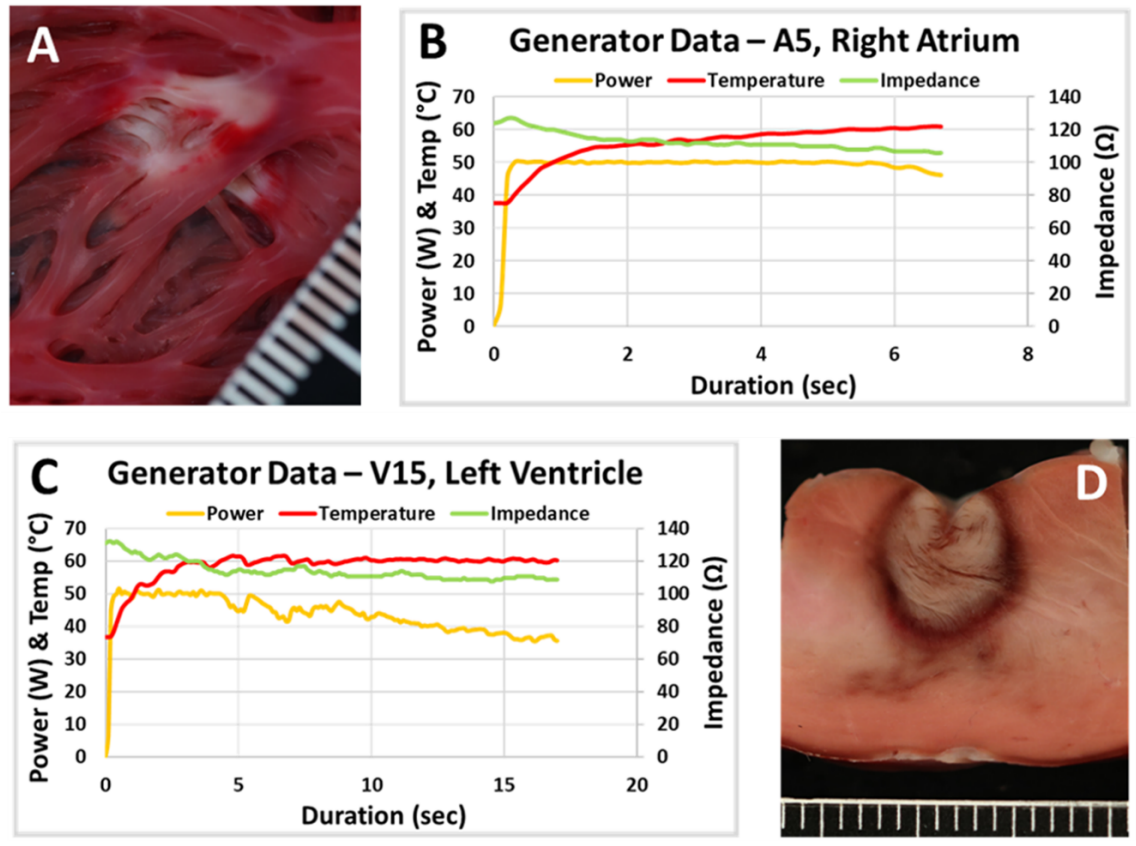

\section{AFS2022-34Submission Number: 1171373}

Abstract Title: Procedural Efficiencies Using a Novel Diamond Tip Radiofrequency Ablation Catheter When Treating Atrial Fibrillation

Author(s): Petr Neuzil, MD, PhD

Co-Author(s):

Herve Poty, MD

Christian de Chillou, MD, PhD

Jan Petru, MD

Michael Getman, MS, FHRS - Principal Medical Science Liaison, Medronic, Inc.

Shufeng Liu, MS - Sr. Prin. Statistician, Medronic, Inc.

Moritoshi Funasako, MD, PhD

Alexis Durand-Dubief, MD

Stephane Combes, MD

Jean-Paul Albenque, MD

Na Homolce Hospital

Na Homolce Hospital, Roentgenova 37/2 15000 Prague 5 Czech Republic

Introduction | Objectives: As radiofrequency (RF) ablation technologies evolve and practitioners look to improve treatment options for atrial fibrillation ( $\mathrm{AF}$ ), a learning curve is generally observed. The novel DiamondTemp Ablation (DTA) system, a diamond tip, irrigated, RF ablation system, reported procedural efficiencies and a short learning curve in the DIAMOND-AF trial. We sought to further characterize procedural efficiency in the FASTR-AF trial. 
Methods: FASTR-AF (NCT03626649) was a prospective, single-arm, multicenter trial to evaluate the new FASTR generator. The three sites in the FASTR-AF study also participated in DIAMOND-AF. The timedependent procedural characteristics from the DIAMOND-AF trial are compared to those from FASTR-AF.

\section{Results:}

In total, 34 paroxysmal AF subjects (male $70.6 \%$, age $62.9 \pm 10.5$ yrs, PAF history $4.2 \pm 4.7$ yrs, LA size 4.3 $\pm 0.8 \mathrm{~cm})$ underwent treatment with the DTA system in the FASTR-AF trial. The same centers enrolled 88 subjects (with similar demographics) in DIAMOND-AF. Total procedure time, LA dwell time, total RF time, and individual RF application durations were significantly shorter in the FASTR-AF cohort compared to the DIAMOND-AF cohort $(76.9 \pm 21.9$ vs $92.6 \pm 29.2 \mathrm{~min}, 56.9 \pm 17.8$ vs $72.0 \pm 25.7 \mathrm{~min}, 10.4 \pm 3.5$ vs $15.0 \pm 4.9 \mathrm{~min}$, and $10.3 \pm 3.3$ vs $13.6 \pm 2.9 \mathrm{sec}$, respectively, all p-values $<0.01)$. There was no difference in the number of RF applications or fluoroscopy time between study cohorts.

Conclusions: FASTR-AF time-dependent procedural characteristics were significantly shorter than those observed during the DIAMOND-AF trial demonstrating the procedural efficiency gains over time for experienced users.

AFS2022-34

Uploaded File(s)

Procedural Efficiencies Using the DiamondTemp Ablation System 

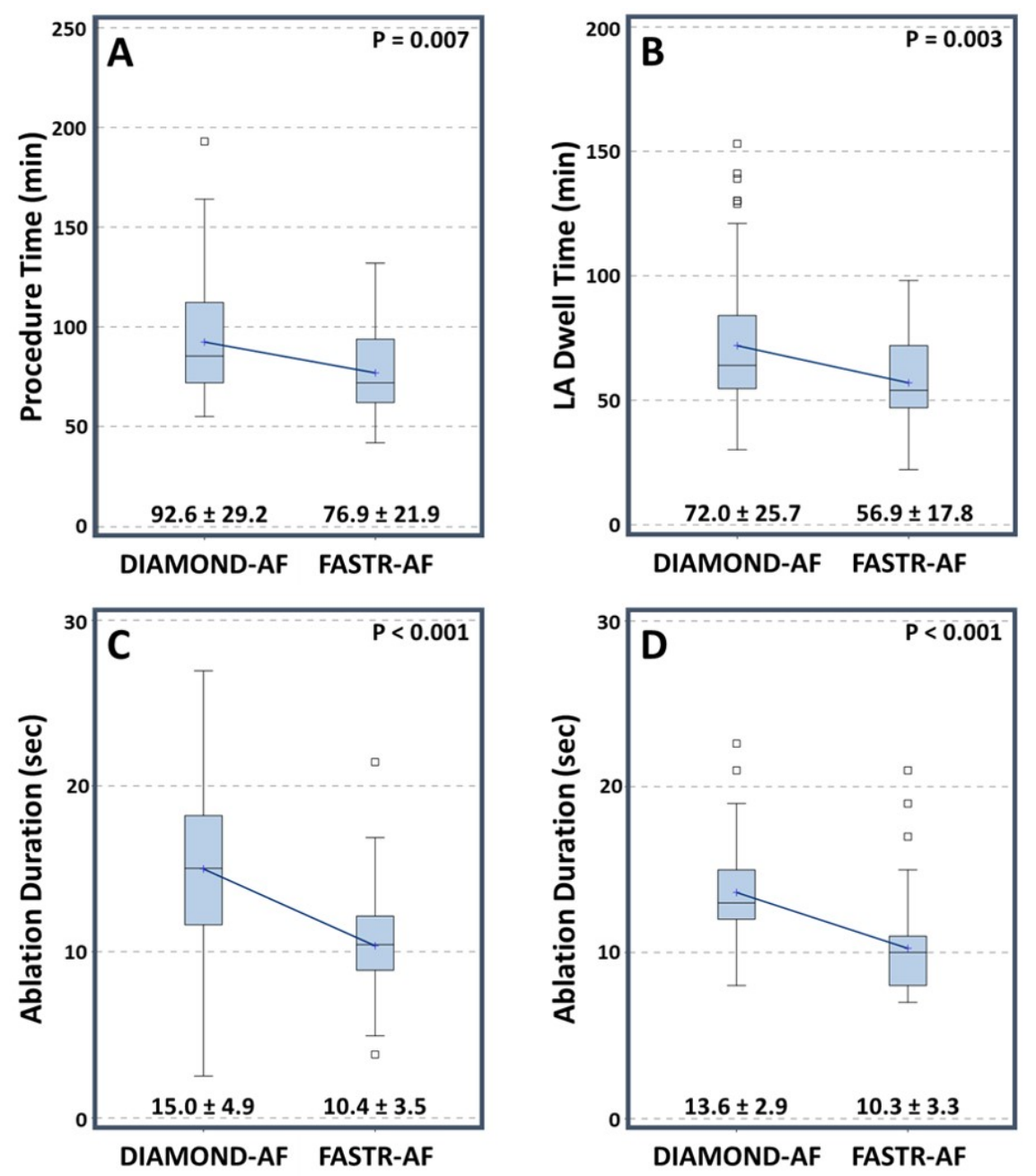

AFS2022-35Submission Number: 1171634

Abstract Title: High Power Short Duration Temperature Controlled Ablations Integrated with 3D Mapping \& Navigation System

Author(s): Paul Kessman, MSEE

Co-Author(s):

Watson Finney, BS

Amanda Mallon, BS

Moritoshi Funasako, MD, PhD

Petr Neuzil, MD, PhD

Jan Petru, MD

Usman Siddiqui, MD

Vivek Reddy, MD

CardioNXT 
12011 N. Tejon St., Westminster, CO

Introduction | Objectives:

High Power Short Duration RF ablation (HPSD) is gaining traction in cardiac arrythmia ablations. HPSD facilitates delivery of energy to tissue before edema sets in, impeding efficacious therapy delivery. New temperature controlled ablation systems are able to better measure tissue temperature during ablation and efficiently modulate power. One such system is the DiamondTemp ablation system (Medtronic, Minneapolis, MN, USA), which uses industrial grade diamonds to shunt energy during RF delivery allowing thermocouples to measure actual tissue temperature during ablation.

It would be useful to navigate the DiamondTemp catheter and observe data from the ablation generator on a 3D Mapping \& Navigation System during ablation.

Objectives are to display the location of the DiamondTemp catheter on a 3D Mapping and Navigation System, the iMap System (CardioNXT, Boulder, CO, USA). Display Temperature, Power, Impedance drop, and Duration from the DiamondTemp ablation generator on the iMap System. Visualize temperatures achieved in the tissue to assess areas of potential gaps.

\section{Methods:}

These cases were performed as part of a pilot study (DYNALINK) to evaluate the iMap System for safety in an effort to obtain CE Mark. Feasibility to navigate the DiamondTemp catheter and display tissue temperature data on the iMap System was evaluated as a secondary objective.

\section{Results:}

The iMap System navigated the DiamondTemp catheter in 25 patients. The iMap System was able to track an accurate representation of the DiamondTemp catheter, including the cap electrode, which is capable of minimizing far field signal detection (see Figure 1A and 1B). The iMap System was able to display ablation data in real-time from the DiamondTemp generator (see Figure 1C and 1D). Tissue temperature data was displayed in the form of a colored map with three colors representing temperature thresholds achieved in the tissue. Physicians were able to use the map as a tool to locate areas of optimal therapy delivery and areas of potential gaps (see Figure 1E).

\section{Conclusions:}

Visualizing DiamondTemp catheter position and ablation parameters on the iMap System facilitates efficient delivery of temperature controlled ablation and mitigates distraction of physician eyesight from the patient.

\section{AFS2022-35}

Uploaded File(s) 


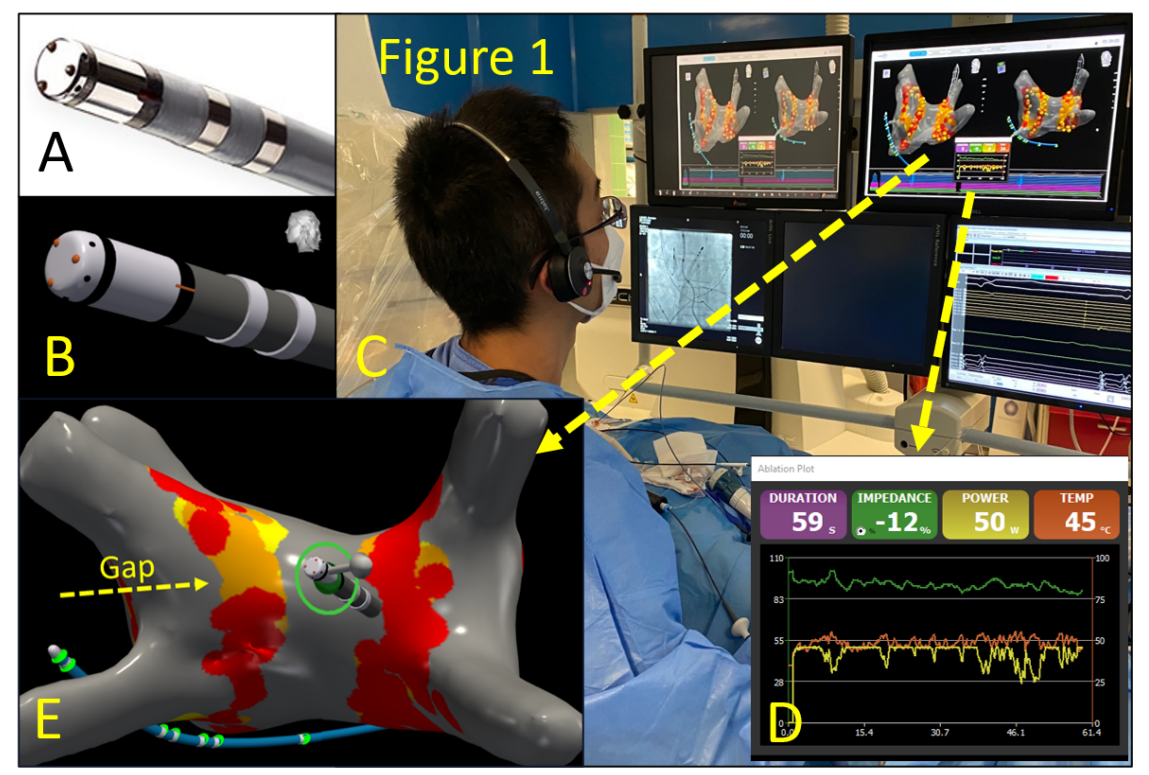

\section{AFS2022-36 Submission Number: 1173116}

Abstract Title: Pulsed Field Ablation Integrated with 3D Mapping \& Navigation System

Author(s): Paul Kessman, MSEE

Co-Author(s):

Jerome Edwards, MBA

Moritoshi Funasako, MD, PhD

Petr Neuzil, MD, PhD

Jan Petru, MD

Vivek Reddy, MD

\section{CardioNXT}

12011 N. Tejon St., Westminster, CO

\section{Introduction | Objectives:}

Pulsed Field Ablation (PFA) is an exciting new modality for ablating cardiac arrhythmias. The benefits of PFA include the lack of collateral damage to critical structures such as the esophagus. There are several PFA systems currently being developed. One in particular has obtained CE Mark, the Farapulse System (Boston Scientific, Boston, MA, USA). PFA delivery with this system today is predominantly being navigated with fluoroscopy (X-Ray) and intracardiac echo (ICE).

Dosing protocols of PFA systems involve positioning the catheter within the pulmonary veins (PVs) in different configurations (basket, flower, etc.) and delivering pulse trains, clocking the catheter and delivering additional pulses.

It would be useful to visualize the PFA catheter utilizing a 3D Mapping \& Navigation system during procedures to understand the orientation of the catheter within anatomy to properly administer dosing protocols associated with PFA, potentially reducing ionizing radiation. 
Objectives are to utilize a new hybrid electromagnetic and impedance based 3D Mapping \& Navigation system (CardioNXT iMap System, Boulder, CO, USA) to navigate the Farapulse catheter during delivery and dosing of PFA.

\section{Methods:}

These cases were performed as part of a pilot study (DYNALINK) to evaluate the iMap System for safety in an effort to obtain CE Mark. Feasibility of PFA navigation was evaluated as a secondary objective in the study.

\section{Results:}

The iMap System navigated the Farapulse catheter in 10 patients. The iMap System was able to accurately track and visualize the catheter in various configurations (see 1A and 1B). The iMap System was able to navigate the catheter to create a Left Atrial geometry suitable to visualize catheter orientation within the PVs and LAA. No additional mapping catheter was required. The iMap System was able to navigate the catheter during PFA delivery without lag or latency (see Figure 1C and 1D). Verification of isolation of the PVs and PW while preserving the LAA was confirmed with a post voltage map (see Figure 1E and $1 \mathrm{~F}$ ).

\section{Conclusions:}

The future of PFA delivery will likely be a combination with 3D Mapping \& Navigation in order to facilitate dosing protocols and reduce the use of ionizing radiation. The iMap System is one such system that can be used for this purpose of PFA delivery.

AFS2022-36

\section{Uploaded File(s)}

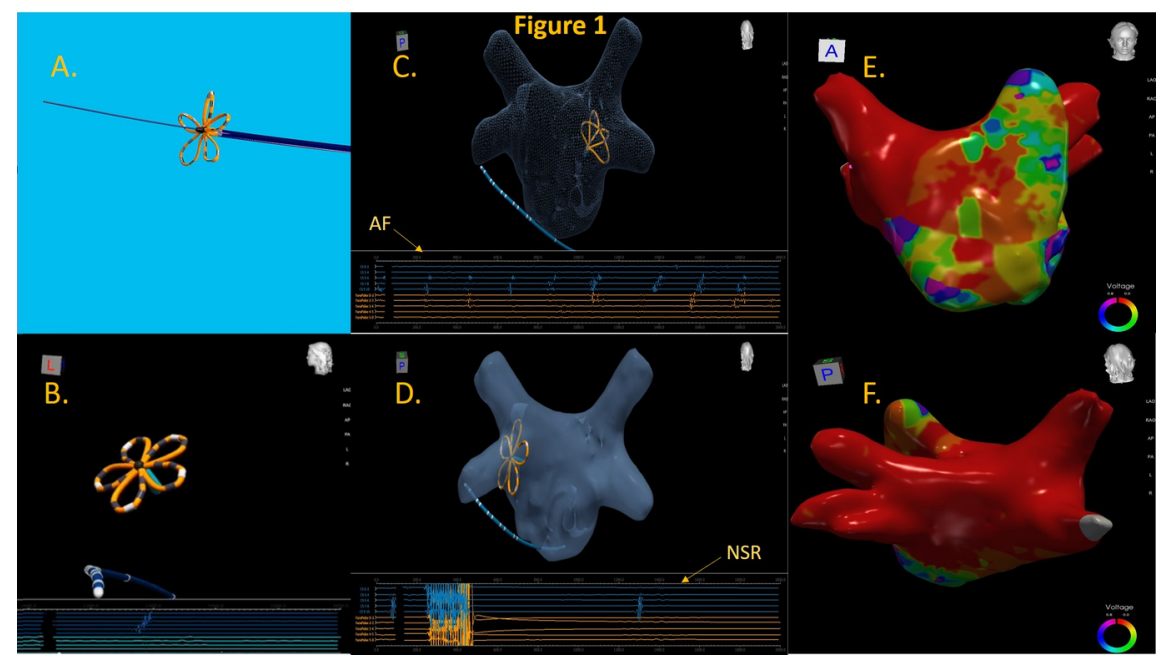

AFS2022-37Submission Number: 1173153

Abstract Title: Preclinical Safety Evaluation of a 'Map and Ablate' Multielectrode Array using Pulsed Field Ablation.

Author(s): Iwanari Kawamura, MD

Co-Author(s):

Vivek Reddy, MD 
Daniel Reinders, MSc

Srinivas Dukkipati, MD

Petr Neuzil, MD

Jacob Koruth, MD

Icahn School of Medicine at Mount Sinai

One. Gustave. L. Levy Place, Box 1030, New York, NY 10029

\section{Introduction | Objectives:}

A multielectrode spherical array (Globe; Kardium inc) capable of one-shot 'mapping and ablation' has previously been shown able isolate PVs using radiofrequency (RF) energy. It has now been designed able to also deliver pulsed field (PF) energy. We performed a detailed safety evaluation of PFA in heathy swine.

\section{Methods:}

Three healthy swine underwent transseptal access under general anesthesia. PF ablation $(1.6-2 \mathrm{kV})$ was delivered using the array in both gated and ungated PFA modes to create lesions around the right superior pulmonary vein (RSPV), and determine ablation transmurality. Additionally, a modified 'single-rib' smaller form factor version of this catheter representative of the array was used to deliver 8 PF lesions deep within the superior vena cava, to evaluate venous stenosis, and from the inferior vena cava onto the forcefully deflected esophagus (6 applications/esophagus) to assess esophageal safety. A single additional swine underwent temperature-controlled RF ablation (single application, $63^{\circ} \mathrm{C}$ for 3 minutes) from the inferior vena cava using the 'single-rib' to serve as a control (non-survival study). The animals that received PF were survived for 30 days and were evaluated for venous stenosis, phrenic palsy with pathological exam of the heart and esophagus.

\section{Results:}

All three swine completed the survival period without any additional in-life observations. A total of 36 sections were analyzed and 33 of $36(91.7 \%)$ lesions were noted to be transmural. During PFA, phrenic activation and muscular stimulation was mild. There were no ventricular arrhythmias with either gated or ungated mode. In follow up, there was no stenosis seen on contrast venography or instances of phrenic palsy. The esophagus was serially cross-sectioned (44 sections in total) and gross evaluation of serosal/mucosal surfaces was normal in the PFA group (histology pending), whereas a long linear thermal lesion was seen with RF (Figure ).

\section{Conclusions:}

This novel spherical array catheter can rapidly map and ablate atrial tissue using pulsed electrical fields without incurring venous stenosis or phrenic palsy, despite ablation deep within the superior vena cava and without causing esophageal injury despite ablation directly atop the esophagus.

\section{AFS2022-37}

\section{Uploaded File(s)}




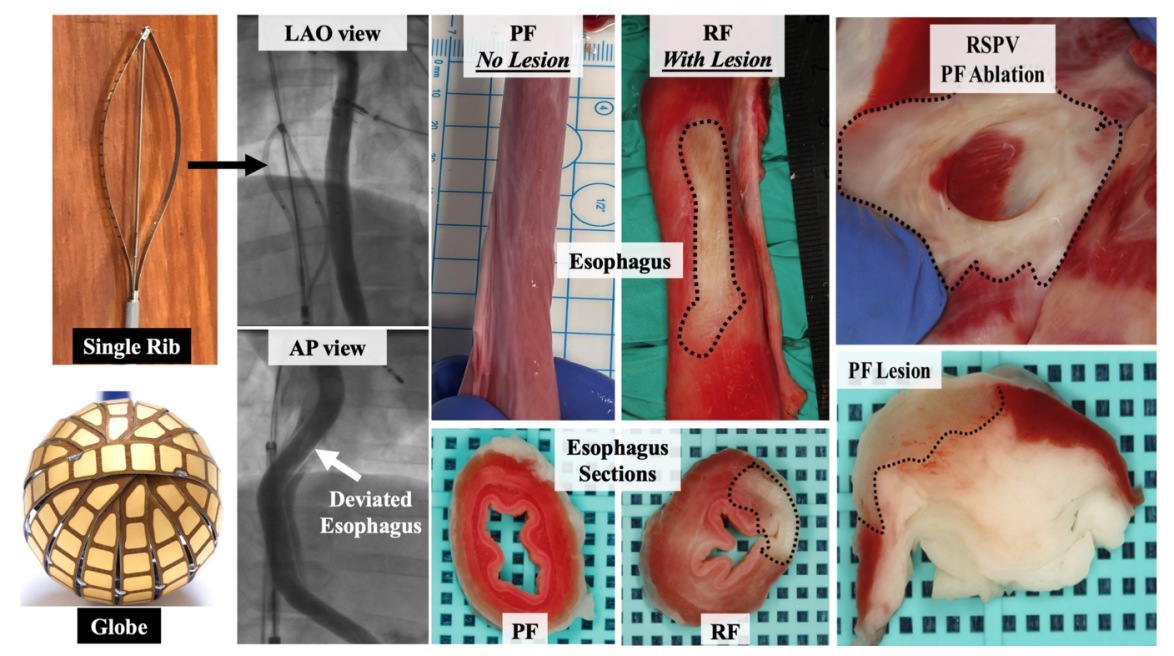

AFS2022-38Submission Number: 1173161

Abstract Title: 'Worst-Case' Esophageal Injury Assessment: Pulsed Field vs Radiofrequency Ablation using a Focal, Contact Sensing, Gold-Tipped Catheter.

Author(s): Iwanari Kawamura, MD

Co-Author(s):

Vivek Reddy, MD

Eric Sauter, BS

Steven Mickelsen, MD

Petr Neuzil, MD

Srinivas Dukkipati, MD

Jacob Koruth, MD

Icahn School of Medicine at Mount Sinai

One. Gustave. L. Levy Place, Box 1030, New York, NY 10029

\section{Introduction | Objectives:}

Esophageal injury with pulsed field ablation (PFA) using several catheter designs has been reported to be minimal compared to thermal energy. But it is possible (indeed likely) that this finding is specific to PFA catheter designs and PF waveforms, and therefore individual catheter-specific assessments of esophageal safety are needed. We compared the effect of PF with radiofrequency (RF) delivered atop theporcine esophagus using an $8 \mathrm{Fr}, 3.5 \mathrm{~mm}$ force-sensing gold tip catheter.

\section{Methods:}

Under general anesthesia, the esophagus in 7 swine, was deflected towards the inferior vena cava using a esophageal deviation device (DV8; Manual Surgical Sciences Inc) under fluoroscopic guidance. An 8.5Fr deflectable sheath was used to deliver the study catheter (AcQBlate Force; Acutus Medical Inc) via femoral venous access. Bipolar/biphasic PF atrial applications $(n=36)$ were delivered to confirm ablative efficacy. Six of 7 swine received 10.8 $\pm 1.2 \mathrm{PFA} /$ per swine esophagus, whereas the one remaining swine received $9 \mathrm{RF}$ applications using the same catheter (30W, up to 60 seconds). All PF animals were survived to 4 weeks 
whereas the RF swine was survived for only 2 weeks to minimize potential for distress related to esophageal injury. After sacrifice, the heart and esophagus were explanted and submitted for pathology.

\section{Results:}

The mean increase in weight was $7.4 \pm 3.3 \%$ vs only $0.8 \%$ in the PF and RF cohorts, respectively. Upon necropsy, grossly-identifiable lesions were seen for 29 of $36 \mathrm{PF}$ applications. The mean length, width and depth of lesions were $12.7 \pm 3.5 \mathrm{~mm}, 7.5 \pm 2.3 \mathrm{~mm}$ and $3.5 \pm 1.2 \mathrm{~mm}$, respectively. On the esophagus, PF cohort demonstrated no evidence of esophageal lesions (serosa and lumen); in contrast, the RF cohort had clear esophageal injury (Figure ). A penetrating ulcer measuring $12.6 \times 7.1 \mathrm{~mm}$ with extension into the adjacent lung was identified in an RF-treated animal, confirming the validity of the injury-assessment model.

\section{Conclusions:}

This report demonstrates that at ablative doses, PFA delivered using a focal force-sensing gold-tip catheter resulted in no grossly-visible esophageal damage, in contrast to obvious thermal damage with RFA. Histopathological confirmation of these findings is awaited.

AFS2022-38

\section{Uploaded File(s)}

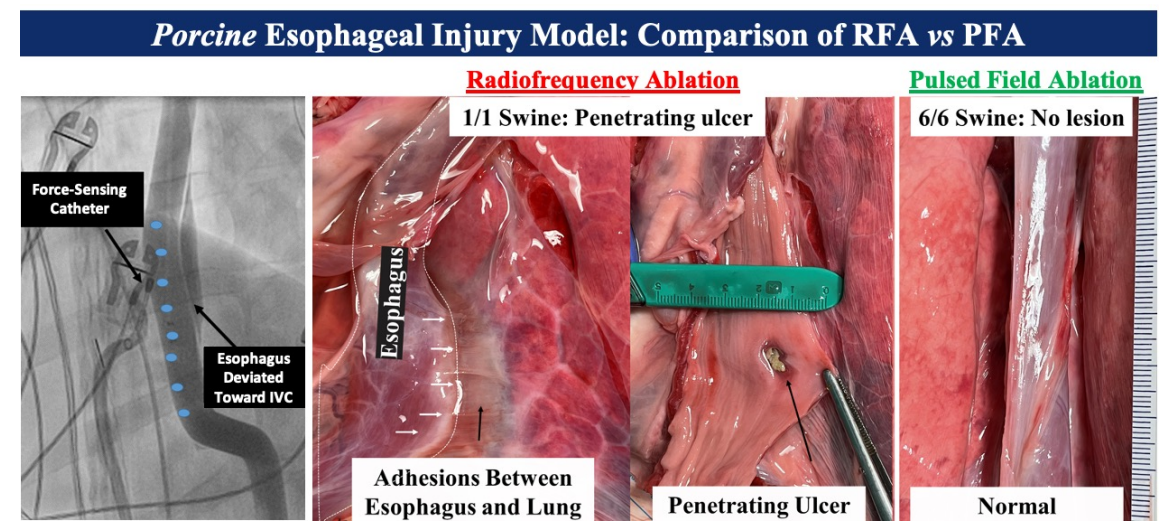

AFS2022-39Submission Number: 1174488

Abstract Title: Contractile Response of Esophagus to Pulsed Field Ablation: A Novel Class Effect

Author(s): Jacob Koruth, MD

Co-Author(s):

Iwanari Kawamura, MD

Jan Petru, MD

petr Neuzil, MD

Vivek Reddy, MD

Icahn School of Medicine at Mount Sinai

One Gustave L. Levy Place, box 1030, New York, NY, 10029, USA

\section{Introduction | Objectives:}

Pulsed field ablation (PFA) from within the left atrium has been shown to spare esophageal tissue via its unique non-thermal ablative mechanism coupled with its relative specificity for myocardial tissue. We 
present preclinical and clinical evidence of a novel mechanism which may contribute to esophageal sparing - a contractile/retractile response of the esophagus to PFA pulses, common to four PFA technologies.

\section{Methods:}

Under general anesthesia, the esophageal lumen was intubated with an orogastric tube and the lumen defined by iodinated contrast for all procedures. In 3 of 4 preclinical studies, an esophageal deviation balloon (DV8, Manual Surgical Sciences Inc) was used to appose the esophagus against the inferior vena cava - where the PFA catheter was situated for ablation. In patients, PFA was delivered atop the esophagus during its relaxed state (wide lumen full of contrast). The esophageal response was recorded on fluoroscopy. Four PFA systems were tested: a pentaspline catheter (Farapulse Inc), an 8 Fr gold tip force-sensing focal catheter (Acutus Medical Inc), a focal $9 \mathrm{~mm}$ lattice tip catheter (Affera Inc), and a $30 \mathrm{~mm}$ multielectrode spherical array (Kardium Inc).

\section{Results:}

All applications resulted in esophageal contraction, and this response was demonstrated repeatedly. In preclinical studies, we observed rapid rhythmic contractions of the esophagus in regions in proximity to the PFA catheter, whereas all clinical cases demonstrated a more diffuse contraction that extended along the length of the esophagus. The figure demonstrates three still-frames with progressive esophageal contraction in a patient immediately after PFA.

\section{Conclusions:}

Esophageal contraction/retraction appears to occur consistently with various PFA technologies, likely a result of direct stimulation of the esophageal musculature. By potentially reducing the proximity of the esophagus to the ablative electric field, this may represent yet another mechanism by which esophageal damage is minimized. This may also have relevance for esophageal instrumentation during PFA cases, including the potential for esophageal reflux during the procedure.

\section{AFS2022-39}

\section{Uploaded File(s)}

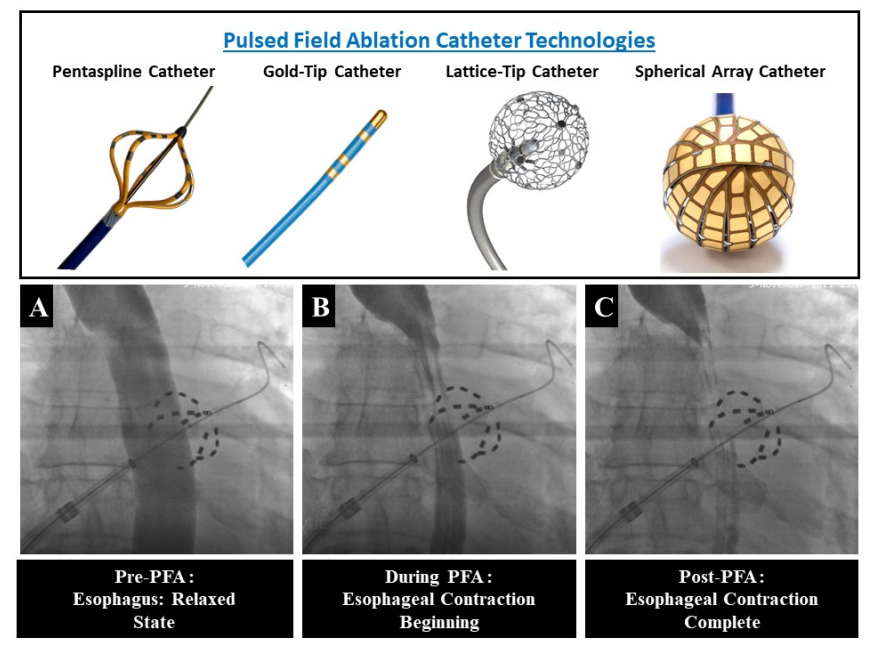

AFS2022-40 Submission Number: 1173174

Abstract Title: Histological Assessment of Pulmonary Vein Isolation using an Expandable, Lattice Shaped "Single-Shot" Pulse Field Ablation Catheter 
Author(s): Jacob Koruth, MD

Co-Author(s):

Iwanari Kawamura, MD

Srinivas Dukkipati, MD

Vivek Reddy, MD

Icahn School of Medicine at Mount Sinai

One Gustave L. Levy Place, box 1030, New York, NY, 10029, USA

Introduction | Objectives:

Single shot approaches to pulmonary vein isolation are attractive as they offer improvements in procedural efficiency. We have previously demonstrated preclinical feasibility of thoracic vein (PV, SVC) isolation using a novel, expandable lattice shaped one-shot pulsed field ablation (PFA) catheter in swine. We aimed to assess the histological findings of ablated thoracic veins (TV) using this catheter.

\section{Methods:}

The study catheter (SpherePVI; Affera Inc) was used to isolate TVs under general anesthesia in swine. Two cohorts of swine were treated and survived for 1 and 5 weeks, respectively. In cohort 1, an initial PFA dose ("PULSE2") was used in 6 swine for isolation of both the superior vena cava (SVC) and the right superior pulmonary vein (RSPV) and in 2 swine for SVC isolation only. In cohort 2, a final PFA dose ("PULSE3") was used in 5 swine to isolate the SVC, RSPV and LSPV. A total 8 veins (RSPV [n=4], and SVC [n=4]) were chosen from 4 swine in cohort 2 for detailed histological assessment. Ablated thoracic veins were identified, cut open and trimmed to get evenly spaced circumferential sections along the long axis of the vein (4-8 sections/PV).

\section{Results:}

In cohort 1, durable isolation was observed in 6 of 6 RSPVs and 6 of 8 SVCs. A total of 32 and 52 sections from the SVC and RSPVs, respectively, were analyzed. Lesions were readily identified and transmurality was noted in all 84 of $84(100 \%)$ sections, with a mean depth of $4.0 \pm 2.0 \mathrm{~mm}$ with thickness of up to $10 \mathrm{~mm}$ (Figure A \& B ). Viable blood vessels and nerves within the zone of ablation treatment were identified (Figure C \& D ). In cohort 2, 14 of 15 veins ( 5 of 5 SVC, 5 of 5 RSPV, and 4 of 5 LSPV) demonstrated durable isolation. Histological examination of all sections of RSPV $(n=31)$ and SVC ( $n=34)$ from cohort 2 revealed circumferential transmural ablation (75 of 75, 100\%). There was minimal inflammation, no evidence of mineralization and viable blood vessels and nerves within and around ablated areas were seen.

\section{Conclusions:}

This novel PFA catheter, using its proprietary waveform, provides excellent transmurality rates with evidence of sparing of blood vessels and nerves characteristic of pulsed field ablation.

\section{AFS2022-40}

Uploaded File(s) 


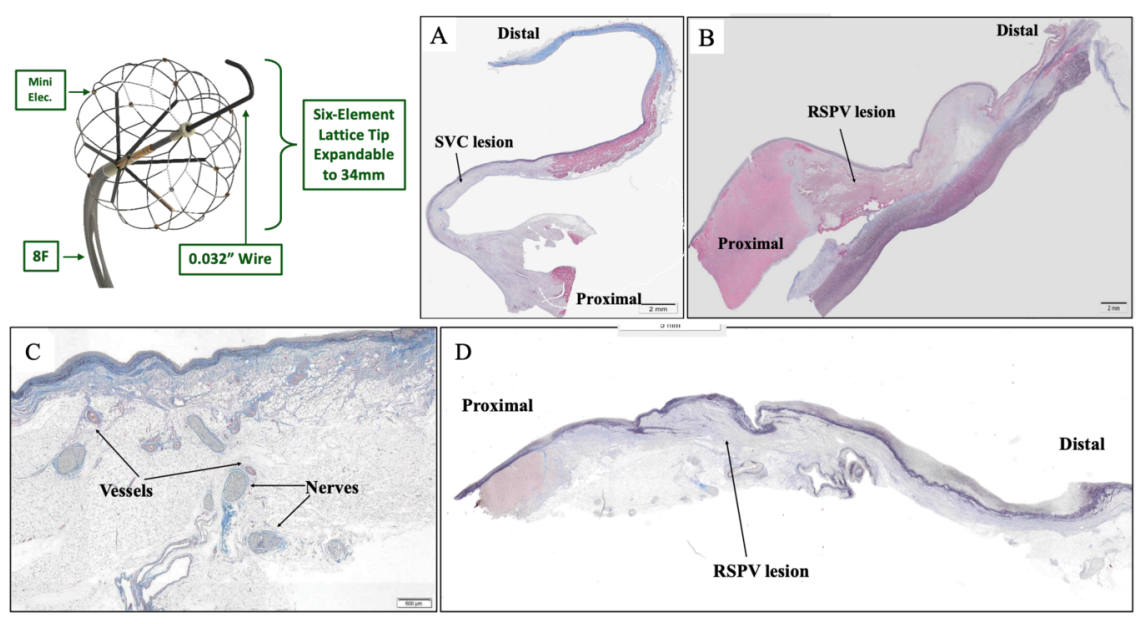

AFS2022-41Submission Number: 1173358

Abstract Title: A novel mapping tool identifies fast and consistent discrete targets for persistent atrial fibrillation

Author(s): Decebal G. Latcu, MD

Co-Author(s):

Sofia Milanese, Master Degree - Boston Scientific

Amanda Kingston, MS - Boston Scientific

Rudy Gelb-Bicknell, BS - Boston Scientific

Sarah Gutbrod, PhD - Boston Scientific

Bogdan Enache, MD

Nazih Benhenda, MD

Centre Hospitalier Princesse Grace

1,Avenue Pasteur, Monaco 98000

Introduction | Objectives:

Developing ablation strategies in persistent atrial fibrillation (persAF) is still a challenge. To facilitate ablation target identification, a new embedded high-definition mapping tool calculates and displays (scatterplot) local cycle length (LCL) and local activation time within that LCL (Duty Cycle) for consistent electrograms (EGM).

Methods: Case Study

Results: A 75-year-old male was diagnosed with paroxysmal AF in 2010 and underwent pulmonary vein isolation (PVI) catheter ablation in 2017 after failure of the medical treatment. Symptomatic AF recurred in a persistent form in June 2021 and electrical cardioversion restored sinus rhythm (SR) for only $48 \mathrm{~h}$. The patient presented in AF at the time of a new catheter ablation in December 2021. Left atrial mapping (RHYTHMIA HDx, Orion) showed a relatively slow and organized LCL, with a majority of the LCLs varying between 200 and 250ms, with the LPVs durably isolated, but short-segment reconnection of RPVs. The integrated novel toolset (Ockham) highlighted a single driver in the RSPV identified by strict criterion on the scatterplot (green zone). The EGMS were characterized by consistent fast activation (LCL: 151-182ms, the 
fastest site in the LA) and high Duty Cycle ([?]90\%). This region corresponded to the area of a microreentry on the activation map. Ablation of the driver restored SR. Remap of the PVs was performed under pacing to locate the gap and guide additional ablations to ensure re-isolation. The procedure had no complications.

Conclusions: PersAF patients are known to have high variability in arrhythmogenic sites. This case is a pure model of a unique driver perpetuating AF. It highlights the effectiveness of a novel mapping tool for identification of ablation targets based on EGM characteristics for an individualized approach to persAF ablation.

\section{AFS2022-41 Uploaded File(s)}

Figure 1

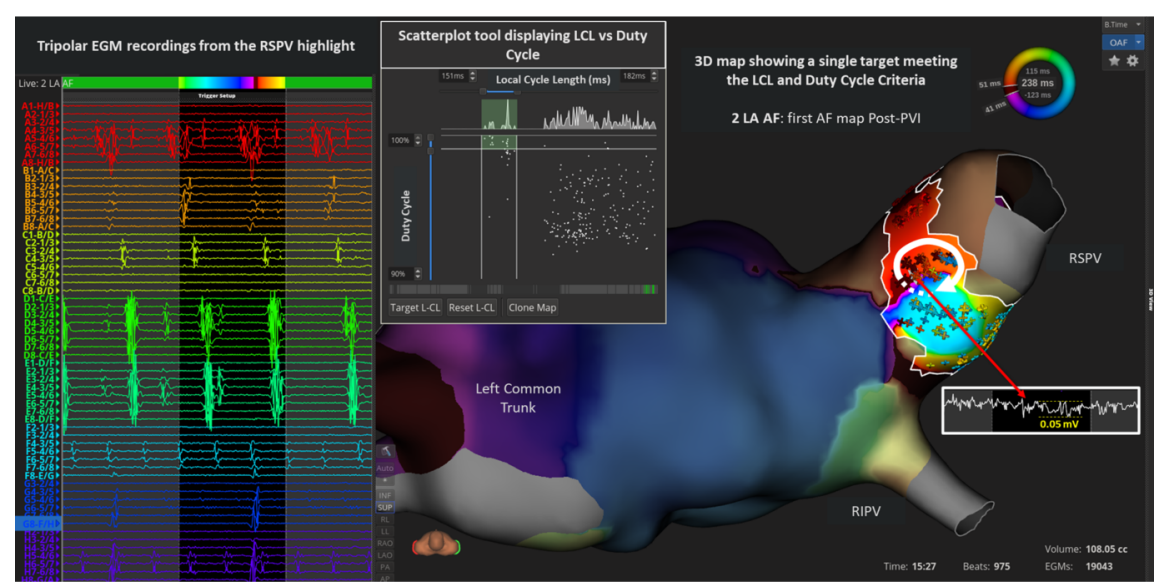

AFS2022-42Submission Number: 1173472

Abstract Title: Flecainide Induced Interstitial Lung Disease: A Case Report and Review of the Literature Author(s): Spencer Sherman, BA

Co-Author(s):

Andrew Sherman, MD - Chief, BayCare Health Systems

Franccesca Pickmans, BS - Research Associcate, University of South Florida

Natarajan Subramanian, MD - Tampa Pulmonary \& Sleep Specialists

Kevin Makati, MD - Director, BayCare Health Systems

Tampa Cardiac Specialists

4211 Van Dyke Road Suite 205 Lutz, FL 33558

\section{Introduction | Objectives:}

Flecainide has been used frequently as an antiarrhythmic (AA) for atrial fibrillation (AF) since its synthesis in 1972 however rarely implicated and reported as a cause of pulmonary toxicity and the development of interstitial lung disease (ILD). Case reports in the literature have suggested an acute onset however rarely described after chronic usage. The course of disease and the ability to recover from this uncommon complication has not been clearly delineated. Here we report a case of delayed drug-induced ILD following Flecainide treatment and summarize the relevant literature.

Methods: N/A 


\section{Results:}

The patient is a 65-year-old male with a prior history of persistent AF, dyslipidemia, and diabetes, who underwent a successful hybrid AF ablation in 2016. A pre-ablation CT scan performed at that time documented the absence of ILD. In 2020, he developed mitral annular flutter that was interrupted using radiofrequency to create an anterior lateral line. Flecainide was initiated prior to ablation and continued by patient's preference despite technical success. Nine months following the procedure, the patient had a coronary CT scan which retrospectively showed mild ground glass opacities. One year following Flecainide initiation, progressive dyspnea ensued and was investigated by pulmonary function testing showing restrictive changes compatible with ILD. A repeat CT scan was performed 18-mo after initiation of antiarrhythmic to investigate treatment refractory symptoms confirming fulminant ILD. As he did not take any other medications, Flecainide was discontinued, an empiric course of steroids was begun, and the patient had full resolution of his symptoms.

\section{Conclusions:}

Our report describes a rare complication of a commonly prescribed class IC AA with recommendations on detection, review of differential diagnoses, and treatment options. Prompt diagnosis is critical to management as irreversible lung fibrosis has been documented.

\section{AFS2022-42 Uploaded File(s)}

Timeline by CT scans Following Flecainide Exposure

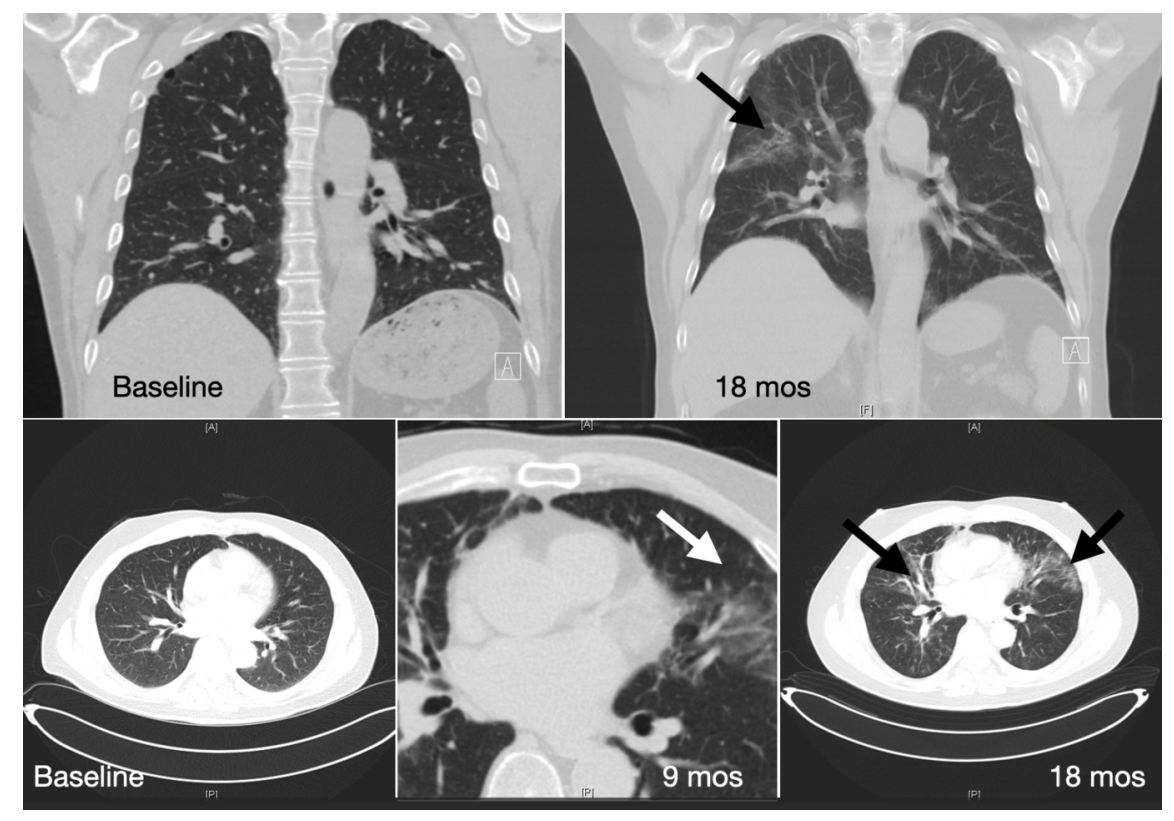

AFS2022-43Submission Number: 1173621

Abstract Title: The impact of active esophageal cooling on pulmonary vein isolation procedure duration in a single-hospital setting

Author(s): Christopher Joseph, BA

Co-Author(s):

Christopher Henry, MD

Ray Kawasaki, MD 
University of Texas Southwestern Medical School

5323 Harry Hines Blvd Dallas, TX 75390

Introduction | Objectives: A mainstay of treatment for patients with atrial fibrillation (AF) is pulmonary vein isolation, which involves risk of esophageal thermal injury. The traditional method of esophageal protection, luminal esophageal temperature (LET) monitoring involves temperature probes in the esophagus that set off alarms when the lumen reaches unsafe levels. Another method to reduce esophageal injury involves active cooling of the esophagus to prevent temperature spikes rather than monitor them. While there have been recent studies demonstrating limitations and possible harm when utilizing LET monitoring for esophageal protection, its impact on procedure duration has not been well characterized when compared to active cooling.

Methods: In this IRB-approved study, we collected data from patients undergoing pulmonary vein isolation at a single center beginning in February 2018 through March 2021. For each patient, we collected age, gender, method of esophageal protection used, and procedure duration. Then, we compared the procedure duration for operations before and after the implementation of active esophageal cooling as a replacement for LET monitoring with multi-sensor probes.

\section{Results:}

Data was collected from a total of 163 patients. LET monitoring was utilized in 108 patients, while active cooling was used in 55 patients. In the LET monitored group, mean duration was 179 minutes, and the median was 180 minutes. In the actively cooled group, mean procedure duration was 154 minutes, while the median duration was 145 minutes. This represents a mean reduction of 25 minutes or a $14 \%$ reduction in procedure duration $(\mathrm{p}<.001)$ and a median reduction of 35 minutes or a $19 \%$ reduction in procedure duration $(\mathrm{p}<.001)$.

Conclusions: In this single center study, the use of active esophageal cooling was associated with a significant reduction in overall procedure duration.

AFS2022-44Submission Number: 1162900

Abstract Title: Active esophageal cooling significantly reduces procedure time during ablation of both paroxysmal and persistent atrial fibrillation

Author(s): Christopher Joseph, BA

\section{Co-Author(s):}

Jacob Sherman, N/A

Westby Fisher, MD

Jose Nazari, MD

Alex Ro, MD

Mark Metzl, MD

University of Texas Southwestern Medical School

5323 Harry Hines Blvd Dallas, TX 75390

Introduction | Objectives: Pulmonary vein isolation (PVI) procedure time can be dependent on the method of esophageal protection used. Active esophageal cooling has shown reductions in procedure duration due to the avoidance of temperature alarms encountered with traditional luminal esophageal temperature (LET) monitoring. The impact of atrial fibrillation (AF) type, if any, is not as well characterized in existing literature. In this study we aimed to characterize the change in procedure duration when utilizing active 
cooling, and compare differences between patients with paroxysmal, persistent, and long-standing persistent atrial fibrillation.

Methods: This retrospective review evaluated patients with AF undergoing PVI from January 2018 to February 2020. For each patient, we recorded age, gender, AF type, and total procedure time, and then compared procedure lengths before and after the implementation of active esophageal cooling in lieu of LET monitoring.

Results: A total of 373 patients were included in this study. There were 161 patients with paroxysmal $\mathrm{AF}, 135$ with persistent $\mathrm{AF}$, and 77 with long-standing persistent $\mathrm{AF}$, atypical flutter, or atrial tachycardia. Mean procedure time in the paroxysmal group was 135 minutes in the LET monitored patients, and 105 minutes in the actively cooled patients, with an overall reduction of 30 minutes $(\mathrm{P}<.001)$. In the persistent AF group, mean duration was 154 minutes in the LET monitored group, and 122 minutes in the actively cooled group, with an overall reduction of 32 minutes $(\mathrm{P}<.001)$. Finally, in the long-standing persistent AF group, mean duration was 157 minutes in the LET monitored group, and 107 minutes in the actively cooled group, with an overall reduction of 50 minutes $(\mathrm{P}<.001)$.

Conclusions: The use of active esophageal cooling in PVI is associated with a significant reduction in procedure duration regardless of AF type.

\section{AFS2022-45Submission Number: 1174443}

Abstract Title: Active esophageal cooling is associated with improved freedom from arrhythmias at oneyear: a large hospital registry review

Author(s): Christopher Joseph, BA

Co-Author(s):

Danya Willms, BS

Gregory Francisco, MD

Annie Ruppert, RN

Charles Athill, MD

University of Texas Southwestern Medical School

5323 Harry Hines Blvd Dallas, TX 75390

\section{Introduction | Objectives:}

As the rates of pulmonary vein isolation (PVI) for the treatment of atrial fibrillation (AF) continue to rise, there has been an increase in innovation to reduce the burden on esophageal injury during posterior wall ablation. While there have been studies comparing esophageal injury severity in luminal esophageal temperature (LET) monitoring and active esophageal cooling, there is limited data on their impacts, if any, on ablation efficacy and freedom from AF. An increased continuity index, a function measuring the interruptions in posterior wall ablations, serves as a possible mechanism for a reduced freedom from AF when utilizing LET as a direct result of pauses from temperature alarms during the procedure. We sought to analyze followup AF recurrence data from patients who underwent PVI using LET monitoring and compare rates to those who underwent active esophageal cooling.

Methods: We accessed data from an existing large hospital-maintained registry for ablations that occurred over the last ten years. We then separated the registry data into groups based on whether the patients received LET monitoring or active esophageal cooling, and created Kaplan-Meier (KM) estimates of freedom from arrhythmias.

Results: A total of 544 patients received PVI for AF, AT or atrial flutter and had recorded follow up data. 319 patients received LET monitoring during their ablations, while 225 patients underwent active esophageal 
cooling during their ablations. KM estimates for freedom from arrhythmia were $41.5 \%$ for LET monitored patients, and $64.3 \%$ for patients receiving active esophageal cooling $(\mathrm{P}<.001)$.

Conclusions: In a 10-year large hospital registry review, the use of active esophageal cooling during PVI was associated with greater freedom from arrhythmia as compared to patients that underwent traditional LET monitoring.

\section{AFS2022-46Submission Number: 1173692}

Abstract Title: The effect of active esophageal cooling during left atrial ablation on postoperative chest pain - a updated survey of operators

Author(s): Christopher Joseph, BA

\section{Co-Author(s):}

James Daniels, MD

Ray Kawasaki, MD

Jose Nazari, MD

Kamala Tamirisa, MD

Mark Metzl, MD

University of Texas Southwestern Medical School

5323 Harry Hines Blvd Dallas, TX 75390

Introduction | Objectives: The use of active esophageal cooling during left atrial ablation has been associated with a reduction in incidence and severity of esophageal lesions when compared to luminal esophageal temperature (LET) monitoring. While this relationship has been investigated objectively through endoscopy, trends of decreased gastroparesis have also been reported. Given these findings, and the known pleiotropic effects of cooling in general, it is possible that the use of active cooling may also improve patient-reported post-ablation pain. In order to investigate this relationship, we surveyed electrophysiologists utilizing active cooling on their perceived change in reported postoperative chest pain.

\section{Methods:}

In this study, we surveyed electrophysiologists using active cooling for greater than six months.

Each operator was asked:

Have you noticed a change in reported postoperative chest pain?

If so, has it increased or decreased?

Estimate the percentage change.

Results: A total of 16 electrophysiologists participated in the survey. Of the 16 electrophysiologists, 14 reported a reduction in postoperative pain. Two electrophysiologists reported no change in postoperative chest pain, and zero reported an increase in postoperative chest pain. The average reduction in physician estimated patient-notifications for chest pain after AF ablation since esophageal cooling was employed was $58 \%$.

Conclusions: The use of active cooling during left atrial ablation for atrial fibrillation is associated with a significant reduction in the number of physician-reported patient calls for postoperative chest pain.

\section{AFS2022-47 Submission Number: 1173634}

Abstract Title: Active Esophageal Cooling During the CONVERGENT Procedure for the Treatment of Long-Standing Persistent Atrial Fibrillation 
Author(s): Ankur Srivastava, MD

Co-Author(s):

Christopher Joseph, BA - University of Texas Southwestern Medical School

Erik Kulstad, MD

Hemal Nayak, MD

University of Chicago

5841 S Maryland Ave, Chicago, IL 60637

Introduction | Objectives: The CONVERGENT procedure is a hybrid operation consisting of epicardial ablation with subsequent endocardial ablation for patients with persistent atrial fibrillation (AF). Despite direct visualization during ablation as well as the use of luminal esophageal temperature (LET) monitoring, serious esophageal injury has been reported. Active esophageal cooling during cardiac ablation has been associated with a reduction in esophageal lesion incidence and severity, with no atrioesophageal fistulas yet reported despite thousands of uses.

Methods: We describe the first recorded uses of active cooling during the CONVERGENT procedure for esophageal protection.

\section{Results:}

Case 1: A 73-year-old male with 7 years of long-standing persistent AF despite multiple trials of antiarrhythmic therapy and cardioversion was referred for the CONVERGENT procedure. The procedure was performed under general anesthesia, and an active esophageal cooling device (ensoETM, Attune Medical, Chicago, IL) was placed and cooled to a temperature of $4^{\circ} \mathrm{C}$. A subxiphoid approach was utilized to visualize the epicardial surface of the posterior left atrium (LA), and a total of 19 lesions were delivered utilizing $30 \mathrm{~W}$ for 90 seconds. As LET is not required when using active cooling, there was no premature discontinuation of energy delivery. Procedurally, the presence of the active cooling device was only evident during two occasions as an indentation in the posterior wall and did not impede ablation.

Case 2: A 68 year old man with drug-refractory long-standing persistent AF and tachycardia-mediated cardiomyopathy was referred for the CONVERGENT procedure. The procedure was performed similarly, with a total of 21 ablation lesions delivered on LA.

\section{Conclusions:}

The cases described here suggest that active cooling does not disrupt the flow of the CONVERGENT procedure but may simultaneously add the benefit of thermal protection. The additional movement required to maneuver the ablation catheter around (anterior) to the esophagus during one lesion did not deter procedure progression. Utilizing active cooling during the CONVERGENT procedure appears to be a simple approach to minimize potential thermal injury in patients with long-standing persistent AF.

AFS2022-48Submission Number: 1174292

Abstract Title: 3 Years Follow-up of LAA Closure in Patients with LAA Thrombus

Author(s): Cem Coteli, MD

\section{Co-Author(s):}

Levent Sahiner, Prof. - Hacettepe University Faculty of Medicine

Baris Kaya, Prof. - Hacettepe University Faculty of Medicine

Ahmet Ates, Asoc. Prof. - Hacettepe University Faculty of Medicine

Hikmet Yorgun, Prof. - Hacettepe University Faculty of Medicine 
Kudret Aytemir, Prof. - Hacettepe University Faculty of Medicine

Hacettepe University Faculty of Medicine, Department of Cardiology

Hacettepe University Department of Cardiology Sihhıye/Ankara

\section{Introduction | Objectives:}

Recent guidelines suggest LAA closure for patients with atrial fibrillation and high bleeding risk or who have oral anticoagulation contraindications. On the other hand, other possible indications for LAA closure are being discussed in the consensus documents, and one possible indication for LAA closure is malign LAA thrombus. LAA thrombus is a challenging factor for both medical treatment and LAA closure.

In this study, our objective was to evaluate long-term results of LAA closure in patients with LAA thrombus.

\section{Methods:}

We presented the single-center experience with 12 months median (6-24 months) follow-up in our previous study entitled "Left Atrial Appendage Occlusion in Patients with Thrombus in Left Atrial Appendage". The same cohort was followed during a median of 36 months (30-48 months). Major adverse clinical events including disabling stroke, clinically relevant hemorrhage, myocardial infarction, and all-cause mortality were recorded.

\section{Results:}

Twelve patients were enrolled in the study. The median age of our study group was 71.8 years (62-83 years). The mean CHA2DS2-VASc score was 4.9 (range, 2-8), and the mean HAS-BLED score was 4.8 (range, 3-6). Amplatzer Amulet LAA closure device was used in all patients. We observed non-cardiovascular mortality in 2 patients during follow-up. Both patients died due to COVID-19 related respiratory failure. In other patients, no clinically significant cerebrovascular events or major adverse cardiac events were observed. Seven patients used ASA plus clopidogrel, 3 patients used direct oral anticoagulant plus clopidogrel as dual antiplatelet therapy for 6 months, and all of them used only single antiplatelet therapy after 6 months. Oral anticoagulant therapy was continued as single antiplatelet therapy in patients without oral anticoagulant contraindication, and all other patients were used ASA after the first six months. No peridevice leak was observed with transesophageal echocardiography at the one-year visit. Routine TEE imaging was not performed after the first twelve months. Because no finding was observed in routine transthoracic echocardiography imaging in any patients.

\section{Conclusions:}

Our study showed that LAA closure is a feasible and safe procedure in patients with LAA thrombus.

\section{AFS2022-48}

Uploaded File(s)

3 Years Follow-up of LAA Closure in Patients with LAA Thrombus 


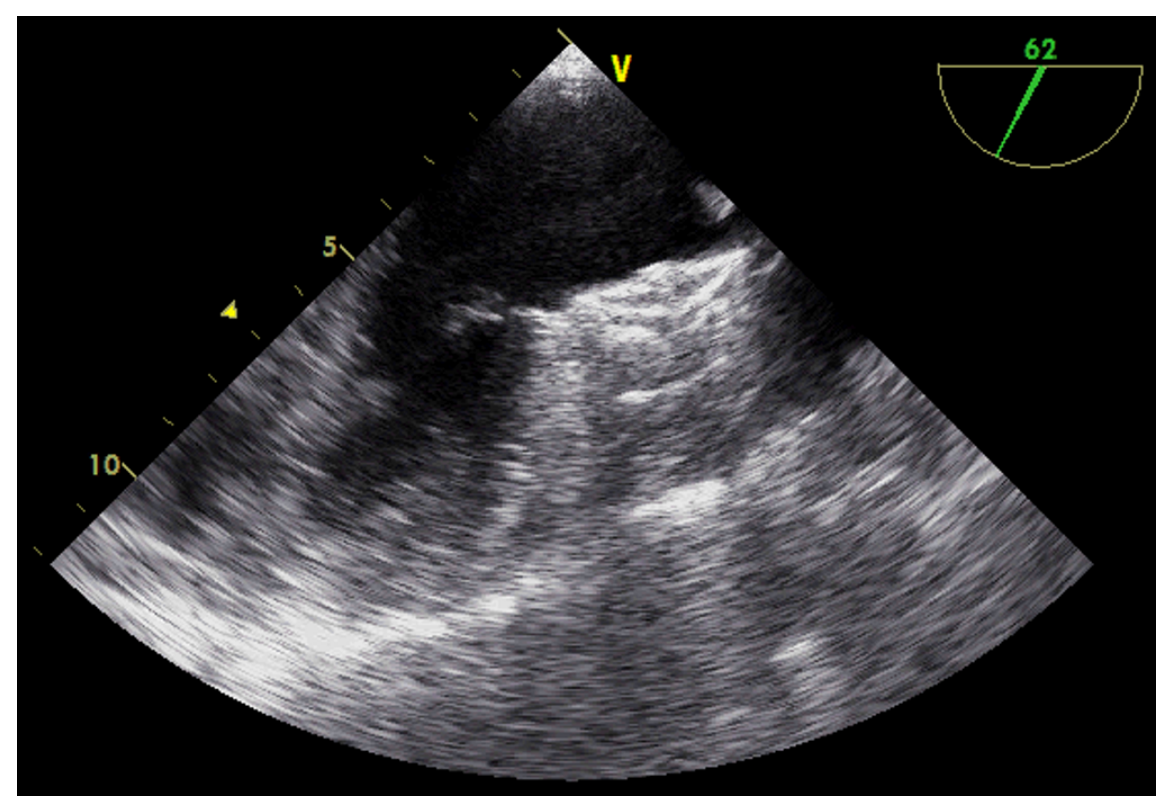

\section{AFS2022-49Submission Number: 1174318}

Abstract Title: Improved left atrial appendage occlusion procedural efficiency using radiofrequency transseptal wire system

Author(s): Cameron Whitler, DO

Co-Author(s):

Brittni McClellan, MD

Harshil Patel, MD

Dhruv Rajpurohit, DO

Frank Kalaba, MD

Herman Kado, MD, FACC

Ashok Kondur, MD, MBA, FACC, FSCAI

Shukri David, MD, FACC

Dipak Shah, MD, FACC, FHRS

Ascension Providence Hospital

16001 W Nine Mile Rd, Southfield, MI 48075

\section{Introduction | Objectives:}

Multiple wire and sheath exchanges result in longer procedure times potentially leading to increased radiation exposure and complications. This study investigates the use of the VersaCross radiofrequency (VCRF) transseptal wire system (Baylis) to improve overall efficiency of left atrial appendage closure (LAAC) compared to the standard RF needle-based workflow.

\section{Methods:}

LAAC using the WATCHMAN FLX device (Boston Scientific) was performed using a needle-based workflow (NRG RF needle, Baylis; 8.5F SL1 sheath, Abbott; ProTrack pigtail wire, Baylis) or VCRF system (RF 
pigtail wire and dedicated 8.5F sheath) under fluoroscopy and TEE guidance. Cases in the needle group were performed as per usual protocol. In the VCRF group, the VCRF pigtail wire was used for vascular access, transseptal puncture (TSP) and for exchanging the WATCHMAN sheath into the LA. Cases were performed consecutively and procedural characteristics were retrospectively compared between the two groups. The primary endpoint was procedural efficiency determined by time to final implant release. Secondary endpoints were time to TSP puncture, LAAC success, fluoroscopy use and procedural complications.

\section{Results:}

A total of 84 patients underwent LAAC using the VCRF system (54) or standard workflow (30). Both groups had similar baseline characteristics. All procedures were successful, with no intraprocedural complications. TSP time was similar between VCRF and standard RF needle-based workflow $(6.6 \pm 2.3$ min vs. $7.4 \pm$ 2.5 min, respectively; $\mathrm{p}=0.16)$. Time to final implant release was $3.9 \mathrm{~min}(13 \%)$ faster in the VCRF group $(\mathrm{p}=0.03$, Fig 1A). There was no difference in overall fluoroscopy time between VCRF and the needle workflow $(7.7 \pm 2.8 \mathrm{~min}$ vs. $9.0 \pm 4.3 \mathrm{~min}$, respectively; $\mathrm{p}=0.11)$; however, fluoroscopy dose was $67 \%$ lower with VCRF $(\mathrm{p}=0.006$, Fig 1B).

\section{Conclusions:}

TSP using a RF wire is similar to a RF needle, but overall procedural efficiency is improved using the RF wire-based system. VCRF decreased the time to implant release by reducing the number of exchanges in the LA and enabling more optimized sheath delivery into the LAA. Better visualization of this system likely leads to a reduced fluoroscopy dose.

\section{AFS2022-49}

\section{Uploaded File(s)}

Figure 1

A

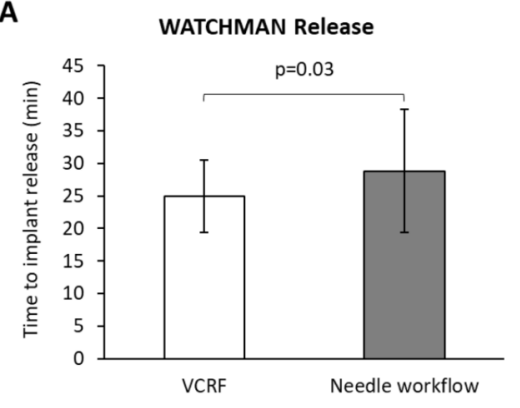

Figure 1. (A) 3.9min faster LAAC and (B) $68 \%$ lower fluoroscopy dose using the VersaCross RF wire-based system (VCRF) compared to the standard needle workflow ( $R F$ needle, $8.5 \mathrm{~F}$ sheath, pigtail exchange wire).

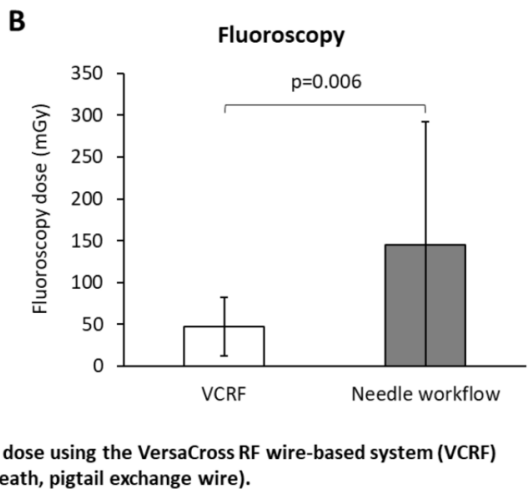

AFS2022-50Submission Number: 1174357

Abstract Title: Ablation of Electrical Activity in the "Blind Spot" of the Lasso Controlled PVI

Author(s): Laura Stix, Dr. med. univ.

Co-Author(s):

Guenter Stix, Ass. Prof. Dr.

Nadir Saoudi, MD, FHRS, FESC, FACC, FEHRA

Marianne Gwechenberger, A.o. Univ.-Prof.in Mag.a Dr.in 
Christian Loewe, Ao. Univ.-Prof. Dr.

Dietrich Beitzke, Dr. med. univ.

Elvis Tumnitz, cand. med.

General Hospital of Vienna

Waehringer Guertel 18-20 1090 Vienna

\section{Introduction | Objectives:}

Continuous lines around the pulmonary veins (PVs) are required for pulmonary vein isolation (PVI), this being the goal at any level of ablation from the PV-ostium away to the antrum. The muscular sleeves are very thin in the PVs and increase substantially in thickness and complexity in the antrum.

Therefore, we suppose that the disappearance of electrical potentials from the lasso-catheter in the PVostium is an insufficiently reliable tool for verifying a complete ablation line at a certain distance away in the antrum.

\section{Methods:}

Lasso-controlled PVI was verified by 1) exit-block, 2) entry-block, 3) negative adenosine test and 4) unexcitability along the ablation line. Immediately after, the area between the position of the lasso in the PV-ostium and the ablation line in the antrum was controlled using ultra-high-density mapping (UHDM).

\section{Results:}

Lasso-controlled PVI was achieved in 50 consecutive patients, verified by all 4 tests. UHDM revealed isolated PVs (100\%), but residual electrical activity between the lasso position and the ablation line was found in the majority of those patients. In 35 patients (70\%) the first UHD-map after the initial PVI found a conduction path through the ablation line. Likewise the right carina $(\mathrm{CaR})$ showed the most gaps, 21 patients $(60 \%$ of those 35$)$. Nine (25\%) patients had a gap in the RSPV, 4 patients $(11 \%)$ in the RIPV. Furthermore in 6 patients $(17 \%)$ we found a gap in the LSPV, 7 patients $(20 \%)$ showed a gap in the LIPV and 7 patients $(20 \%)$ in the $\mathrm{CaL}$

After 12 months $87 \%$ of the paroxysmal AF patients, $50 \%$ of persistent AF patients and $63 \%$ of longlasting persistent AF patients were still in sinusrhythm.

\section{Conclusions:}

UHDM is able to trace electrical potentials into a "blind spot" of lasso-controlled PVI and appears acutely superior to test for complete electrical isolation of an ipsilateral PV antral ablation line.

AFS2022-51Submission Number: 1174368

Abstract Title: MR assessment of AF cryoballoon ablation- induced circumferential PV fibrosis and its correlation with clinical AF recurrence

Author(s): Moshe Rav Acha, MD PhD

Co-Author(s):

Oholi tOVIA-BRODIE, MD

Yoav Michowitz, MD

Firas Bayya, MD

Michael Glikson, MD

Arik Wolak, MD 
share zedek hospital

Shmuel Beit 12 jerusalem israel

Introduction | Objectives:

Use of late Gadolinium Enhancement (LGE) MR to assess baseline atrial fibrosis and predict AF ablation success is under consensus. Nevertheless, MR ability to assess post-ablation lesions and PV 'gaps' is still a major controversy. Some centers report good correlation of PV 'gaps' with redo mapping, while others show poor correlation. Furthermore, complete circumferential PV fibrosis was shown in only $7 \%$ of patients by MR performed just 3 months post successful ablation. Notably, radio-frequency (RF) was the dominant ablation technique in these studies. Our study objective was to assess cryoballoon AF ablation-induced PV fibrosis via post ablation MR and its correlation with 1-year AF recurrence.

\section{Methods:}

Pre and post-ablation MR-LGE to assess baseline and ablation-induced fibrosis, respectively. AF recurrence monitored via multiple ECG, 24-h Holters, and month monitoring after 6 \& 12 months post ablation. MR Analysis performed visually and by ADAS software.

\section{Results:}

17 patients (69 PV) included, age $56 \pm 11$, with paroxysmal AF in $16 / 17$ patients and persistent $\mathrm{AF}$ in 1/17. All had normal LV function, no significant valvular abnormalities, and LA diameter of $45 \pm 6 \mathrm{~mm}$ on TTE. Baseline MR showed minimal LA fibrosis. All patients underwent cryoballoon ablation with successful electrical isolation of all PV. Post ablation MR, performed 3-6 months from ablation, revealed complete circumferential PV fibrosis among 56/69 (81\%) PV's in 10/17 (59\%) of patients. Partial gaps were noticed in 12/69 (17.5\%) PV's among 6/17 (35\%) patients; and 1 patient with absent ablation-induced fibrosis in 1 PV. AF recurred in 4/17 (23.5\%) patients within 1 year, all with partial PV 'gaps' on post ablation MR. Correlation with redo ablation mapping reduced dramatically when post-ablation MR done $>4$ months from ablation.

\section{Conclusions:}

In this proof of concept study, cryoballoon AF ablation resulted in complete circumferential PV fibrosis in $60 \%$ of patients and $80 \%$ of isolated PV, assessed by post-ablation MR. This is in contrast with small minority of circumferential PV fibrosis reported after RF ablations. Good correlation was found between post-ablation PV 'gaps' on MR and AF recurrence, suggesting MRI ability to predict AF recurrence.

\section{AFS2022-52Submission Number: 1174407}

Abstract Title: Accuracy of an automated, real-time algorithm to localize the focal origin of premature atrial contractions and non-PV triggers using non-contact mapping

Author(s): Douglas Gibson, MD

\section{Co-Author(s):}

Pratik Shah, PhD

Derrick Chou, $\mathrm{PhD}$

Vivek Reddy, MD

Scripps Clinic and Prebys Cardiovascular Institute

9888 Genesee Ave, San Diego, CA 92037

Introduction | Objectives: 
Non-pulmonary vein (PV) focal triggers can be identified in 10-33\% of patients referred for catheter ablation of AF. Localization of non-PV AF triggers can be challenging, typically involving recognition of specific intra-atrial activation patterns on multipolar catheters placed in the RA, LA, and CS, together with the surface ECG and a roving multielectrode catheter to help regionalize the area(s) of interest. This conventional method of mapping non-PV triggers is time-consuming and cumbersome. Chamber-wide noncontact mapping may offer a rapid accurate alternative.

Objective : To evaluate the accuracy of an automated, non-contact method to detect and localize PACs and non-PV triggers in real-time.

\section{Methods:}

Five persistent AF patients undergoing AF ablation were screened for non-PV triggers, including administration of isoproterenol, while recording using the noncontact mapping system and non-contact mapping catheter (AcQMap; Acutus Medical Inc.) in the LA or RA (6 chambers evaluated). Recorded data was retrospectively processed using an automated, real-time algorithm (see algorithmic steps in Figure).

The origin site of the most-prevalent PACs in each chamber were confirmed via targeted RF ablation and cessation of PACs from treated site. In one subject, the PAC origin was confirmed via local catheter measurement but was not treated.

Sensitivity and specificity of automated PAC/non-PV trigger detection were evaluated against manual labeling by a human operator using reference EGMs. Computed origin site of each PAC was compared to clinically confirmed/targeted regions.

\section{Results:}

The site of origin was correctly identified in 6 of 6 chambers, corresponding to the clinically diagnosed location in the chamber. The mean sensitivity and specificity of automated PAC/non-PV trigger detection was 0.95 and 0.92 , respectively (see example in Figure).

\section{Conclusions:}

The automated, real-time, non-contact algorithm accurately detected and localized the site of origin of PACs and non-PV triggers.

\section{AFS2022-52}

\section{Uploaded File(s)}




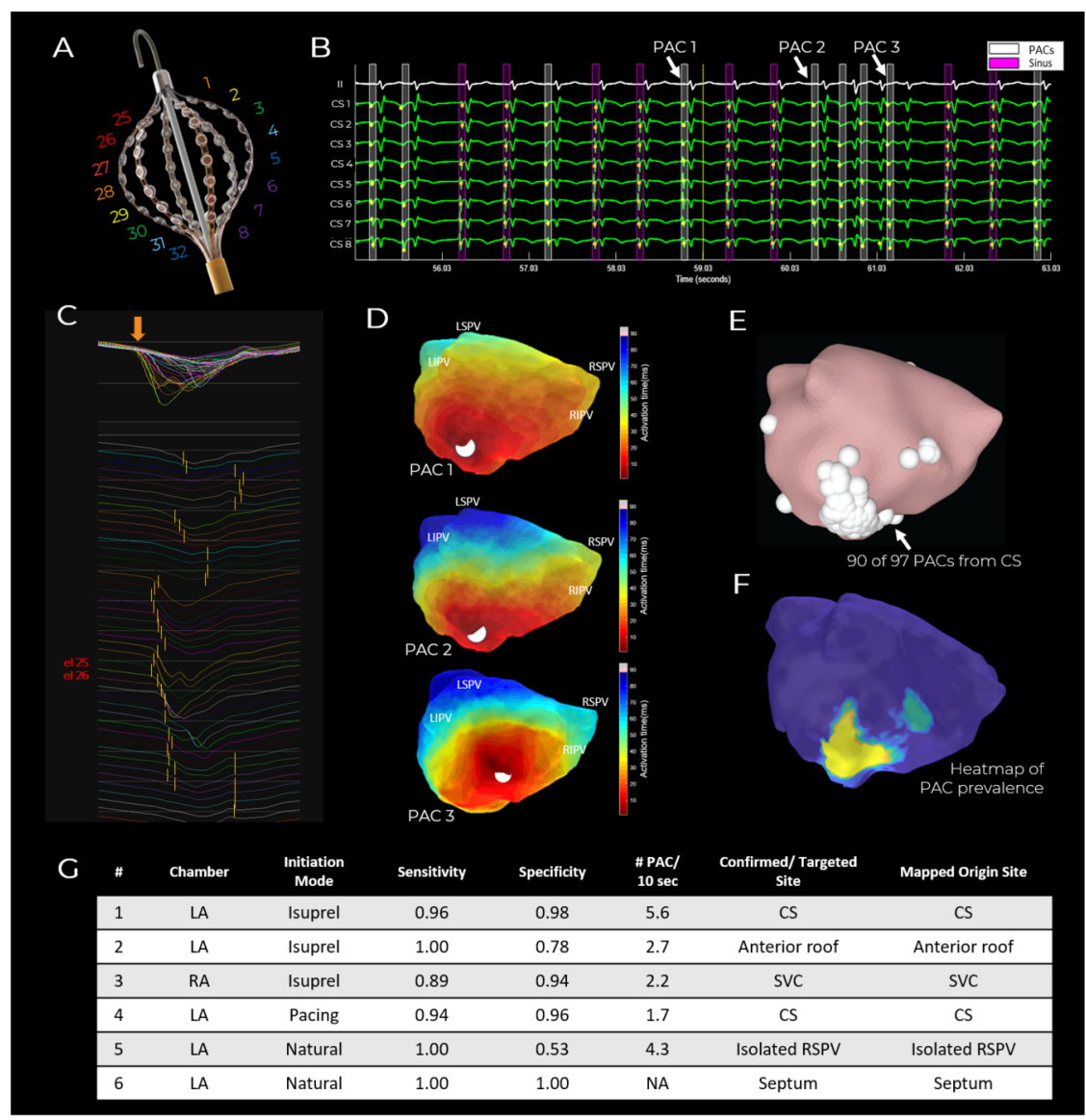

Figure 1. $(A, C)$ Unipolar intracavitary EGMs from the AcQMap catheter are annotated (-dV/dt; orange bars). Representation of annotated timing on numbered electrodes of the mapping catheter that are projected to shell. (B) Reference EGMs with automatically detected PACs (white) and SR (pink). (D) Examples of automatically mapped PACs with LAT map \& marked estimated origin site. (E) Spatial distribution of 97 automatically mapped origin sites in Case \#1. (F) Heatmap of PAC prevalence highlighting CS. (G) Summary results table, including method of PAC initiation, sensitivity/specificity of PAC detection, clinically confirmed or targeted site, and automatically mapped origin site of highest PAC prevalence.

\section{AFS2022-53Submission Number: 1174456}

Abstract Title: A Rare Cause of Atrial Fibrillation

Author(s): Ankita Dubey, MD, MS

Co-Author(s):

Lynna Alnimer, MD

Hawra Kamal, MD

Rizvi Humaira, MD

Souheil Saba, MD

Ascension Providence Hospital

16001 W Nine Mile Rd, Southfield, MI 48075

Introduction | Objectives: Apical hypertrophic cardiomyopathy (ApHCM) is defined as left ventricular hypertrophy predominantly involving the apex with a non-obstructive physiology. First described in 1976 by Sakamoto et al, 15-25\% of Asian patients with HCM have ApHCM, but the prevalence in non-Asian 
population remains low at 1-3\%. The inheritance is autosomal dominant, however, sporadic forms of acquired mutations in the sarcomere protein gene exists. Symptoms are more common in the fourth decade of life with male predominance. Most patients remain asymptomatic while others can develop arrhythmias, angina or myocardial infarction.

Methods: We report a case of a 51-year-old African American male with a medical history of hypertension who presented to the hospital due to palpitations. Upon presentation, the patient was afebrile, blood pressure was $138 / 88 \mathrm{mmHg}$, heart rate was 150 beats per minute (bpm), respiratory rate was 20 and oxygen saturation was $98 \%$ on room air. Physical examination revealed an irregularly irregular rhythm.

Results: High sensitivity cardiac troponin T and TSH level were within normal limits. Electrocardiogram (EKG) showed atrial fibrillation with rapid ventricular rate of $143 \mathrm{bpm}$, left ventricular hypertrophy and deep $\mathrm{T}$ wave inversions in the left precordial leads (Figure 1a). Transthoracic echocardiogram (TTE) was completed to evaluate for potential valvular or other structural heart disease, and it demonstrated marked hypertrophy of the left apical ventricular apex along with spade-like configuration of the left ventricular cavity at the end of diastole (Figure 1b). The echocardiographic finding of spade-like configuration of the left ventricular cavity along with the deep $\mathrm{T}$ wave inversions noticed on the EKG were characteristic of ApHCM also known as Yamaguchi syndrome. After management of the acute episode with diltiazem infusion, the patient was discharged two days later on metoprolol for rate control and apixaban for thromboembolic risk reduction.

Conclusions: Recognizing and diagnosing this disease is key as recent data suggests that ApHCM carries a high mortality risk, approaching that of classic HCM. Our case also highlights that Yamaguchi syndrome must be considered in the differential diagnosis, when evaluating patients with $\mathrm{T}$ wave changes that may be mistaken as ischemia.

\section{AFS2022-53 Uploaded File(s)}

\section{A Rare Cause of Atrial Fibrillation}



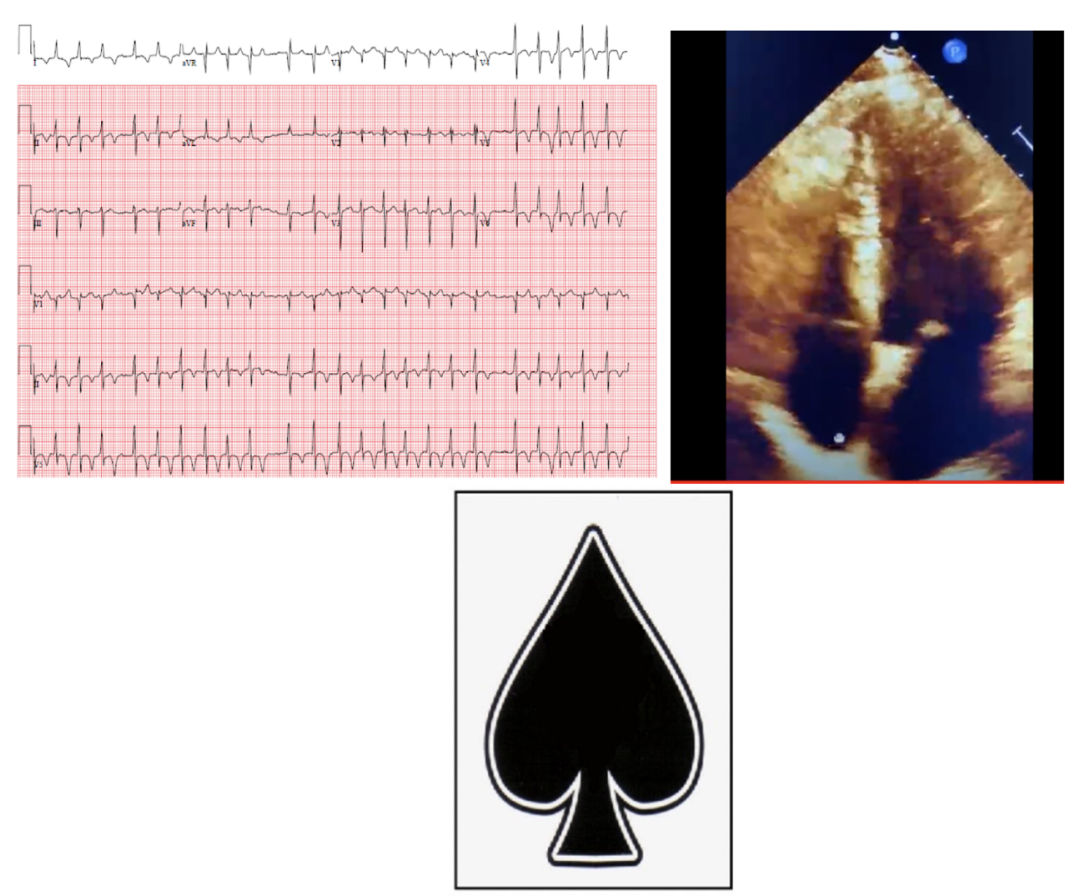

\begin{abstract}
Figure 1: (A) Electrocardiogram on admission showed deep T wave inversions in precordial leads (V4-V6). (B) Four-chamber view on transthoracic echocardiogram showing spade-like configuration of left ventricular cavity at end diastole. (C) Pictorial representation of Ace of spades
\end{abstract}

\title{
AFS2022-54Submission Number: 1174460
}

Abstract Title: DOLLAR Score System to Predict Recurrence for Sustained Persistent Atrial Fibrillation After Ablation: A Pilot Study

Author(s): Devansh H. Patel, MD

\section{Co-Author(s):}

Stephen Mester, MD, FACC - Bay Area Cardiology

Umesh Gowda, MD, FACC - Bay Area Cardiology

Tehreen Khan, MD, FACC - Bay Area Cardiology

Ranchhod Khant, MD, FACC

Juna Misiri, MD, FACC

Christian Perzanowski, MD, FACC, FHRS, FAHA

HCA Healthcare/USF Morsani College of Medicine GME: Brandon Regional Hospital

119 Oakfield Dr, Brandon, FL 33511

Introduction | Objectives: Radiofrequency ablation (RFA) is a widely accepted treatment for sustained persistent atrial fibrillation (SPAF). Predicting the risk of sustained recurrence may help to identify patients who are unlikely to respond to RFA. We hypothesize a new scoring system to indicate recurrent SPAF, post RFA. 
Methods: A retrospective study evaluating 97 patients with SPAF followed post-ablation was conducted. Six patients were lost to follow-up. The variables analyzed included: (D) diabetes mellitus, (O) obstructive sleep apnea, (L) left atrial diameter/body surface area [?]2.3, (L) left atrial diameter*100/body mass index $[?] 14,(\mathrm{~A})$ age [?]75 years, and (R) regurgitation of the mitral valve (moderate or severe). A maximum score was 6 , with a point assigned to each parameter. All patients underwent wide area circumferential ablation or linear ablation at the operator's discretion with an irrigated-tip catheter, and electroanatomic mapping (CARTO) assessed the degree of atrial fibrosis.

Results: This sample consisted of $59.7 \%$ males, with a mean age of $69.7 \pm 9.8$ years, and $27.8 \%$ had prior AF ablation. Mean follow-up $779 \pm 434.8$ days. The mean DOLLAR score of 2.15 and CHADS2VASc score of 2.73. Ultimately, $28.5 \%$ (CI 95\% 19.5,39) of patients had recurrent SPAF and required further management. The DOLLAR score of [?]3 and CHADS2VASc of [?]3 was compared (Figure 1a). There was no significant difference for a DOLLAR score of [?]3 to predict recurrence of AF compared to CHADS2VASc [?]3 ( $\mathrm{P}=0.706)$. The analysis would suggest equivalence to predict recurrent SPAF. A significant difference in the distribution of LA diameter $(\mathrm{cm})$ was found between patients with DOLLAR scores [?]3 and $<3$ (Figure1b).

Conclusions: The DOLLAR score [?]3 score was non-inferior to CHADS2VASc [?] 3 in predicting recurrent SPAF after RFA. More studies are needed to more accurately identify optimal candidates who are likely to derive benefit from RFA for SPAF and exclude those who are not.

\section{AFS2022-54 Uploaded File(s)}

Image showing comparison of survival curves and left atrial size based on DOLLAR score

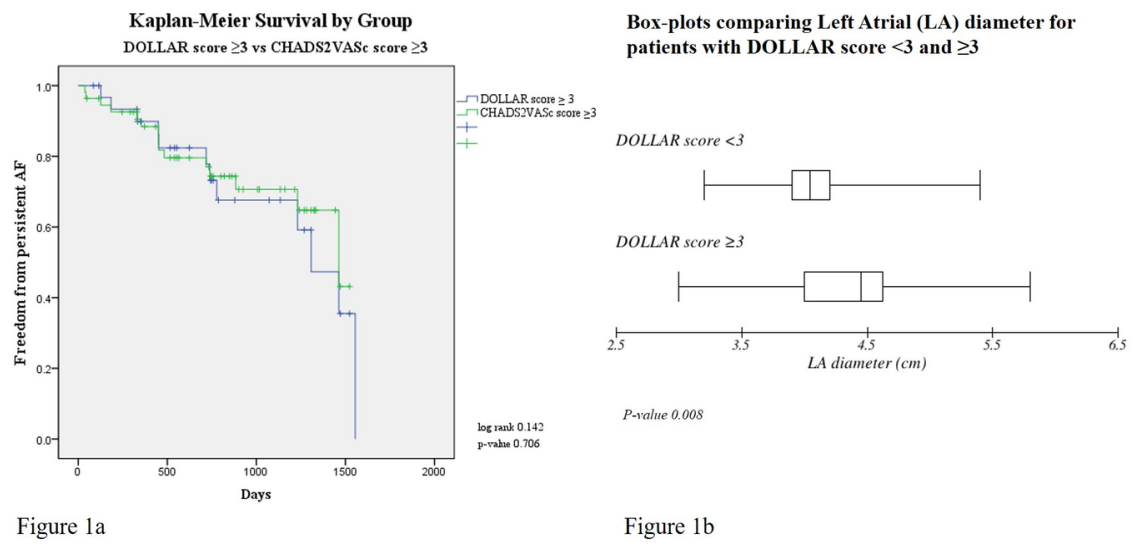

\section{AFS2022-55Submission Number: 1174461}

Abstract Title: Hyperthyroidism Manifesting as Atrial Fibrillation Subsequent to COVID-19 Vaccination

Author(s): Devansh H. Patel, MD

Co-Author(s):

Juna Misiri, MD, FACC

Umesh Gowda, MD, FACC - Bay Area Cardiology

Tehreen Khan, MD, FACC - Bay Area Cardiology

Ranchhod Khant, MD, FACC

Stephen Mester, MD, FACC - HCA Healthcare/USF Morsani College of Medicine GME: Brandon Regional Hospital 
Christian Perzanowski, MD, FACC, FHRS, FAHA

HCA Healthcare/USF Morsani College of Medicine GME: Brandon Regional Hospital

119 Oakfield Dr, Brandon, FL 33511

\section{Introduction | Objectives:}

COVID-19 vaccination campaigns are underway. Rarely, autoimmune reactions directed towards glandular structures have been identified. Vera-Lastra and colleagues, et al.,2021 previously reported clinical hyperthyroidism following vaccination. Our group has observed clinical hyperthyroidism following COVID-19 vaccination. The true incidence is unknown and may be underreported. We present a case of atrial fibrillation due to an autoimmune thyroid disorder triggered by COVID vaccination.

Methods: N/A

Results: A 44-year-old man with no cardiovascular history presented with sudden onset tachycardic events for a few months subsequent to Pfizer COVID-19 Vaccination. The patient's personal external wearable monitoring device revealed an elevated heart rate (160s to 170 beats/min) intermittently. Holter monitoring revealed paroxysmal atrial fibrillation (Figure 1). An autoimmune response was suspected against the thyroid gland, confirmed by laboratory results (Table 1). Echocardiogram showed an ejection fraction of $65 \%$ with borderline left ventricular hypertrophy and mild left atrial dilation; negative stress test. The patient was diagnosed with hyperthyroidism and was treated with Methimazole and Beta-1 selective blocker.

\section{Conclusions:}

Exposure to the COVID-19 vaccine may trigger an autoimmune response against the thyroid gland leading to atrial fibrillation. This is a rare complication; clinicians should be aware of this adverse effect. The natural history of hyperthyroidism post-COVID-19 vaccine remains to be elucidated by further studies.

\section{AFS2022-55 Uploaded File(s)}

\section{Holter monitoring revealing Atrial Fibrillation rhythm and Thyroid Studies}

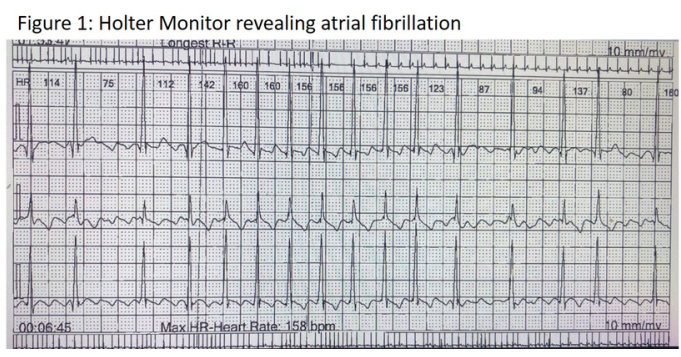

\begin{tabular}{|c|c|c|}
\hline Laboratory tests & Results (unit) & $\begin{array}{l}\text { Normal range } \\
\text { (unit) }\end{array}$ \\
\hline TSH & $<0.01(\mathrm{mlU} / \mathrm{L})$ & $0.5-5.0(\mathrm{~m} / \mathrm{U} / \mathrm{L})$ \\
\hline Total T4 & 11.1 (mcg/dL) & $4.9-10.8(\mathrm{mcg} / \mathrm{dL})$ \\
\hline Free $\mathrm{T} 3$ & $6.2(\mathrm{pmol} / \mathrm{L})$ & 2-6 (pmol/L) \\
\hline $\begin{array}{l}\text { Anti thyroglobulin } \\
\text { peroxidase }\end{array}$ & $413(\mathrm{IU} / \mathrm{ml})$ & $<9(\mathrm{IU} / \mathrm{ml})$ \\
\hline $\begin{array}{l}\text { Thyroid-stimulating } \\
\text { immunoglobulin }\end{array}$ & $308(\%)$ & $<140(\%)$ \\
\hline
\end{tabular}

AFS2022-56Submission Number: 1174499

Abstract Title: Successful Insertion of Left Atrial Appendage Occlusion Device using Intracardiac Echocardiography and CT Imaging in a Patient with an Unsuccessful Left Atrial Ligation Surgery

Author(s): Devansh H. Patel, MD

Co-Author(s):

Robert Dewhurst, MD, FACC

Umesh Gowda, MD, FACC - Bay Area Cardiology

Tehreen Khan, MD, FACC - Bay Area Cardiology

Stephen Mester, MD, FACC - Bay Area Cardiology 
Juna Misiri, MD, FACC

Christian Perzanowski, MD, FACC, FHRS, FAHA

HCA Healthcare/USF Morsani College of Medicine GME: Brandon Regional Hospital

119 Oakfield Dr, Brandon, FL 33511

\section{Introduction | Objectives:}

Left atrial appendage closure (LAAC) with the Watchman Flex device is a viable alternative to chronic anticoagulation for preventing atrial fibrillation (AF) induced stroke in patients at risk of hemorrhagic complications, as demonstrated in the PREVAIL and PROTECT-AF trials. Left atrial appendage (LAA) ligation is the surgical treatment for AF. We present a case where surgical ligation was left incomplete, and transesophageal echocardiogram (TEE) did not show the true LAA ostium. Pre-op CT imaging and intracardiac echocardiogram (ICE) could identify the accurate LA morphology for a successful LAAC.

Methods: N/A

Results:

An 81-year-old man with chronic atrial fibrillation presented to the office with recurrent ecchymosis and chronic anemia precipitated by an Anti-Xa anticoagulation agent. He underwent multiple radiofrequency ablations and DC cardioversions and has a remote history of an incomplete Mini-Maze procedure/LAA ligation. TEE showed incomplete LAA ligation but could not accurately delineate the ostium size. CT imaging software (WATCHMAN TruPlan, Boston Scientific, MA, and Circle CVI, Canada) visualized the LA morphology and measured the ostium size as $16.1 \mathrm{~mm}$ with a depth of $15.4 \mathrm{~mm}$. LAA angiogram indicated a multi-lobed chamber. The patient underwent a successful implant of size 20mm Watchman Flex device using ICE. The patient met all the PASS criteria demonstrating final compression of $20 \%$, and the device was located after the circumflex artery and on the level with the inferior margin of the fibrotic waist.

\section{Conclusions:}

In cases of incomplete LAA ligation, ICE guided by Preop TruPlan CT imaging software can lead to successful implantation of the Watchman Flex device.

\section{AFS2022-56}

Uploaded File(s)

Figure 1: (A) Intracardiac 135 degree angle depicting appropriate device position (B) TEE simulation with CT scan showing appendage orifice (C) ICE imaging 45-degree perspective illustrating optimal LAAO location at the level of the circumflex artery (D) 3D image showing LA appendage waist and insertion site (E) 3D image showing intracardiac trajectory and angle of approach (F) Angiogram of the left atrial appendage; arrows denote the location of the narrow fibrotic waist outside the appendage ostium 

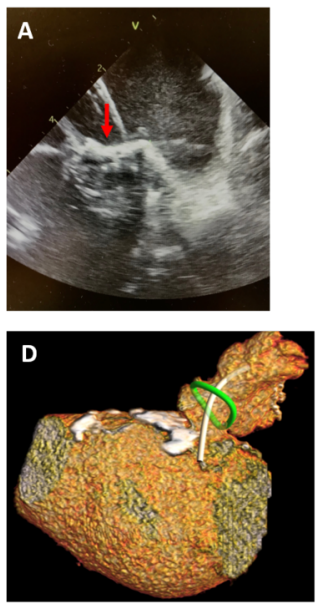
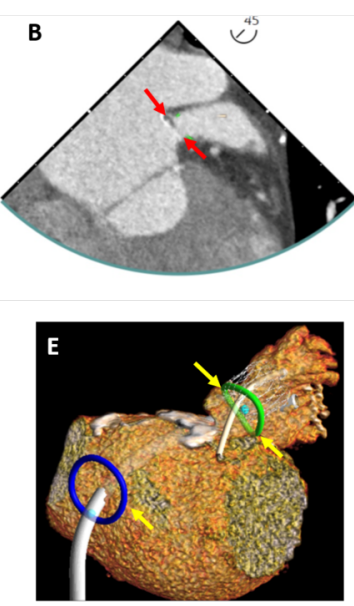
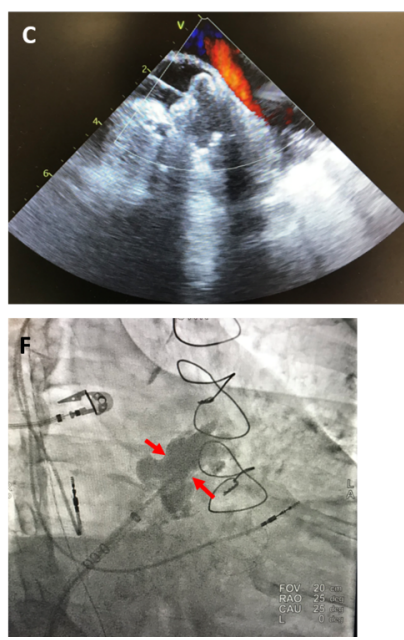

AFS2022-57Submission Number: 1174478

Abstract Title: Evaluating the Learning Curve of a Novel Non-Contact 3D Mapping and Navigation System.

Author(s): Yoel Vivas, MD FHRS

Co-Author(s):

Janelle Lambiris, BSN RN

The Arrhythmia Center of South Florida

5258 Linton Blvd ste 106 Delray Beach FL 33484

Introduction | Objectives: Background: 3D contact mapping is currently the standard of care for electrophysiology procedures when cardiac ablation for atrial or ventricular arrhythmiastreatment are necessary. A novel noncontact charge density mapping system (AcQMap High-Resolution Imaging and Mapping System) has been designed toovercome limitations of existing technologies by allowing electrophysiologist to quickly map and remap as a cardiac procedure progresses with the ultimate goal of sinus rhythm.

\section{Methods:}

Objective: The investigators have set out to evaluate and compare the learning curve with respect to time of electro-anatomical mapping and subsequently comparenoncontact to contact mapping times as a benchmark for improvement. Methods: Retrospective collection and analysis of several mapping measurements were performed from left atrial procedures. Sixty consecutive patients were treated over a 10-month period in a single, high volume ablationcenter with two operators. The initial 10 patient's data were compared to the most recent 10 patients to evaluate the impact of a learning curve on electro-anatomical mapping.

\section{Results:}

There were no significant differences in age or gender respective to the first 10 or most recent 10 subjects treated $(\mathrm{p}>0.05)$. Statistically shorter times were observed in anatomical editing and image creation thus yielding credence that a learning curve does exist in the creation of the ultrasound anatomy with the noncontact technology as observed in the table below $(\mathrm{p}<0.05)$. With the noncontact mapping system there were no significant changes in the electrical recording, processing, or total mapping times. When comparing noncontact and contact electrical mapping, statistically significant differenceswere observed in the initial 10 patients (pprox.. 1 min vs. $14.4 \mathrm{~min}$ ) and the most recent 10 patients (pprox.. 1 min vs. 10.8 min) groups compared $(\mathrm{P}<0.001)$. 
Conclusions: Conclusions: In a retrospective study and independent of physician operators, the noncontact mapping system demonstrated a significant learning curve (faster) in anatomy creation and 9-to-13-minute differential in electrical mapping when compared to conventional mapping technologies. The safety profile for both technologies were similar.

\section{AFS2022-57 Uploaded File(s)}

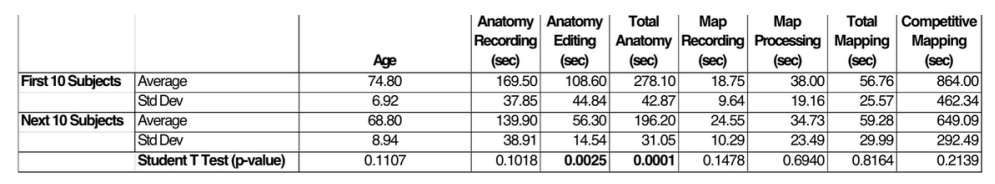

\section{AFS2022-58 Submission Number: 1174489}

Abstract Title: Accuracy of an automated, real-time algorithm to localize the focal origin of clinically simulated premature atrial contractions using non-contact mapping

Author(s): Pratik Shah, $\mathrm{PhD}$

Co-Author(s):

Dan Lagalante, BS

George Thomas, MD

Andrea Natale, MD, F.A.C.C., F.H.R.S., F.E.S.C.

Dhanunjaya Lakkireddy, MD

Acutus Medical Inc

2210 Faraday Ave \#100, Carlsbad, CA 92008

\section{Introduction | Objectives:}

Non-pulmonary vein (PV) triggers can be identified in up to $33 \%$ of patients during catheter ablation of AF. Localization of non-PV AF triggers can be challenging and typically involves recognition of specific intraatrial activation patterns on multipolar catheters placed in the RA, LA, and CS, together with the surface ECG and a roving multielectrode catheter to help regionalize the area(s) of interest. This conventional method of mapping non-PV triggers is time-consuming and very difficult for localizing transient triggers initiating AF. Chamber-wide noncontact mapping offers a rapid, accurate alternative.

Objective : To validate an automated, non-contact method to detect and localize clinically simulated premature atrial contractions (PACs) in real-time.

\section{Methods:}

In 5 patients undergoing ablation for persistent $\mathrm{AF}$ as part of a prospective registry study (DISCOVER-US, NCT04431544), PACs were electrically or mechanically simulated via pacing or catheter manipulation while recording using the non-contact mapping system and mapping catheter (AcQMap, Acutus Medical) in the LA or RA. Recorded data was processed using an automated, real-time algorithm (Figure ).

In each chamber, the region of simulated PAC origin was determined and compared to the known region of simulated initiation. Sensitivity and specificity of the automated PAC detection algorithm were evaluated against manual labeling by a human operator using reference EGMs.

\section{Results:}


The site of origin was correctly identified for $95.1 \%$ of simulated PACs (58 of 61), corresponding to the known initiation site. Three remaining PACs during QRS or T wave were not mappable due to interference of far-field signals. The mean sensitivity and specificity of automated PAC detection using reference EGMs was 0.98 and 0.77 , respectively. Examples of identified PACs and study results table are shown inFigure .

\section{Conclusions:}

The automated, real-time, non-contact algorithm accurately detected and localized the site of origin of paced and mechanically generated PACs. Implementation of strategies to mitigate QRS and T interference will further improve the reliability and accuracy of this approach.

\section{AFS2022-58}

\section{Uploaded File(s)}
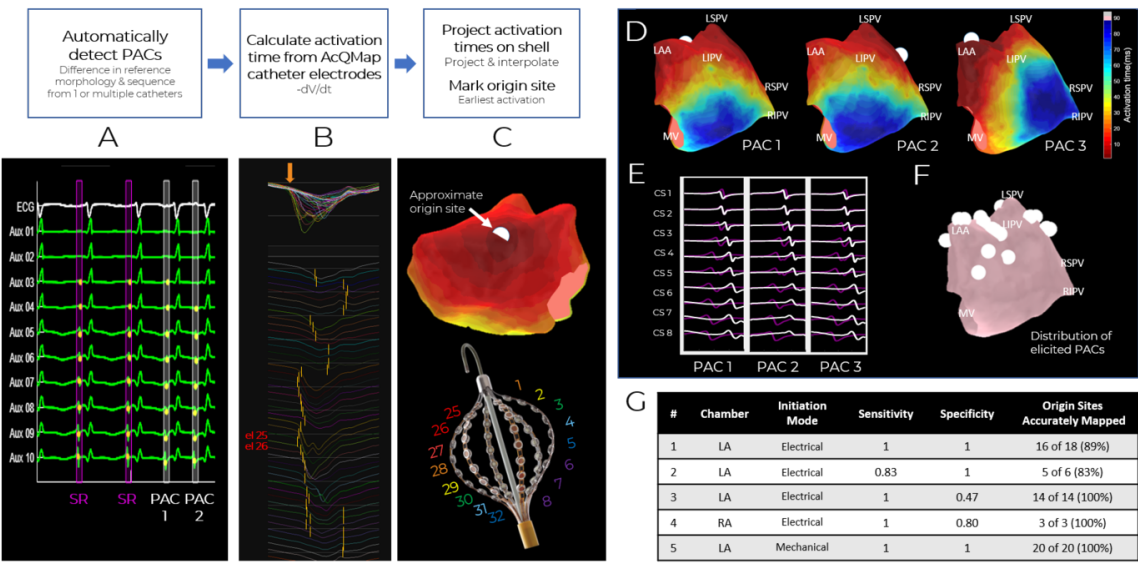

Figure 1. (A-C) Processing steps for Trigger Mapping algorithms. (A) Automatically detect unique beats, shown in white. Colored beats correspond to rhythms with consistent morphology. (B) Unipolar intracavitary ECMs from the ACQMap catheter are annotated (-dV/dt; orange bars). (C) Representation of annotated timing on numbered electrodes of the mapping catheter \& projection to shell. origin site. (D) Examples of automatically mapped PACS with LAT map \& marked estimated origin site (white). (E) Reference ECMs with automatically detected PACS and SR. (F) Spatial distribution of all 16 automatically mapped origin sites in Patient 1. (G) Table of results.

\section{AFS2022-59Submission Number: 1174492}

Abstract Title: Reproducible correlation between non-contact charge density amplitude to bipolar voltage in stable atrial tachycardia

Author(s): Pratik Shah, PhD

\section{Co-Author(s):}

Derrick Chou, $\mathrm{PhD}$

Rui Shi, MD, PhD

Tom Wong, MD, FRCP

Acutus Medical Inc

2210 Faraday Ave \#100, Carlsbad, CA 92008

Introduction | Objectives: Low bipolar voltage (BV) amplitude from contact catheters is broadly used as a metric for compromised tissue substrate and bipolar voltage thresholds have become more objective with frequent use and familiarity. Non-contact (NC) charge density (CD) amplitude in atrial tachycardia (AT) can be collected rapidly but has not been analyzed for correlation to BV amplitude. CD amplitude maps 
may offer higher resolution and less measurement variation (e.g. wavefront or electrode direction) than BV maps. Objective : To compare the spatial consistency of regions of low NC-CD amplitude to regions of low BV amplitude in AT.

\section{Methods:}

Rapid, non-contact CD maps of AT were created with the SuperMap mapping mode of the NC mapping system (AcQMap, Acutus Medical). Peak-to-peak (P-P) CD amplitude was calculated at all locations ( $>3500 \mathrm{CD}$ points) throughout the chamber. Contact BV EGMs (3.5mm spacing) were collected from incidental contact of the mapping catheter. BV EGMs were paired with the corresponding, closest CD EGM. Paired amplitudes were used to calibrate $\mathrm{CD}$ to a $\mathrm{mV}$ equivalent $(\mathrm{mV}$ eq $)$ for each map. Maps were also collected using the HDGrid catheter and Ensite Precision mapping system (Abbott) for qualitative comparison. Conventional scar thresholds (Precision) were used to define regions of scar and low amplitude in $\mathrm{CD} \mathrm{mV}$ eq which were compared to complementary regions in BV maps using AUC analysis.

\section{Results:}

Fourteen ATs (4 focal, 10 re-entrant, $7 \mathrm{LA}$ ) with average cycle length of $291 \pm 53 \mathrm{~ms}$ in 13 patients (age $68.8 \pm 10.1 ; 13$ Male) were studied. Correlation and spatial accuracy of low amplitude zones in BV and CD amplitude maps and the Precision system are shown in Figure 1. Correspondence of CD and BV amplitude (503 \pm 410 points per map) is provided for one representative patient and patient cohort $\left(\mathrm{R}^{2}=0.57\right)$. Mean AUC is $0.89 \pm 0.07$ across all maps.

\section{Conclusions:}

Spatial accuracy of scar and low-amplitude regions in NC-CD amplitude maps are reproducible and wellcorrelated to $\mathrm{BV}$. The $\mathrm{mV}_{\text {eq }}$ calibration of $\mathrm{CD}$ is a reliable correlate to $\mathrm{BV}$.

\section{AFS2022-59}

\section{Uploaded File(s)}
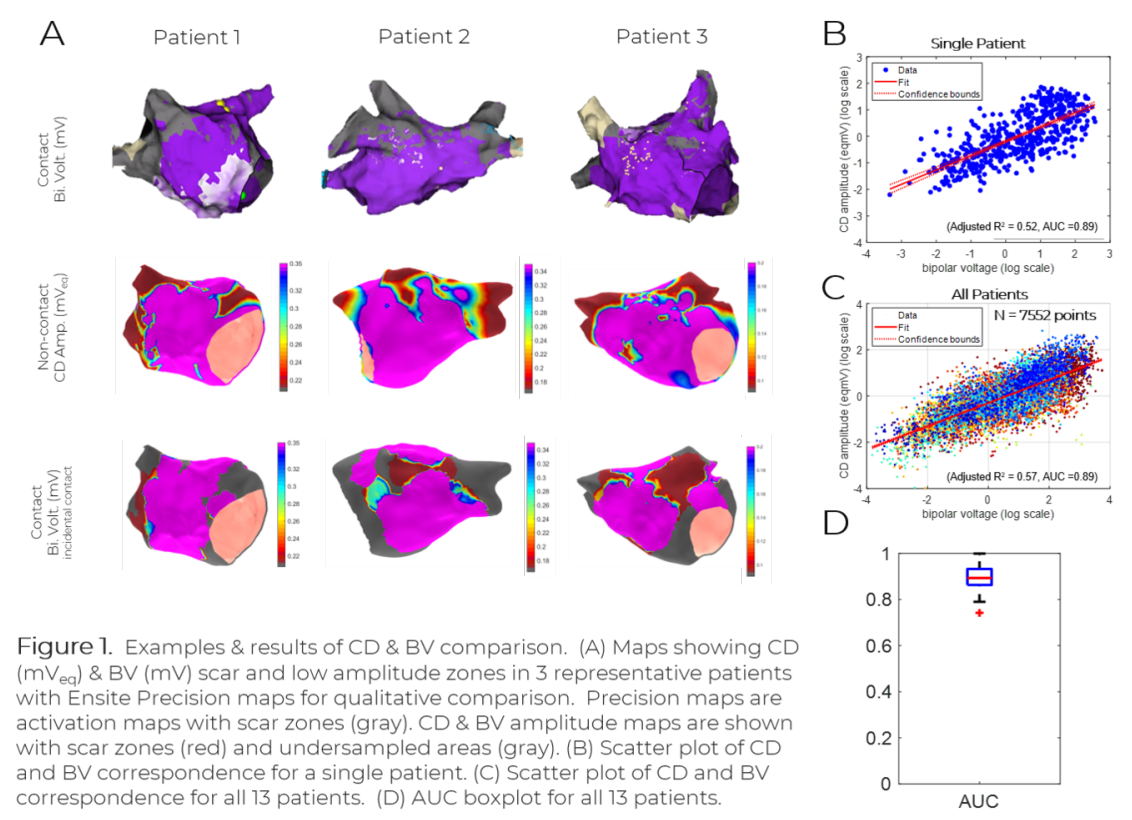